\title{
Convergence Analysis of Iterative Methods for Nonsmooth Convex Optimization over Fixed Point Sets of Quasi-Nonexpansive Mappings
}

\author{
This work was supported by the Japan Society for the Promotion of Science \\ through a Grant-in-Aid for Scientific Research (C) (15K04763).
}

\author{
HIDEAKI IIDUKA \\ Department of Computer Science, Meiji University 1-1-1 Higashimita, \\ Tama-ku, Kawasaki-shi, Kanagawa, 214-8571 Japan. (iiduka@cs.meiji.ac.jp)
}

\begin{abstract}
This paper considers a networked system with a finite number of users and supposes that each user tries to minimize its own private objective function over its own private constraint set. It is assumed that each user's constraint set can be expressed as a fixed point set of a certain quasi-nonexpansive mapping. This enables us to consider the case in which the projection onto the constraint set cannot be computed efficiently. This paper proposes two methods for solving the problem of minimizing the sum of their nondifferentiable, convex objective functions over the intersection of their fixed point sets of quasi-nonexpansive mappings in a real Hilbert space. One method is a parallel subgradient method that can be implemented under the assumption that each user can communicate with other users. The other is an incremental subgradient method that can be implemented under the assumption that each user can communicate with its neighbors. Investigation of the two methods' convergence properties for a constant step size reveals that, with a small constant step size, they approximate a solution to the problem. Consideration of the case in which the step-size sequence is diminishing demonstrates that the sequence generated by each of the two methods strongly converges to the solution to the problem under certain assumptions. Convergence rate analysis of the two methods under certain situations is provided to illustrate the two methods' efficiency. This paper also discusses nonsmooth convex optimization over sublevel sets of convex functions and provides numerical comparisons that demonstrate the effectiveness of the proposed methods.
\end{abstract}

Keywords: fixed point, incremental subgradient method, nonsmooth convex optimization, parallel subgradient method, quasi-nonexpansive mapping

Mathematics Subject Classification: 65K05, 90C25, 90C90

\section{Introduction}

This paper focuses on a networked system consisting of a finite number of participating users and considers the problem of minimizing the sum of their nondifferentiable, convex functions over the intersection of their fixed point constraint sets of quasi-nonexpansive mappings in a real Hilbert space. Optimization problems with a fixed point constraint (see, e.g., 6, 15, 17, 36]) enable consideration of constrained optimization problems in which the 
explicit form of the metric projection onto the constraint set is not always known; i.e., the constraint set is not simple in the sense that the projection cannot be easily calculated.

The motivations for considering this problem are to devise optimization algorithms that have a wider range of application than previous algorithms for convex optimization over fixed point sets of nonexpansive mappings [6, 15, 17, 36] and to solve the problem by using parallel and incremental optimization techniques [3. Chapter 27], 4, Section 8.2], [13, 33, [39, PART II].

Many optimization algorithms have been presented for smooth or nonsmooth optimization. The parallel proximal algorithms [3, Proposition 27.8], [10, Algorithm 10.27], [30] are useful for minimizing the sum of nondifferentiable, convex functions over the whole space. They use the ideas of the Douglas-Rachford algorithm [3, Chapters 25 and 27], 88, 10, 11, 21] and the forward-backward algorithm [3, Chapters 25 and 27], 7, 9, 10, which use the proximity operators [3, Definition 12.23] of nondifferentiable, convex functions. The incremental subgradient method [4, Section 8.2], [5, 13, 18, 19, 25, 33, and projected multi-agent algorithms 22, 26, 27, 28 can minimize the sum of nondifferentiable, convex functions over certain constraint sets by using the subgradients [32, Section 23] of the nondifferentiable, convex functions instead of the proximity operators. The random projection algorithms [24, 35] and the distributed random projection algorithm 20] are useful for constrained convex optimization when the constraint set is not known in advance or the projection onto the whole constraint set cannot be computed efficiently. The incremental subgradient algorithm [13, Sections 3.2 and 3.3] and the asynchronous distributed proximal algorithm [31, Section 6] can work on nonsmooth convex optimization over sublevel sets of convex functions onto which the projections cannot be easily calculated. The incremental and parallel gradient methods [14, 16] and an algorithm to accelerate the search for fixed points [15] can perform smooth convex optimization over the fixed point sets of nonexpansive mappings. There have been no reports, however, on optimization algorithms for nonsmooth convex optimization with fixed point constraints of quasi-nonexpansive mappings.

This paper describes two methods for solving the main problem considered in the paper. One is a parallel subgradient method that can be implemented under the assumption that each user can communicate with other users. The other is an incremental subgradient method that can be implemented under the assumption that each user can communicate with its neighbors. The proposed methods do not use proximity operators, in contrast to conventional asynchronous distributed or parallel proximal algorithms. Moreover, they can optimize over fixed point sets of quasi-nonexpansive mappings, in contrast to conventional incremental subgradient algorithms.

The intellectual contribution of this paper is to enable one to deal with nonsmooth convex optimization over the fixed point sets of quasi-nonexpansive mappings, especially in contrast to recent papers [14, 15] that discussed smooth convex optimization over the fixed point sets of nonexpansive mappings.

To clarify this contribution, let us consider the case where each user in the networked system tries to minimize its own private objective function over a sublevel set of a nonsmooth convex function, where one assumes each user can use the subgradients of the nonsmooth convex function. Although the projection onto the sublevel set cannot be easily computed within a finite number of arithmetic operations, each user can compute the subgradient projection [2, Proposition 2.3], [34, Subchapter 4.3] that satisfies the quasi nonexpansivity condition, not the nonexpansivity condition (see Section 5 for the definition of the subgradient projection). Since the sublevel set coincides with the fixed point set of the subgradient projection, the problem considered in the whole system can be expressed as the problem of minimizing the sum of all users' objective functions over the intersection of the fixed point 
sets of quasi-nonexpansive mappings (see [37] for applications of the problem and the relaxation method for the problem). The proposed methods can thus be applied to nonsmooth convex optimization over sublevel constraint sets of nonsmooth convex functions.

The previously reported algorithms [14, 15] cannot work on nonsmooth convex optimization over sublevel sets of nonsmooth convex functions. This is because they can be applied only when the constraint sets can be represented by fixed point sets of nonexpansive mappings and can work under the restricted situation such that all users' objective functions are smooth and the gradients of their objective functions are Lipschitz continuous and strongly or strictly monotone. The numerical examples section (Section 5) considers a concrete nonsmooth convex problem over the intersection of sublevel sets of nonsmooth convex functions and describes how the proposed methods can solve it.

Another contribution of this paper is analysis of the proposed methods' convergence for different step-size rules. A small constant step size is shown to result in an approximate solution to the main problem. It is also shown that the sequence generated by each proposed method with a diminishing step size strongly converges to the solution to the problem under certain assumptions. In contrast to the convergence analyses of the previously reported algorithms [14, 15, we cannot directly apply smooth convex analysis and fixed point theory for nonexpansive mappings to convergence analysis of the proposed methods. However, this problem is solved by using the subgradients of nonsmooth convex objective functions and by modifying the algorithms presented in [14] to make fixed point theory for quasi-nonexpansive mappings applicable. The rates of convergence of the two methods under certain situations are also provided to illustrate the two methods' efficiency.

This paper is organized as follows. Section 2 gives the mathematical preliminaries and states the main problem. Section 3 presents the proposed parallel subgradient method for solving the main problem and describes its convergence properties for a constant step size and for a diminishing step size and the rates of convergence under certain situations. Section 4 presents the proposed incremental subgradient method for solving the main problem and describes its convergence properties for a constant step size and for a diminishing step size and the rates of convergence under certain situations. Section 5 considers a nonsmooth convex optimization problem over the intersection of sublevel sets of convex functions and compares numerically the behaviors of the two methods with that of a previous method. Section 6 concludes the paper with a brief summary and mentions future directions for improving the proposed methods.

\section{Mathematical Preliminaries}

Let $H$ be a real Hilbert space with inner product $\langle\cdot, \cdot\rangle$ and its induced norm $\|\cdot\|$. Let $\mathbb{N}$ denote the set of all positive integers including zero. The identity mapping on $H$ is denoted by $\operatorname{Id}$; i.e., $\operatorname{Id}(x):=x(x \in H)$.

\subsection{Nonexpansivity, demiclosedness, convexity, and sub- differentiability}

The fixed point set of a mapping $Q: H \rightarrow H$ is denoted by $\operatorname{Fix}(Q):=\{x \in H: Q(x)=x\}$. $Q: H \rightarrow H$ is said to be quasi-nonexpansive [3, Definition 4.1(iii)] if $\|Q(x)-y\| \leq\|x-y\|$ for all $x \in H$ and for all $y \in \operatorname{Fix}(Q)$. When a quasi-nonexpansive mapping has one fixed point, its fixed point set is closed and convex [2, Proposition 2.6]. $Q: H \rightarrow H$ is said to be 
quasi-firmly nonexpansive [1, Section 3] if $\|Q(x)-y\|^{2}+\|(\operatorname{Id}-Q)(x)\|^{2} \leq\|x-y\|^{2}$ for all $x \in H$ and for all $y \in \operatorname{Fix}(Q)$. It is observed that any quasi-firmly nonexpansive mapping satisfies the quasi-nonexpansivity condition. It is proven from [3, Proposition 4.2] that $Q$ is quasi-firmly nonexpansive if and only if $R:=2 Q-\mathrm{Id}$ is quasi-nonexpansive. This means that $(1 / 2)(\mathrm{Id}+R)$ is quasi-firmly nonexpansive when $R$ is quasi-nonexpansive.

$Q: H \rightarrow H$ is said to be nonexpansive [3, Definition 4.1(ii)] if $\|Q(x)-Q(y)\| \leq\|x-y\|$ for all $x, y \in H$. It is obvious that any nonexpansive mapping satisfies the quasi-nonexpansivity condition. The metric projection [3, Subchapter 4.2, Chapter 28] onto a nonempty, closed convex set $C(\subset H)$, denoted by $P_{C}$, is defined for all $x \in H$ by $P_{C}(x) \in C$ and $\left\|x-P_{C}(x)\right\|=$ $\inf _{y \in C}\|x-y\| . P_{C}$ is nonexpansive with $\operatorname{Fix}\left(P_{C}\right)=C$ [3, Proposition 4.8, (4.8)].

$T: H \rightarrow H$ is referred to as a demiclosed mapping [12, p.108], [3, Theorem 4.17] if, for any $\left(x_{n}\right)_{n \in \mathbb{N}}(\subset H)$, the following implication holds: the weak convergence of $\left(x_{n}\right)_{n \in \mathbb{N}}$ to $x \in H$ and $\lim _{n \rightarrow \infty}\left\|T\left(x_{n}\right)-w\right\|=0(w \in H)$ imply $T(x)=w$. Section $[5$ will provide an example of mappings satisfying both quasi-firm nonexpansivity and demiclosedness conditions.

The following proposition indicates the properties of quasi-firmly nonexpansive mappings.

Proposition 2.1. Suppose that $Q: H \rightarrow H$ is quasi-firmly nonexpansive with $\operatorname{Fix}(Q) \neq \emptyset$ and $\alpha \in[0,1)$ and that $Q_{\alpha}:=\alpha \operatorname{Id}+(1-\alpha) Q$. Then, the following hold:

(i) $\operatorname{Fix}(Q)=\operatorname{Fix}\left(Q_{\alpha}\right)$.

(ii) $Q_{\alpha}$ is quasi-nonexpansive.

(iii) $\left\langle x-Q_{\alpha}(x), x-y\right\rangle \geq(1-\alpha)\|x-Q(x)\|^{2}(x \in H, y \in \operatorname{Fix}(Q))$ 1

Proof. Remarks 2.1(i0) and (i1) in 23 imply Proposition 2.1(i) and (ii). From $\|x-y\|^{2}=$ $\|x\|^{2}-2\langle x, y\rangle+\|y\|^{2}(x, y \in H)$, it is found that, for all $x \in H$ and for all $y \in \operatorname{Fix}(Q)$, $\langle x-Q(x), x-y\rangle=(1 / 2)\left(\|x-Q(x)\|^{2}+\|x-y\|^{2}-\|Q(x)-y\|^{2}\right)$, which, together with the quasifirm nonexpansivity of $Q$, implies that $\langle x-Q(x), x-y\rangle \geq\|x-Q(x)\|^{2}$. Hence, for all $x \in H$ and for all $y \in \operatorname{Fix}(Q),\left\langle x-Q_{\alpha}(x), x-y\right\rangle=(1-\alpha)\langle x-Q(x), x-y\rangle \geq(1-\alpha)\|x-Q(x)\|^{2}$.

A function $f: H \rightarrow \mathbb{R}$ is said to be strictly convex [3, Definition 8.6] if, for all $x, y \in H$ and for all $\alpha \in(0,1), x \neq y$ implies $f(\alpha x+(1-\alpha) y)<\alpha f(x)+(1-\alpha) f(y)$. $f$ is strongly convex with constant $\beta$ [3, Definition 10.5] if there exists $\beta>0$ such that, for all $x, y \in H$ and for all $\alpha \in(0,1), f(\alpha x+(1-\alpha) y)+(\beta / 2) \alpha(1-\alpha)\|x-y\|^{2} \leq \alpha f(x)+(1-\alpha) f(y)$.

The subdifferential [3, Definition 16.1], [32, Section 23] of $f: H \rightarrow \mathbb{R}$ is defined for all $x \in H$ by

$$
\partial f(x):=\{u \in H: f(y) \geq f(x)+\langle y-x, u\rangle \quad(y \in H)\} .
$$

We call $u(\in \partial f(x))$ the subgradient of $f$ at $x \in H$. If $f$ is strictly convex, $\partial f$ is strictly monotone; i.e., $\langle x-y, u-v\rangle>0(x, y \in H$ with $x \neq y, u \in \partial f(x), v \in \partial f(y))$ [3, Example 22.3(ii)]. If $f$ is strongly convex with constant $\beta, \partial f$ is strongly monotone; i.e., $\langle x-y, u-v\rangle \geq \beta\|x-y\|^{2}(x, y \in H, u \in \partial f(x), v \in \partial f(y))$ [3, Example 22.3(iv)].

\footnotetext{
${ }^{1}$ If $Q$ is quasi-nonexpansive, $\langle x-Q(x), x-y\rangle \geq(1 / 2)\|x-Q(x)\|^{2}(x \in H, y \in \operatorname{Fix}(Q))$. Hence, $\left\langle x-Q_{\alpha}(x), x-y\right\rangle \geq((1-\alpha) / 2)\|x-Q(x)\|^{2}(x \in H, y \in \operatorname{Fix}(Q))$. We need to use the property in Proposition 2.1(iii) to prove Lemmas 3.1 and 4.1 Accordingly, it is assumed that each user has a quasi-firmly nonexpansive mapping (see (A1)).
} 
Proposition 2.2. 3, Propositions 16.14(ii), (iii)] Let $f: H \rightarrow \mathbb{R}$ be continuous and convex with $\operatorname{dom}(f):=\{x \in H: f(x)<\infty\}=H$. Then, $\partial f(x) \neq \emptyset$ for all $x \in H$. Moreover, for all $x \in H$, there exists $\delta>0$ such that $\partial f(B(x ; \delta))$ is bounded, where $B(x ; \delta)$ stands for a closed ball with center $x$ and radius $\delta$.

The following proposition is used to prove the main results in the paper.

Proposition 2.3. 23, Lemma 2.1] Let $\left(\Gamma_{n}\right)_{n \in \mathbb{N}} \subset \mathbb{R}$ and suppose that $\left(\Gamma_{n_{j}}\right)_{j \in \mathbb{N}}\left(\subset\left(\Gamma_{n}\right)_{n \in \mathbb{N}}\right)$ exists such that $\Gamma_{n_{j}}<\Gamma_{n_{j}+1}$ for all $j \in \mathbb{N}$. Define $(\tau(n))_{n \geq n_{0}} \subset \mathbb{N}$ by $\tau(n):=\max \{k \leq$ $\left.n: \Gamma_{k}<\Gamma_{k+1}\right\}$ for some $n_{0} \in \mathbb{N}$. Then, $(\tau(n))_{n \geq n_{0}}$ is increasing and $\lim _{n \rightarrow \infty} \tau(n)=\infty$. Moreover, $\Gamma_{\tau(n)} \leq \Gamma_{\tau(n)+1}$ and $\Gamma_{n} \leq \Gamma_{\tau(n)+1}$ for all $n \geq n_{0}$.

\subsection{Main problem}

The focus here is a networked system with $I$ users. Let

$$
\mathcal{I}:=\{1,2, \ldots, I\}
$$

Suppose that user $i(i \in \mathcal{I})$ has its own private objective function, denoted by $f^{(i)}: H \rightarrow \mathbb{R}$, and its own mapping, denoted by $Q^{(i)}: H \rightarrow H$. The following notation is used.

$$
\begin{aligned}
& Q_{\alpha}^{(i)}:=\alpha^{(i)} \operatorname{Id}+\left(1-\alpha^{(i)}\right) Q^{(i)}\left(\alpha^{(i)} \in(0,1)\right), X:=\bigcap_{i \in \mathcal{I}} \operatorname{Fix}\left(Q^{(i)}\right), \\
& f:=\sum_{i \in \mathcal{I}} f^{(i)}, X^{\star}:=\left\{x \in X: f(x)=f^{\star}:=\inf _{y \in X} f(y)\right\} .
\end{aligned}
$$

The following problem is discussed in this paper.

Problem 2.1. Suppose that the following (A1)-(A4) hold.

(A1) $Q^{(i)}: H \rightarrow H(i \in \mathcal{I})$ is quasi-firmly nonexpansive.

(A2) $f^{(i)}: H \rightarrow \mathbb{R}(i \in \mathcal{I})$ is continuous and convex with $\operatorname{dom}\left(f^{(i)}\right)=H 2$

(A3) User $i(i \in \mathcal{I})$ can use its own private $Q^{(i)}$ and $\partial f^{(i)}$.

(A4) $X^{\star} \neq \emptyset$.

Then, find $x^{\star} \in X^{\star}$.

\section{Parallel Subgradient Method}

The section presents a method for solving Problem 2.1 under the assumption that

(A5) each user can communicate with other users.

\footnotetext{
${ }^{2}$ When $H=\mathbb{R}^{N}$, a convex function $f^{(i)}$ satisfies the continuity condition 3. Corollary 8.31]. Therefore, (A2) can be replaced by the convexity condition of $f^{(i)}$ with $\operatorname{dom}\left(f^{(i)}\right)=$ $\mathbb{R}^{N}$.
} 


\section{Algorithm 3.1.}

Step 0. User $i(i \in \mathcal{I})$ sets $\alpha^{(i)},\left(\lambda_{n}\right)_{n \in \mathbb{N}} \subset(0, \infty)$, and $x_{0} \in H$.

Step 1. User $i(i \in \mathcal{I})$ computes $x_{n}^{(i)} \in H$ using

$$
x_{n}^{(i)}:=Q_{\alpha}^{(i)}\left(x_{n}\right)-\lambda_{n} g_{n}^{(i)} \text {, where } g_{n}^{(i)} \in \partial f^{(i)}\left(Q_{\alpha}^{(i)}\left(x_{n}\right)\right) .
$$

User $i(i \in \mathcal{I})$ transmits $x_{n}^{(i)}$ to all users.

Step 2. User $i(i \in \mathcal{I})$ computes $x_{n+1} \in H$ as

$$
x_{n+1}:=\frac{1}{I} \sum_{i \in \mathcal{I}} x_{n}^{(i)} .
$$

The algorithm sets $n:=n+1$ and returns to Step 1 .

Algorithm 3.1 requires that all users set the same step-size sequence $\left(\lambda_{n}\right)_{n \in \mathbb{N}}$ before algorithm execution and that they synchronize at each iteration. See [31, Section 6] for the asynchronous distributed proximal algorithm that was used for solving nonsmooth convex optimization. Assumption (A5) ensures that each user has access to all $x_{n}^{(i)}$ and can compute $x_{n+1}=(1 / I) \sum_{i \in \mathcal{I}} x_{n}^{(i)}$. This means that a common variable $x_{n}(n \in \mathbb{N})$ is shared by all users. To illustrate this situation, let us assume that there exists an operator who manages the system. Even in a situation where (A5) is not satisfied, the operator can still communicate with all users. Accordingly, if the operator sets an initial point $x_{0}$ and transmits $x_{n}$ to all users at each iteration $n$, user $i$ can compute $x_{n}^{(i)}$ by using its own private information. Since the operator has access to all $x_{n}^{(i)}$, the operator can compute $x_{n+1}$ and transmit it to all users. Therefore, assuming the existence of an operator guarantees that all users can share $x_{n}(n \in \mathbb{N})$ in Algorithm 3.1.

The convergence of Algorithm 3.1 depends on two assumptions.

Assumption 3.1. For all $i \in \mathcal{I}$, there exist $M_{1}^{(i)}, M_{2}^{(i)} \in \mathbb{R}$ such that

$$
\begin{aligned}
& \sup \left\{\|g\|: g \in \partial f^{(i)}\left(Q_{\alpha}^{(i)}\left(x_{n}\right)\right), n \in \mathbb{N}\right\} \leq M_{1}^{(i)}, \\
& \sup \left\{\|g\|: g \in \partial f^{(i)}\left(x_{n}\right), n \in \mathbb{N}\right\} \leq M_{2}^{(i)} .
\end{aligned}
$$

Assumption 3.2. The sequence $\left(x_{n}^{(i)}\right)_{n \in \mathbb{N}}(i \in \mathcal{I})$ is bounded.

Assumption 3.2 implies Assumption 3.1. Indeed, the definition of $x_{n}(n \in \mathbb{N})$ and the boundedness of $\left(x_{n}^{(i)}\right)_{n \in \mathbb{N}}(i \in \mathcal{I})$ ensure that $\left(x_{n}\right)_{n \in \mathbb{N}}$ is bounded. From the quasinonexpansivity of $Q_{\alpha}^{(i)}(i \in \mathcal{I})$, we have $\left\|Q_{\alpha}^{(i)}\left(x_{n}\right)-x\right\| \leq\left\|x_{n}-x\right\|(x \in X)$, which, together with the boundedness of $\left(x_{n}\right)_{n \in \mathbb{N}}$, means that $\left(Q_{\alpha}^{(i)}\left(x_{n}\right)\right)_{n \in \mathbb{N}}(i \in \mathcal{I})$ is bounded. Hence, Proposition 2.2 implies that Assumption 3.1 holds.

A convergence analysis of Algorithm 3.1 with a constant step size when Assumption 3.1 holds is given in Subsection 3.1. The discussion in Subsection 3.2 needs to satisfy Assumption 3.2. which is stronger than Assumption 3.1, to enable the convergence property of Algorithm 3.1 with a diminishing step-size sequence to be studied. This is because, in the case where Assumption 3.2 does not hold and $\left(\left\|x_{n}-x\right\|\right)_{n \in \mathbb{N}}(x \in X)$ is not monotone decreasing (see Case 2 in the proof of Theorem 3.21), a weak convergent subsequence of $\left(x_{n}\right)_{n \in \mathbb{N}}$ does not exist; i.e., we cannot discuss weak convergence of Algorithm 3.1 to a point in $X^{\star}$. 
Here we provide an example satisfying Assumption 3.2 and (A4). Let us assume that user $i(i \in \mathcal{I})$ can choose in advance a simple, bounded, closed convex set $X^{(i)}$ (e.g., $X^{(i)}$ is a closed ball with a large enough radius) satisfying $X^{(i)} \supset \operatorname{Fix}\left(Q^{(i)}\right)$. Then, user $i$ can compute $P^{(i)}:=P_{X^{(i)}}$ and

$$
x_{n}^{(i)}:=P^{(i)}\left(Q_{\alpha}^{(i)}\left(x_{n}\right)-\lambda_{n} g_{n}^{(i)}\right)
$$

instead of $x_{n}^{(i)}$ in Algorithm 3.1. Since $\left(x_{n}^{(i)}\right)_{n \in \mathbb{N}} \subset X^{(i)}$ and $X^{(i)}$ is bounded, $\left(x_{n}^{(i)}\right)_{n \in \mathbb{N}}$ is bounded. Since $X^{(i)}$ is bounded and $X \subset X^{(i)}(i \in \mathcal{I}), X$ is also bounded. Hence, the continuity and convexity of $f$ ensure that $X^{\star} \neq \emptyset$; i.e., (A4) holds [3, Proposition 11.14]. We can show that Algorithm 3.1 with (3.1) satisfies the convergence properties in the main theorems in this paper by referring to the proofs of the theorems.

The following is an important lemma that will be used to prove the main theorems.

Lemma 3.1. Suppose that $\left(x_{n}\right)_{n \in \mathbb{N}}$ is the sequence generated by Algorithm [3.1 and that Assumptions (A1)-(A5) and 3.1 hold. The following properties then hold:

(i) For all $n \in \mathbb{N}$ and for all $x \in X$,

$$
\begin{aligned}
\left\|x_{n+1}-x\right\|^{2} \leq & \left\|x_{n}-x\right\|^{2}-\frac{2}{I} \sum_{i \in \mathcal{I}} \alpha^{(i)}\left(1-\alpha^{(i)}\right)\left\|x_{n}-Q^{(i)}\left(x_{n}\right)\right\|^{2} \\
& +2 M_{1} \lambda_{n}^{2}-\frac{2 \lambda_{n}}{I} \sum_{i \in \mathcal{I}}\left\langle x_{n}-x, g_{n}^{(i)}\right\rangle
\end{aligned}
$$

where $M_{1}:=\max _{i \in \mathcal{I}} M_{1}^{(i)^{2}}<\infty$.

(ii) For all $n \in \mathbb{N}$ and for all $x \in X$,

$$
\begin{aligned}
\left\|x_{n+1}-x\right\|^{2} \leq & \left\|x_{n}-x\right\|^{2}+2 M_{1} \lambda_{n}^{2}+\frac{2 \lambda_{n}}{I}\left(f(x)-f\left(x_{n}\right)\right) \\
& +\frac{2 \lambda_{n}}{I}\left(\sqrt{M_{1}}+M_{2}\right) \sum_{i \in \mathcal{I}}\left\|x_{n}-Q_{\alpha}^{(i)}\left(x_{n}\right)\right\|,
\end{aligned}
$$

where $M_{2}:=\max _{i \in \mathcal{I}} M_{2}^{(i)}<\infty$.

Proof. (i) Choose $x \in X \subset \operatorname{Fix}\left(Q^{(i)}\right)(i \in \mathcal{I})$ and $n \in \mathbb{N}$ arbitrarily. From $-2\langle x, y\rangle=$ $\|x-y\|^{2}-\|x\|^{2}-\|y\|^{2}(x, y \in H)$, we find that, for all $i \in \mathcal{I}$,

$$
\begin{aligned}
& 2\left\langle x_{n}^{(i)}-x_{n}+\lambda_{n} g_{n}^{(i)}, x_{n}-x\right\rangle \\
= & -2\left\langle x_{n}-x_{n}^{(i)}, x_{n}-x\right\rangle+2 \lambda_{n}\left\langle x_{n}-x, g_{n}^{(i)}\right\rangle \\
= & \left\|x_{n}^{(i)}-x\right\|^{2}-\left\|x_{n}-x_{n}^{(i)}\right\|^{2}-\left\|x_{n}-x\right\|^{2}+2 \lambda_{n}\left\langle x_{n}-x, g_{n}^{(i)}\right\rangle .
\end{aligned}
$$

Moreover, Proposition 2.1(iii) ensures that, for all $i \in \mathcal{I}$,

$$
2\left\langle Q_{\alpha}^{(i)}\left(x_{n}\right)-x_{n}, x_{n}-x\right\rangle \leq-2\left(1-\alpha^{(i)}\right)\left\|x_{n}-Q^{(i)}\left(x_{n}\right)\right\|^{2} .
$$


Accordingly, from $x_{n}^{(i)}:=Q_{\alpha}^{(i)}\left(x_{n}\right)-\lambda_{n} g_{n}^{(i)}(i \in \mathcal{I})$,

$$
\begin{aligned}
2\left\langle x_{n}^{(i)}-x_{n}+\lambda_{n} g_{n}^{(i)}, x_{n}-x\right\rangle & =2\left\langle Q_{\alpha}^{(i)}\left(x_{n}\right)-x_{n}, x_{n}-x\right\rangle \\
& \leq-2\left(1-\alpha^{(i)}\right)\left\|x_{n}-Q^{(i)}\left(x_{n}\right)\right\|^{2} .
\end{aligned}
$$

Therefore, for all $i \in \mathcal{I}$,

$$
\begin{aligned}
\left\|x_{n}^{(i)}-x\right\|^{2} \leq & \left\|x_{n}-x\right\|^{2}+\left\|x_{n}-x_{n}^{(i)}\right\|^{2}-2 \lambda_{n}\left\langle x_{n}-x, g_{n}^{(i)}\right\rangle \\
& -2\left(1-\alpha^{(i)}\right)\left\|x_{n}-Q^{(i)}\left(x_{n}\right)\right\|^{2}
\end{aligned}
$$

Moreover, from $\|x-y\|^{2} \leq 2\|x\|^{2}+2\|y\|^{2}(x, y \in H)$,

$$
\begin{aligned}
\left\|x_{n}-x_{n}^{(i)}\right\|^{2} & =\left\|\left(x_{n}-Q_{\alpha}^{(i)}\left(x_{n}\right)\right)+\lambda_{n} g_{n}^{(i)}\right\|^{2} \\
& \leq 2\left\|x_{n}-Q_{\alpha}^{(i)}\left(x_{n}\right)\right\|^{2}+2 \lambda_{n}^{2}\left\|g_{n}^{(i)}\right\|^{2} \\
& \leq 2\left(1-\alpha^{(i)}\right)^{2}\left\|x_{n}-Q^{(i)}\left(x_{n}\right)\right\|^{2}+2 M_{1} \lambda_{n}^{2}
\end{aligned}
$$

where $M_{1}:=\max _{i \in \mathcal{I}} M_{1}^{(i)^{2}}<\infty$ holds from Assumption 3.1. Hence, for all $i \in \mathcal{I}$,

$$
\begin{aligned}
\left\|x_{n}^{(i)}-x\right\|^{2} \leq & \left\|x_{n}-x\right\|^{2}-2 \alpha^{(i)}\left(1-\alpha^{(i)}\right)\left\|x_{n}-Q^{(i)}\left(x_{n}\right)\right\|^{2}+2 M_{1} \lambda_{n}^{2} \\
& -2 \lambda_{n}\left\langle x_{n}-x, g_{n}^{(i)}\right\rangle
\end{aligned}
$$

which, together with the convexity of $\|\cdot\|^{2}$, implies that

$$
\begin{aligned}
\left\|x_{n+1}-x\right\|^{2} \leq & \frac{1}{I} \sum_{i \in \mathcal{I}}\left\|x_{n}^{(i)}-x\right\|^{2} \\
\leq & \left\|x_{n}-x\right\|^{2}-\frac{2}{I} \sum_{i \in \mathcal{I}} \alpha^{(i)}\left(1-\alpha^{(i)}\right)\left\|x_{n}-Q^{(i)}\left(x_{n}\right)\right\|^{2}+2 M_{1} \lambda_{n}^{2} \\
& -\frac{2 \lambda_{n}}{I} \sum_{i \in \mathcal{I}}\left\langle x_{n}-x, g_{n}^{(i)}\right\rangle .
\end{aligned}
$$

(ii) Choose $x \in X \subset \operatorname{Fix}\left(Q^{(i)}\right)(i \in \mathcal{I})$ and $n \in \mathbb{N}$ arbitrarily. From $g_{n}^{(i)} \in \partial f^{(i)}\left(Q_{\alpha}^{(i)}\left(x_{n}\right)\right)$ $(i \in \mathcal{I}),\left\langle x-Q_{\alpha}^{(i)}\left(x_{n}\right), g_{n}^{(i)}\right\rangle \leq f^{(i)}(x)-f^{(i)}\left(Q_{\alpha}^{(i)}\left(x_{n}\right)\right)$. Hence, the Cauchy-Schwarz inequality ensures that, for all $i \in \mathcal{I},\left\langle x-x_{n}, g_{n}^{(i)}\right\rangle=\left\langle x-Q_{\alpha}^{(i)}\left(x_{n}\right), g_{n}^{(i)}\right\rangle+\left\langle Q_{\alpha}^{(i)}\left(x_{n}\right)-x_{n}, g_{n}^{(i)}\right\rangle \leq$ $f^{(i)}(x)-f^{(i)}\left(Q_{\alpha}^{(i)}\left(x_{n}\right)\right)+\sqrt{M_{1}}\left\|Q_{\alpha}^{(i)}\left(x_{n}\right)-x_{n}\right\|$, which, together with $f:=\sum_{i \in \mathcal{I}} f^{(i)}$, implies that

$$
\begin{aligned}
\sum_{i \in \mathcal{I}}\left\langle x-x_{n}, g_{n}^{(i)}\right\rangle \leq & f(x)-f\left(x_{n}\right)+\sum_{i \in \mathcal{I}}\left(f^{(i)}\left(x_{n}\right)-f^{(i)}\left(Q_{\alpha}^{(i)}\left(x_{n}\right)\right)\right) \\
& +\sqrt{M_{1}} \sum_{i \in \mathcal{I}}\left\|Q_{\alpha}^{(i)}\left(x_{n}\right)-x_{n}\right\| .
\end{aligned}
$$


Set $M_{2}:=\max _{i \in \mathcal{I}} M_{2}^{(i)}$. Then, Assumption 3.1 ensures that $M_{2}<\infty$. Since $g \in \partial f^{(i)}\left(x_{n}\right)$ implies that, for all $i \in \mathcal{I}, f^{(i)}\left(x_{n}\right)-f^{(i)}\left(Q_{\alpha}^{(i)}\left(x_{n}\right)\right) \leq\left\langle x_{n}-Q_{\alpha}^{(i)}\left(x_{n}\right), g\right\rangle \leq M_{2}\left\|x_{n}-Q_{\alpha}^{(i)}\left(x_{n}\right)\right\|$, it is found that

$$
\sum_{i \in \mathcal{I}}\left\langle x-x_{n}, g_{n}^{(i)}\right\rangle \leq f(x)-f\left(x_{n}\right)+\left(\sqrt{M_{1}}+M_{2}\right) \sum_{i \in \mathcal{I}}\left\|Q_{\alpha}^{(i)}\left(x_{n}\right)-x_{n}\right\| .
$$

Accordingly, Lemma 3.1(i) leads to Lemma 3.1(ii). This completes the proof.

\subsection{Constant step-size rule}

The discussion in this subsection is based on the following assumption.

Assumption 3.3. User $i(i \in \mathcal{I})$ has $\left(\lambda_{n}\right)_{n \in \mathbb{N}}$ satisfying

$$
\text { (C1) } \lambda_{n}:=\lambda \in(0, \infty)(n \in \mathbb{N}) \text {. }
$$

Let us perform a convergence analysis of Algorithm 3.1 under Assumption 3.3 .

Theorem 3.1. Suppose that Assumptions (A1)-(A5), 3.1, and 3.3 hold. Then, $\left(x_{n}\right)_{n \in \mathbb{N}}$ in Algorithm 3.1] satisfies the relations

$$
\begin{aligned}
& \liminf _{n \rightarrow \infty}\left\|x_{n}-Q^{(i)}\left(x_{n}\right)\right\|^{2} \leq \frac{I M_{\lambda} \lambda}{\alpha^{(i)}\left(1-\alpha^{(i)}\right)}(i \in \mathcal{I}), \\
& \liminf _{n \rightarrow \infty} f\left(x_{n}\right) \leq f^{\star}+I M_{1} \lambda+\left(\sqrt{M_{1}}+M_{2}\right) \sum_{i \in \mathcal{I}} \sqrt{\frac{\left(1-\alpha^{(i)}\right) I M_{\lambda} \lambda}{\alpha^{(i)}}},
\end{aligned}
$$

where $M_{1}$ and $M_{2}$ are as in Lemma 3.1 and, for some $x \in X, M_{\lambda}:=\sup _{n \in \mathbb{N}}\left(M_{1} \lambda+\right.$ $\left.(1 / I)\left|\sum_{i \in \mathcal{I}}\left\langle x-x_{n}, g_{n}^{(i)}\right\rangle\right|\right)$.

Theorem 3.1 implies that Algorithm 3.1 with a small enough $\lambda$ may approximate a solution to Problem 2.1.

Proof. Choose $x \in X$ arbitrarily and set $M_{\lambda}:=\sup _{n \in \mathbb{N}}\left(M_{1} \lambda+(1 / I)\left|\sum_{i \in \mathcal{I}}\left\langle x-x_{n}, g_{n}^{(i)}\right\rangle\right|\right)$. It is obvious that Theorem 3.1 holds when $M_{\lambda}=\infty$. Consider the case where $M_{\lambda}<\infty$. First, let us show that

$$
\liminf _{n \rightarrow \infty} \sum_{i \in \mathcal{I}} \alpha^{(i)}\left(1-\alpha^{(i)}\right)\left\|x_{n}-Q^{(i)}\left(x_{n}\right)\right\|^{2} \leq I M_{\lambda} \lambda .
$$

Here we assume that (3.2) does not hold. Accordingly, $\delta(>0)$ can be chosen such that

$$
\liminf _{n \rightarrow \infty} \sum_{i \in \mathcal{I}} \alpha^{(i)}\left(1-\alpha^{(i)}\right)\left\|x_{n}-Q^{(i)}\left(x_{n}\right)\right\|^{2}>I M_{\lambda} \lambda+2 \delta .
$$

The property of the limit inferior of $\left(\sum_{i \in \mathcal{I}} \alpha^{(i)}\left(1-\alpha^{(i)}\right)\left\|x_{n}-Q^{(i)}\left(x_{n}\right)\right\|^{2}\right)_{n \in \mathbb{N}}$ guarantees that there exists $n_{0} \in \mathbb{N}$ such that $\liminf _{n \rightarrow \infty} \sum_{i \in \mathcal{I}} \alpha^{(i)}\left(1-\alpha^{(i)}\right)\left\|x_{n}-Q^{(i)}\left(x_{n}\right)\right\|^{2}-\delta \leq$ $\sum_{i \in \mathcal{I}} \alpha^{(i)}\left(1-\alpha^{(i)}\right)\left\|x_{n}-Q^{(i)}\left(x_{n}\right)\right\|^{2}$ for all $n \geq n_{0}$. Accordingly, for all $n \geq n_{0}$,

$$
\sum_{i \in \mathcal{I}} \alpha^{(i)}\left(1-\alpha^{(i)}\right)\left\|x_{n}-Q^{(i)}\left(x_{n}\right)\right\|^{2}>I M_{\lambda} \lambda+\delta .
$$


Hence, Lemma 3.1(i) leads to the finding that, for all $n \geq n_{0}$,

$$
\begin{aligned}
\left\|x_{n+1}-x\right\|^{2} & \leq\left\|x_{n}-x\right\|^{2}-\sum_{i \in \mathcal{I}} \frac{2 \alpha^{(i)}\left(1-\alpha^{(i)}\right)}{I}\left\|x_{n}-Q^{(i)}\left(x_{n}\right)\right\|^{2}+2 M_{\lambda} \lambda \\
& <\left\|x_{n}-x\right\|^{2}-\frac{2}{I}\left\{I M_{\lambda} \lambda+\delta\right\}+2 M_{\lambda} \lambda \\
& =\left\|x_{n}-x\right\|^{2}-\frac{2}{I} \delta
\end{aligned}
$$

Therefore, induction ensures that, for all $n \geq n_{0}$,

$$
0 \leq\left\|x_{n+1}-x\right\|^{2}<\left\|x_{n_{0}}-x\right\|^{2}-\frac{2}{I} \delta\left(n+1-n_{0}\right) .
$$

Since the right side of this inequality approaches minus infinity as $n$ diverges, there is a contradiction. Therefore, (3.2) holds. Since $\liminf _{n \rightarrow \infty} \alpha^{(i)}\left(1-\alpha^{(i)}\right)\left\|x_{n}-Q^{(i)}\left(x_{n}\right)\right\|^{2} \leq$ $\liminf _{n \rightarrow \infty} \sum_{i \in \mathcal{I}} \alpha^{(i)}\left(1-\alpha^{(i)}\right)\left\|x_{n}-Q^{(i)}\left(x_{n}\right)\right\|^{2}(i \in \mathcal{I})$, there is also another finding:

$$
\liminf _{n \rightarrow \infty}\left\|x_{n}-Q^{(i)}\left(x_{n}\right)\right\|^{2} \leq \frac{I M_{\lambda} \lambda}{\alpha^{(i)}\left(1-\alpha^{(i)}\right)}(i \in \mathcal{I}) .
$$

Let $i \in \mathcal{I}$ be fixed arbitrarily. Inequality (3.3) and the property of the limit inferior of $\left(\left\|x_{n}-Q^{(i)}\left(x_{n}\right)\right\|^{2}\right)_{n \in \mathbb{N}}$ guarantee the existence of a subsequence $\left(x_{n_{k}}\right)_{k \in \mathbb{N}}$ of $\left(x_{n}\right)_{n \in \mathbb{N}}$ such that

$$
\lim _{k \rightarrow \infty}\left\|x_{n_{k}}-Q^{(i)}\left(x_{n_{k}}\right)\right\|^{2}=\liminf _{n \rightarrow \infty}\left\|x_{n}-Q^{(i)}\left(x_{n}\right)\right\|^{2} \leq \frac{I M_{\lambda} \lambda}{\alpha^{(i)}\left(1-\alpha^{(i)}\right)} .
$$

Therefore, for all $\epsilon>0$, there exists $k_{0} \in \mathbb{N}$ such that, for all $k \geq k_{0}$,

$$
\left\|x_{n_{k}}-Q^{(i)}\left(x_{n_{k}}\right)\right\| \leq \sqrt{\frac{I M_{\lambda} \lambda}{\alpha^{(i)}\left(1-\alpha^{(i)}\right)}+\epsilon} .
$$

Here, it is proven that, for all $k \geq k_{0}$,

$$
\liminf _{n \rightarrow \infty} f\left(x_{n}\right) \leq f^{\star}+I M_{1} \lambda+\left(\sqrt{M_{1}}+M_{2}\right) \sum_{i \in \mathcal{I}}\left\|x_{n_{k}}-Q_{\alpha}^{(i)}\left(x_{n_{k}}\right)\right\|+2 \epsilon .
$$

Now, let us assume that (3.5) does not hold for all $k \geq k_{0}$, i.e., there exists $n_{1} \in \mathbb{N}$ such that, for all $n \geq n_{1}$,

$$
\liminf _{n \rightarrow \infty} f\left(x_{n}\right)>f^{\star}+I M_{1} \lambda+\left(\sqrt{M_{1}}+M_{2}\right) \sum_{i \in \mathcal{I}}\left\|x_{n}-Q_{\alpha}^{(i)}\left(x_{n}\right)\right\|+2 \epsilon .
$$

Assumption (A4) means the existence of $x^{\star} \in X$ such that $f\left(x^{\star}\right)=f^{\star}$. Since the property of the limit inferior of $\left(f\left(x_{n}\right)\right)_{n \in \mathbb{N}}$ implies the existence of $n_{2} \in \mathbb{N}$ such that $\lim _{\inf } \operatorname{si\infty }_{n \rightarrow \infty} f\left(x_{n}\right)$ $\epsilon \leq f\left(x_{n}\right)$ for all $n \geq n_{2}$, it is found that, for all $n \geq n_{3}:=\max \left\{n_{1}, n_{2}\right\}$,

$$
f\left(x_{n}\right)-f\left(x^{\star}\right)>I M_{1} \lambda+\left(\sqrt{M_{1}}+M_{2}\right) \sum_{i \in \mathcal{I}}\left\|x_{n}-Q_{\alpha}^{(i)}\left(x_{n}\right)\right\|+\epsilon .
$$


Therefore, Lemma 3.1(ii) guarantees that, for all $n \geq n_{3}$,

$$
\begin{aligned}
\left\|x_{n+1}-x^{\star}\right\|^{2} \leq & \left\|x_{n}-x^{\star}\right\|^{2}+2 M_{1} \lambda^{2}+\frac{2 \lambda}{I}\left(f\left(x^{\star}\right)-f\left(x_{n}\right)\right) \\
& +\frac{2 \lambda}{I}\left(\sqrt{M_{1}}+M_{2}\right) \sum_{i \in \mathcal{I}}\left\|x_{n}-Q_{\alpha}^{(i)}\left(x_{n}\right)\right\| \\
< & \left\|x_{n}-x^{\star}\right\|^{2}+2 M_{1} \lambda^{2} \\
& -\frac{2 \lambda}{I}\left\{I M_{1} \lambda+\left(\sqrt{M_{1}}+M_{2}\right) \sum_{i \in \mathcal{I}}\left\|x_{n}-Q_{\alpha}^{(i)}\left(x_{n}\right)\right\|+\epsilon\right\} \\
& +\frac{2 \lambda}{I}\left(\sqrt{M_{1}}+M_{2}\right) \sum_{i \in \mathcal{I}}\left\|x_{n}-Q_{\alpha}^{(i)}\left(x_{n}\right)\right\| \\
= & \left\|x_{n}-x^{\star}\right\|^{2}-\frac{2 \lambda}{I} \epsilon \\
< & \left\|x_{n_{3}}-x^{\star}\right\|^{2}-\frac{2 \lambda}{I} \epsilon\left(n+1-n_{3}\right) .
\end{aligned}
$$

Since the right side of the above inequality approaches minus infinity as $n$ diverges, there is a contradiction. Thus, (3.5) holds for all $k \geq k_{0}$. Therefore, (3.4) and (3.5) lead to the deduction that, for all $\epsilon>0$,

$$
\begin{aligned}
\liminf _{n \rightarrow \infty} f\left(x_{n}\right) \leq & f^{\star}+I M_{1} \lambda \\
& +\left(\sqrt{M_{1}}+M_{2}\right) \sum_{i \in \mathcal{I}}\left(1-\alpha^{(i)}\right) \sqrt{\frac{I M_{\lambda} \lambda}{\alpha^{(i)}\left(1-\alpha^{(i)}\right)}+\epsilon}+2 \epsilon .
\end{aligned}
$$

Since $\epsilon(>0)$ is arbitrary,

$$
\liminf _{n \rightarrow \infty} f\left(x_{n}\right) \leq f^{\star}+I M_{1} \lambda+\left(\sqrt{M_{1}}+M_{2}\right) \sum_{i \in \mathcal{I}} \sqrt{\frac{\left(1-\alpha^{(i)}\right) I M_{\lambda} \lambda}{\alpha^{(i)}}} .
$$

This completes the proof.

\subsection{Diminishing step-size rule}

The discussion in this subsection is based on the following assumption.

Assumption 3.4. User $i(i \in \mathcal{I})$ has $\left(\lambda_{n}\right)_{n \in \mathbb{N}}$ satisfying

$$
\text { (C2) } \lim _{n \rightarrow \infty} \lambda_{n}=0 \text { and (C3) } \sum_{n=0}^{\infty} \lambda_{n}=\infty \text {. }
$$

Moreover,

(A6) Id $-Q^{(i)}(i \in \mathcal{I})$ is demiclosed.

An example of $\left(\lambda_{n}\right)_{n \in \mathbb{N}}$ satisfying $(\mathrm{C} 2)$ and $(\mathrm{C} 3)$ is $\lambda_{n}:=1 /(n+1)^{a}(n \in \mathbb{N})$, where $a \in(0,1]$. Section 5 will provide an example of $Q^{(i)}(i \in \mathcal{I})$ satisfying (A1) and (A6).

Let us perform a convergence analysis of Algorithm 3.1 under Assumption 3.4 . 
Theorem 3.2. Suppose that Assumptions (A1)-(A5), 3.2, and 3.4 hold. Then there exists a subsequence of $\left(x_{n}\right)_{n \in \mathbb{N}}$ generated by Algorithm 3.1 that weakly converges to a point in $X^{\star}$. Moreover, the whole sequence $\left(x_{n}\right)_{n \in \mathbb{N}}$ strongly converges to a unique point in $X^{\star}$ if one of the following holds 3

(i) One $f^{(i)}(i \in \mathcal{I})$ is strongly convex.

(ii) $H$ is finite-dimensional, and one $f^{(i)}(i \in \mathcal{I})$ is strictly convex.

Proof. We consider two cases.

Case 1: Suppose that there exists $m_{0} \in \mathbb{N}$ such that $\left\|x_{n+1}-x^{\star}\right\| \leq\left\|x_{n}-x^{\star}\right\|$ for all $n \geq m_{0}$ and for all $x^{\star} \in X^{\star}$. The existence of $\lim _{n \rightarrow \infty}\left\|x_{n}-x^{\star}\right\|$ is thus guaranteed for all $x^{\star} \in X^{\star}$. Hence, $\left(x_{n}\right)_{n \in \mathbb{N}}$ is bounded. The quasi-nonexpansivity of $Q_{\alpha}^{(i)}(i \in \mathcal{I})$ thus ensures that $\left(Q_{\alpha}^{(i)}\left(x_{n}\right)\right)_{n \in \mathbb{N}}(i \in \mathcal{I})$ is bounded. Accordingly, Proposition 2.2 guarantees that $M_{1}$ and $M_{2}$ defined as in Lemma 3.1 are finite. From Lemma 3.1(i), for all $n \geq m_{0}$ and for all $x^{\star} \in X^{\star}$,

$$
\begin{aligned}
& \sum_{i \in \mathcal{I}} \frac{2 \alpha^{(i)}\left(1-\alpha^{(i)}\right)}{I}\left\|x_{n}-Q^{(i)}\left(x_{n}\right)\right\|^{2} \\
\leq & \left\|x_{n}-x^{\star}\right\|^{2}-\left\|x_{n+1}-x^{\star}\right\|^{2}+2 M_{1} \lambda_{n}^{2}-\frac{2 \lambda_{n}}{I} \sum_{i \in \mathcal{I}}\left\langle x_{n}-x^{\star}, g_{n}^{(i)}\right\rangle,
\end{aligned}
$$

which, together with $(\mathrm{C} 2)$ and the boundedness of $\left(g_{n}^{(i)}\right)_{n \in \mathbb{N}}(i \in \mathcal{I})$, implies that $\lim _{n \rightarrow \infty}(1 / I) \sum_{i \in \mathcal{I}} 2 \alpha^{(i)}(1-$ $\left.\alpha^{(i)}\right)\left\|x_{n}-Q^{(i)}\left(x_{n}\right)\right\|^{2}=0$; i.e.,

$$
\lim _{n \rightarrow \infty}\left\|x_{n}-Q_{\alpha}^{(i)}\left(x_{n}\right)\right\|=\lim _{n \rightarrow \infty}\left\|x_{n}-Q^{(i)}\left(x_{n}\right)\right\|=0 \quad(i \in \mathcal{I}) .
$$

Let us define, for all $n \in \mathbb{N}$ and for all $x \in X$,

$$
M_{n}(x):=f\left(x_{n}\right)-f(x)-\left(\sqrt{M_{1}}+M_{2}\right) \sum_{i \in \mathcal{I}}\left\|x_{n}-Q_{\alpha}^{(i)}\left(x_{n}\right)\right\|-I M_{1} \lambda_{n} .
$$

Then, Lemma 3.1(ii) leads to the finding that, for all $n \in \mathbb{N}$ and for all $x \in X$,

$$
\frac{2 \lambda_{n}}{I} M_{n}(x) \leq\left\|x_{n}-x\right\|^{2}-\left\|x_{n+1}-x\right\|^{2} .
$$

Summing up this inequality from $n=0$ to $n=m(m \in \mathbb{N})$ implies that $(2 / I) \sum_{n=0}^{m} \lambda_{n} M_{n}(x) \leq$ $\left\|x_{0}-x\right\|^{2}-\left\|x_{m+1}-x\right\|^{2} \leq\left\|x_{0}-x\right\|^{2}<\infty$, so

$$
\sum_{n=0}^{\infty} \lambda_{n} M_{n}(x)<\infty(x \in X) .
$$

\footnotetext{
${ }^{3}$ Under (A4), the strict convexity of $f$ guarantees the uniqueness of the solution to Problem 2.1 38, Corollary 25.15]. If there exists an operator who manages the system, it is reasonable to assume that the operator has a strongly convex objective function so as to guarantee the convergence of $\left(x_{n}\right)_{n \in \mathbb{N}}$ in Algorithm 3.1 to the desired solution, i.e., one that makes the system stable and reliable.
} 
Let us fix $x \in X$ arbitrarily. Now, under the assumption that $\liminf _{n \rightarrow \infty} M_{n}(x)>0$, $m_{1} \in \mathbb{N}$ and $\gamma>0$ can be chosen such that $M_{n}(x) \geq \gamma$ for all $n \geq m_{1}$. Accordingly, (C3) means that

$$
\infty=\gamma \sum_{n=m_{1}}^{\infty} \lambda_{n} \leq \sum_{n=m_{1}}^{\infty} \lambda_{n} M_{n}(x)<\infty
$$

which is a contradiction. Therefore, for all $x \in X, \liminf _{n \rightarrow \infty} M_{n}(x) \leq 0$, i.e.,

$$
\liminf _{n \rightarrow \infty}\left\{f\left(x_{n}\right)-f(x)-\left(\sqrt{M_{1}}+M_{2}\right) \sum_{i \in \mathcal{I}}\left\|x_{n}-Q_{\alpha}^{(i)}\left(x_{n}\right)\right\|-I M_{1} \lambda_{n}\right\} \leq 0,
$$

which, together with $(\mathrm{C} 2)$ and (3.6), implies that

$$
\liminf _{n \rightarrow \infty} f\left(x_{n}\right) \leq f(x) \quad(x \in X) .
$$

Accordingly, there exists a subsequence $\left(x_{n_{l}}\right)_{l \in \mathbb{N}}$ of $\left(x_{n}\right)_{n \in \mathbb{N}}$ such that

$$
\lim _{l \rightarrow \infty} f\left(x_{n_{l}}\right)=\liminf _{n \rightarrow \infty} f\left(x_{n}\right) \leq f(x) \quad(x \in X) .
$$

Since $\left(x_{n_{l}}\right)_{l \in \mathbb{N}}$ is bounded, there exists $\left(x_{n_{l_{m}}}\right)_{m \in \mathbb{N}}\left(\subset\left(x_{n_{l}}\right)_{l \in \mathbb{N}}\right)$ such that $\left(x_{n_{l_{m}}}\right)_{m \in \mathbb{N}}$ weakly converges to $x_{*} \in H$. Hence, (A6) and (3.6) ensure that $x_{*} \in \operatorname{Fix}\left(Q^{(i)}\right)(i \in \mathcal{I})$, i.e., $x_{*} \in X$. Furthermore, the continuity and convexity of $f$ (see (A2)) imply that $f$ is weakly lower semicontinuous [3. Theorem 9.1], which means that $f\left(x_{*}\right) \leq \liminf _{m \rightarrow \infty} f\left(x_{n_{l_{m}}}\right)$. Therefore, (3.9) leads to the finding that

$$
f\left(x_{*}\right) \leq \liminf _{m \rightarrow \infty} f\left(x_{n_{l_{m}}}\right)=\lim _{m \rightarrow \infty} f\left(x_{n_{l_{m}}}\right) \leq f(x)(x \in X) \text {, i.e., } x_{*} \in X^{\star} .
$$

Let us take another subsequence $\left(x_{n_{l_{k}}}\right)_{k \in \mathbb{N}}\left(\subset\left(x_{n_{l}}\right)_{l \in \mathbb{N}}\right)$ such that $\left(x_{n_{l_{k}}}\right)_{k \in \mathbb{N}}$ weakly converges to $x_{* *} \in H$. A discussion similar to the one for obtaining $x_{*} \in X^{\star}$ guarantees that $x_{* *} \in X^{\star}$. Here, it is proven that $x_{*}=x_{* *}$. Now, let us assume that $x_{*} \neq x_{* *}$. Then, the existence of $\lim _{n \rightarrow \infty}\left\|x_{n}-x^{\star}\right\|\left(x^{\star} \in X^{\star}\right)$ and Opial's condition [29, Lemma 1] imply that

$$
\begin{aligned}
\lim _{n \rightarrow \infty}\left\|x_{n}-x_{*}\right\| & =\lim _{m \rightarrow \infty}\left\|x_{n_{l_{m}}}-x_{*}\right\|<\lim _{m \rightarrow \infty}\left\|x_{n_{l_{m}}}-x_{* *}\right\| \\
& =\lim _{n \rightarrow \infty}\left\|x_{n}-x_{* *}\right\|=\lim _{k \rightarrow \infty}\left\|x_{n_{l_{k}}}-x_{* *}\right\|<\lim _{k \rightarrow \infty}\left\|x_{n_{l_{k}}}-x_{*}\right\| \\
& =\lim _{n \rightarrow \infty}\left\|x_{n}-x_{*}\right\|,
\end{aligned}
$$

which is a contradiction. Hence, $x_{*}=x_{* *}$. Accordingly, any subsequence of $\left(x_{n_{l}}\right)_{l \in \mathbb{N}}$ converges weakly to $x_{*} \in X^{\star}$; i.e., $\left(x_{n_{l}}\right)_{l \in \mathbb{N}}$ converges weakly to $x_{*} \in X^{\star}$. This means that $x_{*}$ is a weak cluster point of $\left(x_{n}\right)_{n \in \mathbb{N}}$ and belongs to $X^{\star}$. A discussion similar to the one for obtaining $x_{*}=x_{* *}$ guarantees that there is only one weak cluster point of $\left(x_{n}\right)_{n \in \mathbb{N}}$, so we can conclude that, in Case $1,\left(x_{n}\right)_{n \in \mathbb{N}}$ weakly converges to a point in $X^{\star}$.

Case 2: Suppose that $x_{0}^{\star} \in X^{\star}$ and $\left(x_{n_{j}}\right)_{j \in \mathbb{N}}\left(\subset\left(x_{n}\right)_{n \in \mathbb{N}}\right)$ exist such that $\left\|x_{n_{j}}-x_{0}^{\star}\right\|<$ $\left\|x_{n_{j}+1}-x_{0}^{\star}\right\|$ for all $j \in \mathbb{N}$. Then, defining $\Gamma_{n}:=\left\|x_{n}-x_{0}^{\star}\right\|(n \in \mathbb{N})$ implies that $\Gamma_{n_{j}}<\Gamma_{n_{j}+1}$ for all $j \in \mathbb{N}$. Assumption 3.2 and the definition of $x_{n}(n \in \mathbb{N})$ guarantee the boundedness of $\left(x_{n}\right)_{n \in \mathbb{N}}$. Moreover, from the quasi-nonexpansivity of $Q_{\alpha}^{(i)}(i \in \mathcal{I}),\left(Q_{\alpha}^{(i)}\left(x_{n}\right)\right)_{n \in \mathbb{N}}(i \in \mathcal{I})$ is also bounded. Accordingly, Proposition 2.2 ensures that $M_{1}, M_{2}<\infty$. Proposition 2.3 
ensures the existence of $m_{1} \in \mathbb{N}$ such that $\Gamma_{\tau(n)}<\Gamma_{\tau(n)+1}$ for all $n \geq m_{1}$, where $\tau(n)$ is defined as in Proposition 2.3. Lemma 3.1(i) means that, for all $n \geq m_{1}$,

$$
\begin{aligned}
& \sum_{i \in \mathcal{I}} \frac{2 \alpha^{(i)}\left(1-\alpha^{(i)}\right)}{I}\left\|x_{\tau(n)}-Q^{(i)}\left(x_{\tau(n)}\right)\right\|^{2} \\
\leq & \Gamma_{\tau(n)}^{2}-\Gamma_{\tau(n)+1}^{2}+2 M_{1} \lambda_{\tau(n)}^{2}-\frac{2 \lambda_{\tau(n)}}{I} \sum_{i \in \mathcal{I}}\left\langle x_{\tau(n)}-x_{0}^{\star}, g_{\tau(n)}^{(i)}\right\rangle \\
< & \left(2 M_{1} \lambda_{\tau(n)}-\frac{2}{I} \sum_{i \in \mathcal{I}}\left\langle x_{\tau(n)}-x_{0}^{\star}, g_{\tau(n)}^{(i)}\right\rangle\right) \lambda_{\tau(n)} .
\end{aligned}
$$

Hence, the condition $\lim _{n \rightarrow \infty} \tau(n)=\infty$ and (C2) imply that

$$
\lim _{n \rightarrow \infty}\left\|x_{\tau(n)}-Q_{\alpha}^{(i)}\left(x_{\tau(n)}\right)\right\|=\lim _{n \rightarrow \infty}\left\|x_{\tau(n)}-Q^{(i)}\left(x_{\tau(n)}\right)\right\|=0(i \in \mathcal{I}) .
$$

Since (3.8) implies $\left(2 \lambda_{\tau(n)} / I\right) M_{\tau(n)}\left(x_{0}^{\star}\right) \leq \Gamma_{\tau(n)}^{2}-\Gamma_{\tau(n)+1}^{2}<0\left(n \geq m_{1}\right)$ and $\lambda_{\tau(n)}>0$ $\left(n \geq m_{1}\right), M_{\tau(n)}\left(x_{0}^{\star}\right)<0\left(n \geq m_{1}\right)$ holds; i.e., for all $n \geq m_{1}$,

$$
f\left(x_{\tau(n)}\right)-f^{\star}<\left(\sqrt{M_{1}}+M_{2}\right) \sum_{i \in \mathcal{I}}\left\|x_{\tau(n)}-Q_{\alpha}^{(i)}\left(x_{\tau(n)}\right)\right\|+I M_{1} \lambda_{\tau(n)} .
$$

Accordingly, (C2) and (3.10) imply that

$$
\limsup _{n \rightarrow \infty} f\left(x_{\tau(n)}\right) \leq f^{\star} .
$$

Choose a subsequence $\left(x_{\tau\left(n_{k}\right)}\right)_{k \in \mathbb{N}}$ of $\left(x_{\tau(n)}\right)_{n \geq m_{1}}$ arbitrarily. The property of the limit superior of $\left(f\left(x_{\tau(n)}\right)\right)_{n \geq m_{1}}$ and (3.12) guarantee that

$$
\limsup _{k \rightarrow \infty} f\left(x_{\tau\left(n_{k}\right)}\right) \leq \limsup _{n \rightarrow \infty} f\left(x_{\tau(n)}\right) \leq f^{\star} .
$$

The boundedness of $\left(x_{\tau\left(n_{k}\right)}\right)_{k \in \mathbb{N}}$ ensures that there exists $\left(x_{\tau\left(n_{k_{l}}\right)}\right)_{l \in \mathbb{N}}\left(\subset\left(x_{\tau\left(n_{k}\right)}\right)_{k \in \mathbb{N}}\right)$ such that $\left(x_{\tau\left(n_{k_{l}}\right)}\right)_{l \in \mathbb{N}}$ weakly converges to $x_{\star} \in H$. Then, (A6) and (3.10) ensure that $x_{\star} \in X$. Moreover, the weakly lower semicontinuity of $f$ and (3.13) guarantee that

$$
f\left(x_{\star}\right) \leq \liminf _{l \rightarrow \infty} f\left(x_{\tau\left(n_{k_{l}}\right)}\right) \leq \limsup _{l \rightarrow \infty} f\left(x_{\tau\left(n_{k_{l}}\right)}\right) \leq f^{\star} \text {; i.e., } x_{\star} \in X^{\star} .
$$

Therefore, $\left(x_{\tau\left(n_{k_{l}}\right)}\right)_{l \in \mathbb{N}}$ weakly converges to $x_{\star} \in X^{\star}$. From Cases 1 and 2 , there exists a subsequence of $\left(x_{n}\right)_{n \in \mathbb{N}}$ that weakly converges to a point in $X^{\star}$.

Suppose that assumption (i) in Theorem 3.2 holds. Since $f:=\sum_{i \in \mathcal{I}} f^{(i)}$ is strongly convex, $X^{\star}$ consists of one point, denoted by $x^{\star}$. In Case 1 , the strong convexity of $f$ guarantees that there exists $\beta>0$ such that, for all $\alpha \in(0,1)$ and for all $l \in \mathbb{N}$,

$$
\frac{\beta}{2} \alpha(1-\alpha)\left\|x_{n_{l}}-x^{\star}\right\|^{2} \leq \alpha f\left(x_{n_{l}}\right)+(1-\alpha) f^{\star}-f\left(\alpha x_{n_{l}}+(1-\alpha) x^{\star}\right) .
$$

Accordingly, from the existence of $\lim _{n \rightarrow \infty}\left\|x_{n}-x^{\star}\right\|$ and (3.9),

$$
\begin{aligned}
\frac{\beta}{2} \alpha(1-\alpha) \lim _{l \rightarrow \infty}\left\|x_{n_{l}}-x^{\star}\right\|^{2} \leq & \lim _{l \rightarrow \infty}\left(\alpha f\left(x_{n_{l}}\right)+(1-\alpha) f^{\star}\right) \\
& +\limsup _{l \rightarrow \infty}\left(-f\left(\alpha x_{n_{l}}+(1-\alpha) x^{\star}\right)\right) \\
\leq & f^{\star}-\liminf _{l \rightarrow \infty} f\left(\alpha x_{n_{l}}+(1-\alpha) x^{\star}\right),
\end{aligned}
$$


which, together with the weak convergence of $\left(x_{n_{l}}\right)_{l \in \mathbb{N}}$ to $x^{\star}$ and the weakly lower semicontinuity of $f$, implies that

$$
\frac{\beta}{2} \alpha(1-\alpha) \lim _{l \rightarrow \infty}\left\|x_{n_{l}}-x^{\star}\right\|^{2} \leq f^{\star}-f\left(\alpha x^{\star}+(1-\alpha) x^{\star}\right)=0 .
$$

That is, $\left(x_{n_{l}}\right)_{l \in \mathbb{N}}$ strongly converges to $x^{\star}$. Therefore, from [3, Theorem 5.11], the whole sequence $\left(x_{n}\right)_{n \in \mathbb{N}}$ strongly converges to $x^{\star}$.

In Case 2, the strong convexity of $f$ leads to the deduction that, for all $\alpha \in(0,1)$ and for all $l \in \mathbb{N}$,

$$
\begin{aligned}
\frac{\beta}{2} \alpha(1-\alpha) \limsup _{l \rightarrow \infty}\left\|x_{\tau\left(n_{k_{l}}\right)}-x^{\star}\right\|^{2} \leq & \alpha \limsup _{l \rightarrow \infty} f\left(x_{\tau\left(n_{k_{l}}\right)}\right)+(1-\alpha) f^{\star} \\
& -\liminf _{l \rightarrow \infty} f\left(\alpha x_{\tau\left(n_{k_{l}}\right)}+(1-\alpha) x^{\star}\right) .
\end{aligned}
$$

The weak convergence of $\left(x_{\tau\left(n_{k_{l}}\right)}\right)_{l \in \mathbb{N}}$ to $x^{\star}$, the weakly lower semicontinuity of $f$, and (3.13) imply that

$$
\frac{\beta}{2} \alpha(1-\alpha) \limsup _{l \rightarrow \infty}\left\|x_{\tau\left(n_{k_{l}}\right)}-x^{\star}\right\|^{2} \leq f^{\star}-f\left(\alpha x^{\star}+(1-\alpha) x^{\star}\right)=0,
$$

which implies that $\left(x_{\tau\left(n_{k_{l}}\right)}\right)_{l \in \mathbb{N}}$ strongly converges to $x^{\star}$.

When another subsequence $\left(x_{\tau\left(n_{k_{m}}\right)}\right)_{m \in \mathbb{N}}\left(\subset\left(x_{\tau\left(n_{k}\right)}\right)_{k \in \mathbb{N}}\right)$ can be chosen, a discussion similar to the one for showing the weak convergence of $\left(x_{\tau\left(n_{k_{l}}\right)}\right)_{l \in \mathbb{N}}$ to a point in $X^{\star}$ guarantees that $\left(x_{\tau\left(n_{k_{m}}\right)}\right)_{m \in \mathbb{N}}$ also weakly converges to a point in $X^{\star}$. Furthermore, a discussion similar to the one for showing the strong convergence of $\left(x_{\tau\left(n_{k_{l}}\right)}\right)_{l \in \mathbb{N}}$ to $x^{\star}$ ensures that $\left(x_{\tau\left(n_{k_{m}}\right)}\right)_{m \in \mathbb{N}}$ strongly converges to the same $x^{\star}$. Hence, it is guaranteed that $\left(x_{\tau\left(n_{k}\right)}\right)_{k \in \mathbb{N}}$ strongly converges to $x^{\star}$. Since $\left(x_{\tau\left(n_{k}\right)}\right)_{k \in \mathbb{N}}$ is an arbitrary subsequence of $\left(x_{\tau(n)}\right)_{n \geq m_{1}}$, $\left(x_{\tau(n)}\right)_{n \geq m_{1}}$ strongly converges to $x^{\star}$; i.e., $\lim _{n \rightarrow \infty} \Gamma_{\tau(n)}=\lim _{n \rightarrow \infty}\left\|x_{\tau(n)}-x^{\star}\right\|=0$. Accordingly, Proposition 2.3 ensures that

$$
\limsup _{n \rightarrow \infty}\left\|x_{n}-x^{\star}\right\| \leq \limsup _{n \rightarrow \infty} \Gamma_{\tau(n)+1}=0
$$

which implies that, in Case 2, the whole sequence $\left(x_{n}\right)_{n \in \mathbb{N}}$ converges to $x^{\star}$.

Suppose that assumption (ii) in Theorem 3.2 holds. Let $x^{\star} \in X^{\star}$ be the unique solution to Problem 2.1. In Case 1, it is guaranteed that $\left(x_{n}\right)_{n \in \mathbb{N}}$ converges to $x^{\star} \in X^{\star}$. In Case 2 , the convergence of $\left.\left(x_{\tau\left(n_{k_{l}}\right)}\right)\right)_{l \in \mathbb{N}}$ to $x^{\star}$ is guaranteed. A discussion similar to the one for showing the strong convergence of $\left(x_{\tau(n)}\right)_{n \geq m_{1}}$ to $x^{\star}$ (see the above paragraph) ensures that $\left(x_{\tau(n)}\right)_{n \geq m_{1}}$ converges to $x^{\star} \in X^{\star}$. Proposition 2.3 thus leads to the convergence of the whole sequence $\left(x_{n}\right)_{n \in \mathbb{N}}$ to $x^{\star}$. This completes the proof.

\subsection{Convergence rate analysis of Algorithm 3.1 with di- minishing step size}

The following corollary establishes the rate of convergence of Algorithm 3.1 for unconstrained nonsmooth convex optimization.

Corollary 3.1. Consider Problem 2.1 when $Q^{(i)}=\operatorname{Id}(i \in \mathcal{I})$ and suppose that the assumptions in Theorem 3.2 hold. Then, for a large enough $n \in \mathbb{N}$,

$$
f\left(x_{n}\right)-f^{\star} \leq I M_{1} \lambda_{n},
$$


where $M_{1}:=\max _{i \in \mathcal{I}} M_{1}^{(i)^{2}}<\infty$ and $M_{1}^{(i)}(i \in \mathcal{I})$ is defined as in Assumption 3.1,

The larger the number of users $I$, the greater the $M_{1}:=\max _{i \in \mathcal{I}} M_{1}^{(i)^{2}}$. Accordingly, Corollary 3.1 implies that, when the same step size sequence is used, the efficiency of Algorithm 3.1 with $Q^{(i)}=\operatorname{Id}(i \in \mathcal{I})$ may decrease as the number of users $I$ increases.

Proof. In Case 1 in the proof of Theorem 3.2, $\liminf _{n \rightarrow \infty} M_{n}\left(x^{\star}\right) \leq 0$ holds, where $M_{n}(x)$ $(n \in \mathbb{N}, x \in H)$ is defined by (3.7) and $\left\{x^{\star}\right\}=X^{\star}$. Let us prove that there exists $k_{1} \in \mathbb{N}$ such that, for all $n \geq k_{1}, M_{n}\left(x^{\star}\right) \leq 0$. If this assertion does not hold, there exist $\gamma>0$ and a subsequence $\left(M_{n_{j}}\left(x^{\star}\right)\right)_{j \in \mathbb{N}}\left(\subset\left(M_{n}\left(x^{\star}\right)\right)_{n \in \mathbb{N}}\right)$ such that $\gamma<M_{n_{j}}\left(x^{\star}\right)$ for all $j \in \mathbb{N}$. Since Theorem 3.2 implies that $\left(x_{n}\right)_{n \in \mathbb{N}}$ strongly converges to $x^{\star}, 0<\gamma \leq \lim _{j \rightarrow \infty} M_{n_{j}}\left(x^{\star}\right) \leq 0$, which is a contradiction. Hence, $M_{n}\left(x^{\star}\right) \leq 0\left(n \geq k_{1}\right)$. Since $Q^{(i)}=\operatorname{Id}(i \in \mathcal{I})$ implies that $\left\|x_{n}-Q_{\alpha}^{(i)}\left(x_{n}\right)\right\|=0(i \in \mathcal{I}, n \in \mathbb{N})$, we have that, for all $n \geq k_{1}, M_{n}\left(x^{\star}\right)=f\left(x_{n}\right)-f^{\star}-$ $I M_{1} \lambda_{n} \leq 0$.

In Case 2 in the proof of Theorem 3.2, the condition $Q^{(i)}=\operatorname{Id}(i \in \mathcal{I})$ and (3.11) lead to the existence of $k_{2} \in \mathbb{N}$ such that, for all $n \geq k_{2}, f\left(x_{\tau(n)}\right)-f^{\star}<I M_{1} \lambda_{\tau(n)}$. This completes the proof.

The following provides the rate of convergence of Algorithm 3.1 for constrained nonsmooth convex optimization under specific conditions.

Corollary 3.2. Suppose that the assumptions in Theorem 3.2 hold. If there exists $\beta^{(i)}>0$ $(i \in \mathcal{I})$ such that $\alpha^{(i)}>\beta^{(i)^{2}} /\left(\beta^{(i)^{2}}+2\right)$ and $\mathrm{d}\left(x_{n}, X\right):=\left\|x_{n}-P_{X}\left(x_{n}\right)\right\| \leq \beta^{(i)} \| x_{n}-$ $Q_{\alpha}^{(i)}\left(x_{n}\right) \|(i \in \mathcal{I}, n \in \mathbb{N})$ and if $\left(\left\|x_{n}-Q^{(i)}\left(x_{n}\right)\right\|\right)_{n \in \mathbb{N}}(i \in \mathcal{I})$ is monotone decreasing, then, for all $i \in \mathcal{I}$ and for all $n \in \mathbb{N}$,

$$
\left\|x_{n}-Q^{(i)}\left(x_{n}\right)\right\|^{2} \leq \frac{I\left(\mathrm{~d}\left(x_{0}, X\right)^{2}+3 M_{1} \sum_{k=0}^{n} \lambda_{k}^{2}\right)}{\left(1-\alpha^{(i)}\right)\left\{\left(\beta^{(i)^{2}}+2\right) \alpha^{(i)}-\beta^{(i)^{2}}\right\}(n+1)},
$$

where $\left(\lambda_{n}\right)_{n \in \mathbb{N}}$ satisfies $\sum_{n=0}^{\infty} \lambda_{n}^{2}<\infty, M_{1}:=\max _{i \in \mathcal{I}} M_{1}^{(i)^{2}}<\infty$, and $M_{1}^{(i)}(i \in \mathcal{I})$ is defined as in Assumption [3.1. Moreover, for a large enough $n \in \mathbb{N}$,

$$
f\left(x_{n}\right)-f^{\star} \leq I\left\{\left(\sqrt{M_{1}}+M_{2}\right) \sqrt{\frac{I M_{3}}{n+1}}+M_{1} \lambda_{n}\right\},
$$

where $M_{2}:=\max _{i \in \mathcal{I}} M_{2}^{(i)}<\infty, M_{2}^{(i)}(i \in \mathcal{I})$ is defined as in Assumption 3.1, $M_{3}:=$ $\max _{i \in \mathcal{I}} M_{3}^{(i)}<\infty$, and $M_{3}^{(i)}:=\left(\mathrm{d}\left(x_{0}, X\right)^{2}+3 M_{1} \sum_{k=0}^{\infty} \lambda_{k}^{2}\right) /\left(\left(1-\alpha^{(i)}\right)\left\{\left(\beta^{(i)^{2}}+2\right) \alpha^{(i)}-\beta^{(i)^{2}}\right\}\right)$ $(i \in \mathcal{I})$.

Consider the case where $\alpha^{(i)}:=1 / 2$ and $Q^{(i)}:=\left(1 /\left(1-\alpha^{(i)}\right)\right)\left(P_{X}-\alpha^{(i)} \mathrm{Id}\right)(i \in$ $\mathcal{I})$; i.e., $Q_{\alpha}^{(i)}=P_{X}(i \in \mathcal{I})$. Then, $Q^{(i)}(i \in \mathcal{I})$ is nonexpansive [3, Proposition 4.25]. Moreover, $\beta^{(i)}=1(i \in \mathcal{I})$ can be chosen such that $\alpha^{(i)}=1 / 2>\beta^{(i)^{2}} /\left(\beta^{(i) 2}+2\right)=1 / 3$ and $\mathrm{d}\left(x_{n}, X\right)=\beta^{(i)}\left\|x_{n}-Q_{\alpha}^{(i)}\left(x_{n}\right)\right\|(i \in \mathcal{I}, n \in \mathbb{N})$. Corollary 3.2 thus implies that, if $\left(\left\|x_{n}-P_{X}\left(x_{n}\right)\right\|\right)_{n \in \mathbb{N}}$ is monotone decreasing, Algorithm 3.1 with $\lambda_{n}:=1 /(n+1)(n \in \mathbb{N})$ satisfies $f\left(x_{n}\right)-f^{\star} \leq I\left\{\left(\sqrt{M_{1}}+M_{2}\right) \sqrt{I M_{3} /(n+1)}+M_{1} /(n+1)\right\}$.

The rate of convergence of Algorithm 3.1 depends on the number of users $I$ and the step size sequence $\left(\lambda_{n}\right)_{n \in \mathbb{N}}$. Since the larger the $I$, the greater the $M_{1}:=\max _{i \in \mathcal{I}} M_{1}^{(i)^{2}}$ and the $M_{2}:=\max _{i \in \mathcal{I}} M_{2}^{(i)}$, Corollary 3.2 implies that, when the same step size sequence is used, 
the efficiency of Algorithm 3.1 may decrease as the number of users $I$ increases, as seen in Corollary 3.1. Section 5 presents examples such that $\left(\left\|x_{n}-Q^{(i)}\left(x_{n}\right)\right\|\right)_{n \in \mathbb{N}}$ generated by Algorithm 3.1 is monotone decreasing.

Proof. Set $z_{n}:=P_{X}\left(x_{n}\right)(n \in \mathbb{N})$. Then, $\mathrm{d}\left(x_{n+1}, X\right):=\inf _{y \in X}\left\|x_{n+1}-y\right\| \leq\left\|x_{n+1}-z_{n}\right\|$ $(n \in \mathbb{N})$. Accordingly, Lemma $3.1(\mathrm{i})$ guarantees that, for all $n \in \mathbb{N}$,

$$
\begin{aligned}
\mathrm{d}\left(x_{n+1}, X\right)^{2} \leq & \mathrm{d}\left(x_{n}, X\right)^{2}-\frac{2}{I} \sum_{i \in \mathcal{I}} \alpha^{(i)}\left(1-\alpha^{(i)}\right)\left\|x_{n}-Q^{(i)}\left(x_{n}\right)\right\|^{2}+2 M_{1} \lambda_{n}^{2} \\
& +\frac{2 \lambda_{n}}{I} \sum_{i \in \mathcal{I}}\left\langle z_{n}-x_{n}, g_{n}^{(i)}\right\rangle,
\end{aligned}
$$

which implies that, for all $N \in \mathbb{N}$,

$$
\begin{aligned}
& \frac{2}{I} \sum_{n=0}^{N} \sum_{i \in \mathcal{I}} \alpha^{(i)}\left(1-\alpha^{(i)}\right)\left\|x_{n}-Q^{(i)}\left(x_{n}\right)\right\|^{2} \\
& \quad \leq \mathrm{d}\left(x_{0}, X\right)^{2}-\mathrm{d}\left(x_{N+1}, X\right)^{2}+2 M_{1} \sum_{n=0}^{N} \lambda_{n}^{2}+\frac{2}{I} \sum_{n=0}^{N} \lambda_{n} \sum_{i \in \mathcal{I}}\left\langle z_{n}-x_{n}, g_{n}^{(i)}\right\rangle .
\end{aligned}
$$

From $2\|x\|\|y\| \leq\|x\|^{2}+\|y\|^{2}(x, y \in H)$ and the Cauchy-Schwarz inequality, $(2 / I) \sum_{n=0}^{N} \sum_{i \in \mathcal{I}}\left\langle z_{n}-\right.$ $\left.x_{n}, \lambda_{n} g_{n}^{(i)}\right\rangle \leq(1 / I) \sum_{n=0}^{N} \sum_{i \in \mathcal{I}}\left(\left\|z_{n}-x_{n}\right\|^{2}+\lambda_{n}^{2}\left\|g_{n}^{(i)}\right\|^{2}\right)$, which, together with the definition of $M_{1}$ and $\left\|x_{n}-z_{n}\right\| \leq \beta^{(i)}\left\|x_{n}-Q_{\alpha}^{(i)}\left(x_{n}\right)\right\|(i \in \mathcal{I}, n \in \mathbb{N})$, implies that

$$
\frac{2}{I} \sum_{n=0}^{N} \sum_{i \in \mathcal{I}}\left\langle z_{n}-x_{n}, \lambda_{n} g_{n}^{(i)}\right\rangle \leq \frac{1}{I} \sum_{n=0}^{N} \sum_{i \in \mathcal{I}} \beta^{(i)^{2}}\left\|x_{n}-Q_{\alpha}^{(i)}\left(x_{n}\right)\right\|^{2}+M_{1} \sum_{n=0}^{N} \lambda_{n}^{2} .
$$

Accordingly, for all $N \in \mathbb{N}$,

$$
\begin{aligned}
& \frac{1}{I} \sum_{n=0}^{N} \sum_{i \in \mathcal{I}}\left(1-\alpha^{(i)}\right)\left\{\left(\beta^{(i)^{2}}+2\right) \alpha^{(i)}-\beta^{(i)^{2}}\right\}\left\|x_{n}-Q^{(i)}\left(x_{n}\right)\right\|^{2} \\
& \quad \leq \mathrm{d}\left(x_{0}, X\right)^{2}+3 M_{1} \sum_{n=0}^{N} \lambda_{n}^{2} .
\end{aligned}
$$

From the monotone decreasing property of $\left(\left\|x_{n}-Q^{(i)}\left(x_{n}\right)\right\|\right)_{n \in \mathbb{N}}(i \in \mathcal{I})$, for all $j \in \mathcal{I}$ and for all $N \in \mathbb{N}$,

$$
\begin{aligned}
& \frac{(N+1)}{I}\left(1-\alpha^{(j)}\right)\left\{\left(\beta^{(j)^{2}}+2\right) \alpha^{(j)}-\beta^{(j)^{2}}\right\}\left\|x_{N}-Q^{(j)}\left(x_{N}\right)\right\|^{2} \\
\leq & \frac{(N+1)}{I} \sum_{i \in \mathcal{I}}\left(1-\alpha^{(i)}\right)\left\{\left(\beta^{(i)^{2}}+2\right) \alpha^{(i)}-\beta^{(i)^{2}}\right\}\left\|x_{N}-Q^{(i)}\left(x_{N}\right)\right\|^{2} \\
\leq & \mathrm{d}\left(x_{0}, X\right)^{2}+3 M_{1} \sum_{n=0}^{N} \lambda_{n}^{2},
\end{aligned}
$$

which implies that, for all $j \in \mathcal{I}$ and for all $N \in \mathbb{N}$,

$$
\left\|x_{N}-Q^{(j)}\left(x_{N}\right)\right\|^{2} \leq \frac{I\left(\mathrm{~d}\left(x_{0}, X\right)^{2}+3 M_{1} \sum_{n=0}^{N} \lambda_{n}^{2}\right)}{\left(1-\alpha^{(j)}\right)\left\{\left(\beta^{(j)^{2}}+2\right) \alpha^{(j)}-\beta^{(j)^{2}}\right\}(N+1)} .
$$


In Case 1 in the proof of Theorem 3.2 $\liminf _{n \rightarrow \infty} M_{n}\left(x^{\star}\right) \leq 0$, where $\left\{x^{\star}\right\}=X^{\star}$. A discussion similar to the one for obtaining $M_{n}\left(x^{\star}\right) \leq 0\left(n \geq k_{1}\right)$ in the proof of Corollary 3.1 implies that there exists $k_{3} \in \mathbb{N}$ such that, for all $n \geq k_{3}, M_{n}\left(x^{\star}\right)=f\left(x_{n}\right)-f^{\star}-$ $\left(\sqrt{M_{1}}+M_{2}\right) \sum_{i \in \mathcal{I}}\left\|x_{n}-Q_{\alpha}^{(i)}\left(x_{n}\right)\right\|-I M_{1} \lambda_{n} \leq 0$. In Case 2 in the proof of Theorem 3.2. (3.11) leads to the existence of $k_{4} \in \mathbb{N}$ such that, for all $n \geq k_{4}, f\left(x_{\tau(n)}\right)-f^{\star}<$ $\left(\sqrt{M_{1}}+M_{2}\right) \sum_{i \in \mathcal{I}}\left\|x_{\tau(n)}-Q_{\alpha}^{(i)}\left(x_{\tau(n)}\right)\right\|+I M_{1} \lambda_{\tau(n)}$. Accordingly, for a large enough $n \in \mathbb{N}$,

$$
\begin{aligned}
f\left(x_{n}\right)-f^{\star} & \leq\left(\sqrt{M_{1}}+M_{2}\right) \sum_{i \in \mathcal{I}}\left(1-\alpha^{(i)}\right)\left\|x_{n}-Q^{(i)}\left(x_{n}\right)\right\|+I M_{1} \lambda_{n} \\
& \leq\left(\sqrt{M_{1}}+M_{2}\right) \sum_{i \in \mathcal{I}}\left(1-\alpha^{(i)}\right) \sqrt{\frac{I M_{3}}{n+1}}+I M_{1} \lambda_{n} \\
& \leq I\left(\sqrt{M_{1}}+M_{2}\right) \sqrt{\frac{I M_{3}}{n+1}}+I M_{1} \lambda_{n} .
\end{aligned}
$$

This completes the proof.

\section{Incremental Subgradient Method}

The section presents a method for solving Problem 2.1 under the assumption that

(A7) each user can communicate with his/her neighbors,

where user $i$ 's neighbors are users $(i-1)$ and $(i+1)(i \in \mathcal{I})$ and user 0 (resp. user $(I+1)$ ) stands for user $I$ (resp. user 1). This assumption implies that the network considered here is a ring-shaped network in which the users form a circle and pass along messages in cyclic order.

\section{Algorithm 4.1.}

Step 0. User $i(i \in \mathcal{I})$ sets $\alpha^{(i)}$ and $\left(\lambda_{n}\right)_{n \in \mathbb{N}} \subset(0, \infty)$. User 1 sets $x_{0}:=x_{0}^{(0)} \in H$.

Step 1. User $i(i \in \mathcal{I})$ computes $x_{n}^{(i)} \in H$ using

$$
x_{n}^{(i)}:=Q_{\alpha}^{(i)}\left(x_{n}^{(i-1)}\right)-\lambda_{n} g_{n}^{(i)} \text {, where } g_{n}^{(i)} \in \partial f^{(i)}\left(Q_{\alpha}^{(i)}\left(x_{n}^{(i-1)}\right)\right) .
$$

Step 2. User I sets

$$
x_{n+1}=x_{n+1}^{(0)}:=x_{n}^{(I)}
$$

and transmits it to user 1 . The algorithm sets $n:=n+1$ and returns to Step 1.

From (A3) and (A7), user $i(i \in \mathcal{I})$ can compute $x_{n}^{(i)}:=Q_{\alpha}^{(i)}\left(x_{n}^{(i-1)}\right)-\lambda_{n} g_{n}^{(i)}$, where $g_{n}^{(i)} \in \partial f^{(i)}\left(Q_{\alpha}^{(i)}\left(x_{n}^{(i-1)}\right)\right)$, by using information $x_{n}^{(i-1)}$ transmitted from user $(i-1)$ and its own private information.

Now, let us consider the differences between Algorithms 3.1 and 4.1. In Algorithm 3.1. user $i$ computes $x_{n}^{(i)}$ by using $x_{n} \in H, \lambda_{n} \in(0, \infty)$, and its own private information $\partial f^{(i)}$ and $Q_{\alpha}^{(i)}$, and each point $x_{n}^{(i)}$ is broadcast to all users. As a result, all users have $\left(x_{n+1}:=(1 / I) \sum_{i \in \mathcal{I}} x_{n}^{(i)}\right)_{n \in \mathbb{N}}$, which strongly converges to a point in $X^{\star}$ (Theorem 3.2). In Algorithm 4.1, user $i$ computes $x_{n}^{(i)}$ by using $\lambda_{n} \in(0, \infty), \partial f^{(i)}, Q_{\alpha}^{(i)}$, and the point $x_{n}^{(i-1)}$ transmitted from user $(i-1)$, and point $x_{n}^{(i)}$ is transmitted to user $(i+1)$. User $i$ in Algorithm 4.1 has $\left(x_{n}^{(i)}\right)_{n \in \mathbb{N}}$, which strongly converges to a point in $X^{\star}$ (Theorem 4.2).

The following assumptions are made here. 
Assumption 4.1. For all $i \in \mathcal{I}$, there exist $N_{1}^{(i)}, N_{2}^{(i)} \in \mathbb{R}$ such that

$$
\begin{aligned}
& \sup \left\{\|g\|: g \in \partial f^{(i)}\left(Q_{\alpha}^{(i)}\left(x_{n}^{(i-1)}\right)\right), n \in \mathbb{N}\right\} \leq N_{1}^{(i)}, \\
& \sup \left\{\|g\|: g \in \partial f^{(i)}\left(x_{n}\right), n \in \mathbb{N}\right\} \leq N_{2}^{(i)} .
\end{aligned}
$$

Assumption 4.2. The sequence $\left(x_{n}^{(i)}\right)_{n \in \mathbb{N}}(i \in \mathcal{I})$ is bounded.

From a discussion similar to the one for obtaining the relationship between Assumptions 3.1 and 3.2. Assumption 4.2 implies Assumption 4.1. Assumption 4.1 is used to perform a convergence analysis of Algorithm 4.1 with a constant step-size rule (Subsection 4.1) while Assumption 4.2 is used to analyze Algorithm 4.1 with a diminishing step-size rule for the same reason described in Section 3. The same discussion as for (3.1) describing the existence of a simple, bounded, closed convex set $X^{(i)}(i \in \mathcal{I})$ satisfying $X^{(i)} \supset \operatorname{Fix}\left(Q^{(i)}\right)$ leads to

$$
x_{n}^{(i)}:=P^{(i)}\left(Q_{\alpha}^{(i)}\left(x_{n}^{(i-1)}\right)-\lambda_{n} g_{n}^{(i)}\right)
$$

instead of $x_{n}^{(i)}$ for Algorithm 4.1, The boundedness of $X^{(i)}$ guarantees that Assumption 4.2 holds (see also (3.1)).

The following lemma can be shown by referring to the proof of Lemma 3.1

Lemma 4.1. Suppose that $\left(x_{n}^{(i)}\right)_{n \in \mathbb{N}}(i \in \mathcal{I})$ is the sequence generated by Algorithm 4.1 and that Assumptions (A1)-(A4), (A7), and 4.1] hold. The following properties then hold:

(i) For all $n \in \mathbb{N}$ and for all $x \in X$,

$$
\begin{aligned}
\left\|x_{n+1}-x\right\|^{2} \leq & \left\|x_{n}-x\right\|^{2}-2 \sum_{i \in \mathcal{I}} \alpha^{(i)}\left(1-\alpha^{(i)}\right)\left\|x_{n}^{(i-1)}-Q^{(i)}\left(x_{n}^{(i-1)}\right)\right\|^{2} \\
& +2 I N_{1} \lambda_{n}^{2}-2 \lambda_{n} \sum_{i \in \mathcal{I}}\left\langle x_{n}^{(i-1)}-x, g_{n}^{(i)}\right\rangle,
\end{aligned}
$$

where $N_{1}:=\max _{i \in \mathcal{I}} N_{1}^{(i)^{2}}<\infty$.

(ii) For all $n \in \mathbb{N}$ and for all $x \in X$,

$$
\begin{aligned}
\left\|x_{n+1}-x\right\|^{2} \leq & \left\|x_{n}-x\right\|^{2}+2 \lambda_{n}\left(f(x)-f\left(x_{n}\right)\right) \\
& +2 I N_{1} \lambda_{n}^{2}+2 \lambda_{n}\left\{\sqrt{N_{1}} \sum_{i \in \mathcal{I}}\left\|Q_{\alpha}^{(i)}\left(x_{n}^{(i-1)}\right)-x_{n}^{(i-1)}\right\|\right. \\
& \left.+N_{2} \sum_{i \in \mathcal{I}}\left\|Q_{\alpha}^{(i)}\left(x_{n}^{(i-1)}\right)-x_{n}\right\|\right\},
\end{aligned}
$$

where $N_{2}:=\max _{i \in \mathcal{I}} N_{2}^{(i)}<\infty$.

Proof. (i) The sequence $\left(x_{n}^{(i)}\right)_{n \in \mathbb{N}}(i \in \mathcal{I})$ in Algorithm 3.1 is defined by $x_{n}^{(i)}:=Q_{\alpha}^{(i)}\left(x_{n}\right)-$ $\lambda_{n} g_{n}^{(i)}$ while $\left(x_{n}^{(i)}\right)_{n \in \mathbb{N}}(i \in \mathcal{I})$ in Algorithm 4.1 is defined by $x_{n}^{(i)}:=Q_{\alpha}^{(i)}\left(x_{n}^{(i-1)}\right)-\lambda_{n} g_{n}^{(i)}$. Hence, by replacing $x_{n}$ in the proof of Lemma 3.1(i) by $x_{n}^{(i-1)}$, we find that, for all $n \in \mathbb{N}$, for all $i \in \mathcal{I}$, and for all $x \in X$,

$$
\begin{aligned}
\left\|x_{n}^{(i)}-x\right\|^{2} \leq & \left\|x_{n}^{(i-1)}-x\right\|^{2}-2 \alpha^{(i)}\left(1-\alpha^{(i)}\right)\left\|x_{n}^{(i-1)}-Q^{(i)}\left(x_{n}^{(i-1)}\right)\right\|^{2} \\
& +2 N_{1} \lambda_{n}^{2}-2 \lambda_{n}\left\langle x_{n}^{(i-1)}-x, g_{n}^{(i)}\right\rangle
\end{aligned}
$$


where $N_{1}:=\max _{i \in \mathcal{I}} N_{1}^{(i)^{2}}$ and $N_{1}<\infty$ holds from Assumption 4.1 Therefore, for all $n \in \mathbb{N}$ and for all $x \in X$,

$$
\begin{aligned}
\left\|x_{n+1}-x\right\|^{2}= & \left\|x_{n}^{(I)}-x\right\|^{2} \\
\leq & \left\|x_{n}-x\right\|^{2}-2 \sum_{i \in \mathcal{I}} \alpha^{(i)}\left(1-\alpha^{(i)}\right)\left\|x_{n}^{(i-1)}-Q^{(i)}\left(x_{n}^{(i-1)}\right)\right\|^{2} \\
& +2 I N_{1} \lambda_{n}^{2}-2 \lambda_{n} \sum_{i \in \mathcal{I}}\left\langle x_{n}^{(i-1)}-x, g_{n}^{(i)}\right\rangle .
\end{aligned}
$$

(ii) The same discussion as in the proof of Lemma 3.1(ii) implies that, for all $n \in \mathbb{N}$ and for all $x \in X$,

$$
\begin{aligned}
\sum_{i \in \mathcal{I}}\left\langle x-x_{n}^{(i-1)}, g_{n}^{(i)}\right\rangle \leq & f(x)-f\left(x_{n}\right)+\sum_{i \in \mathcal{I}}\left(f^{(i)}\left(x_{n}\right)-f^{(i)}\left(Q_{\alpha}^{(i)}\left(x_{n}^{(i-1)}\right)\right)\right) \\
& +\sqrt{N_{1}} \sum_{i \in \mathcal{I}}\left\|Q_{\alpha}^{(i)}\left(x_{n}^{(i-1)}\right)-x_{n}^{(i-1)}\right\| .
\end{aligned}
$$

Set $N_{2}:=\max _{i \in \mathcal{I}} N_{2}^{(i)}<\infty$. Since $g \in \partial f^{(i)}\left(x_{n}\right)(n \in \mathbb{N})$ implies that, for all $i \in \mathcal{I}$ and for all $n \in \mathbb{N}, f^{(i)}\left(x_{n}\right)-f^{(i)}\left(Q_{\alpha}^{(i)}\left(x_{n}^{(i-1)}\right)\right) \leq\left\langle x_{n}-Q_{\alpha}^{(i)}\left(x_{n}^{(i-1)}\right), g\right\rangle \leq N_{2}\left\|x_{n}-Q_{\alpha}^{(i)}\left(x_{n}^{(i-1)}\right)\right\|$, it is found that

$$
\begin{aligned}
\sum_{i \in \mathcal{I}}\left\langle x-x_{n}, g_{n}^{(i)}\right\rangle \leq & f(x)-f\left(x_{n}\right)+\sqrt{N_{1}} \sum_{i \in \mathcal{I}}\left\|Q_{\alpha}^{(i)}\left(x_{n}^{(i-1)}\right)-x_{n}^{(i-1)}\right\| \\
& +N_{2} \sum_{i \in \mathcal{I}}\left\|Q_{\alpha}^{(i)}\left(x_{n}^{(i-1)}\right)-x_{n}\right\| .
\end{aligned}
$$

Accordingly, Lemma 4.1(i) leads to Lemma 4.1(ii). This completes the proof.

\subsection{Constant step-size rule}

Let us perform a convergence analysis of Algorithm 4.1 with a constant step size.

Theorem 4.1. Suppose that Assumptions (A1)-(A4), (A7), 3.3, and 4.1] hold. Then, $\left(x_{n}\right)_{n \in \mathbb{N}}$ in Algorithm 4.1 satisfies the relations

$$
\begin{aligned}
\liminf _{n \rightarrow \infty} \| x_{n}^{(i-1)}- & Q^{(i)}\left(x_{n}^{(i-1)}\right) \|^{2} \leq \frac{N_{\lambda} \lambda}{\alpha^{(i)}\left(1-\alpha^{(i)}\right)}(i \in \mathcal{I}) \\
\liminf _{n \rightarrow \infty} f\left(x_{n}\right) \leq & f^{\star}+I\left(\frac{I \sqrt{N_{1}} N_{2}}{2}+N_{1}\right) \lambda+N_{2} \sum_{i \in \mathcal{I}} \sum_{j=1}^{i-1} \sqrt{\frac{\left(1-\alpha^{(j)}\right) N_{\lambda} \lambda}{\alpha^{(j)}}} \\
& +\left(\sqrt{N_{1}}+N_{2}\right) \sum_{i \in \mathcal{I}} \sqrt{\frac{\left(1-\alpha^{(j)}\right) N_{\lambda} \lambda}{\alpha^{(i)}}},
\end{aligned}
$$

where $N_{1}$ and $N_{2}$ are as in Lemma 4.1 and, for some $x \in X, N_{\lambda}:=\sup _{n \in \mathbb{N}}\left(I N_{1} \lambda+\right.$ $\left.\left|\sum_{i \in \mathcal{I}}\left\langle x-x_{n}^{(i-1)}, g_{n}^{(i)}\right\rangle\right|\right)$. 
Proof. Choose $x \in X$ arbitrarily and set $N_{\lambda}:=\sup _{n \in \mathbb{N}}\left(I N_{1} \lambda+\left|\sum_{i \in \mathcal{I}}\left\langle x-x_{n}^{(i-1)}, g_{n}^{(i)}\right\rangle\right|\right)$. Since Theorem 4.1 holds when $N_{\lambda}=\infty$, it can be assumed that $N_{\lambda}<\infty$. First, let us show that

$$
\liminf _{n \rightarrow \infty} \sum_{i \in \mathcal{I}} \alpha^{(i)}\left(1-\alpha^{(i)}\right)\left\|x_{n}^{(i-1)}-Q^{(i)}\left(x_{n}^{(i-1)}\right)\right\|^{2} \leq N_{\lambda} \lambda .
$$

Let us assume that (4.2) does not hold. Accordingly, from the same discussion as in the proof of Theorem 3.1. $\delta(>0)$ and $n_{0}(\in \mathbb{N})$ can be chosen such that, for all $n \geq n_{0}$, $\sum_{i \in \mathcal{I}} \alpha^{(i)}\left(1-\alpha^{(i)}\right)\left\|x_{n}^{(i-1)}-Q^{(i)}\left(x_{n}^{(i-1)}\right)\right\|^{2}>N_{\lambda} \lambda+\delta$. Hence, Lemma 4.1(i) leads to the finding that, for all $n \geq n_{0}$,

$$
\begin{aligned}
\left\|x_{n+1}-x\right\|^{2} & <\left\|x_{n}-x\right\|^{2}-2\left\{N_{\lambda} \lambda+\delta\right\}+2 N_{\lambda} \lambda \\
& =\left\|x_{n}-x\right\|^{2}-2 \delta .
\end{aligned}
$$

Therefore, induction shows that $0 \leq\left\|x_{n+1}-x\right\|^{2}<\left\|x_{n_{0}}-x\right\|^{2}-2 \delta\left(n+1-n_{0}\right)\left(n \geq n_{0}\right)$, which is a contradiction. Therefore, (4.2) holds. This means that

$$
\liminf _{n \rightarrow \infty}\left\|x_{n}^{(i-1)}-Q^{(i)}\left(x_{n}^{(i-1)}\right)\right\|^{2} \leq \frac{N_{\lambda} \lambda}{\alpha^{(i)}\left(1-\alpha^{(i)}\right)}(i \in \mathcal{I}) .
$$

Let $i \in \mathcal{I}$ be fixed arbitrarily. Inequality (4.3) and the property of the limit inferior of $\left(\left\|x_{n}^{(i)}-Q^{(i)}\left(x_{n}^{(i)}\right)\right\|^{2}\right)_{n \in \mathbb{N}}$ guarantee the existence of a subsequence $\left(x_{n_{k}}^{(i)}\right)_{k \in \mathbb{N}}$ of $\left(x_{n}^{(i)}\right)_{n \in \mathbb{N}}$ such that $\lim _{k \rightarrow \infty}\left\|x_{n_{k}}^{(i-1)}-Q^{(i)}\left(x_{n_{k}}^{(i-1)}\right)\right\|^{2}=\liminf _{n \rightarrow \infty}\left\|x_{n}^{(i-1)}-Q^{(i)}\left(x_{n}^{(i-1)}\right)\right\|^{2} \leq N_{\lambda} \lambda /\left(\alpha^{(i)}(1-\right.$ $\left.\left.\alpha^{(i)}\right)\right)$. Therefore, for all $\epsilon>0$, there exists $k_{0} \in \mathbb{N}$ such that, for all $k \geq k_{0}$,

$$
\left\|x_{n_{k}}^{(i-1)}-Q^{(i)}\left(x_{n_{k}}^{(i-1)}\right)\right\| \leq \sqrt{\frac{N_{\lambda} \lambda}{\alpha^{(i)}\left(1-\alpha^{(i)}\right)}+\epsilon} .
$$

Now, let us prove that, for all $k \geq k_{0}$,

$$
\begin{aligned}
\liminf _{n \rightarrow \infty} f\left(x_{n}\right) \leq & f^{\star}+I N_{1} \lambda+\sqrt{N_{1}} \sum_{i \in \mathcal{I}}\left\|x_{n_{k}}^{(i-1)}-Q_{\alpha}^{(i)}\left(x_{n_{k}}^{(i-1)}\right)\right\| \\
& +N_{2} \sum_{i \in \mathcal{I}}\left\|x_{n_{k}}-Q_{\alpha}^{(i)}\left(x_{n_{k}}^{(i-1)}\right)\right\|+2 \epsilon .
\end{aligned}
$$

Let us assume that (4.5) does not hold for all $k \geq k_{0}$. Then, (A5) and the property of the limit inferior of $\left(f\left(x_{n}\right)\right)_{n \in \mathbb{N}}$ guarantee that $x^{\star} \in X^{\star}$ and $n_{1} \in \mathbb{N}$ exist such that, for all $n \geq n_{1}$,

$$
\begin{aligned}
f\left(x_{n}\right)-f\left(x^{\star}\right)> & I N_{1} \lambda+\sqrt{N_{1}} \sum_{i \in \mathcal{I}}\left\|x_{n}^{(i-1)}-Q_{\alpha}^{(i)}\left(x_{n}^{(i-1)}\right)\right\| \\
& +N_{2} \sum_{i \in \mathcal{I}}\left\|x_{n}-Q_{\alpha}^{(i)}\left(x_{n}^{(i-1)}\right)\right\|+\epsilon .
\end{aligned}
$$


Therefore, Lemma 4.1(ii) means that, for all $n \geq n_{1}$,

$$
\begin{aligned}
& \left\|x_{n+1}-x^{\star}\right\|^{2} \\
\leq & \left\|x_{n}-x^{\star}\right\|^{2}+2 I N_{1} \lambda^{2}+2 \lambda\left(f\left(x^{\star}\right)-f\left(x_{n}\right)\right) \\
& +2 \lambda\left\{\sqrt{N_{1}} \sum_{i \in \mathcal{I}}\left\|x_{n}^{(i-1)}-Q_{\alpha}^{(i)}\left(x_{n}^{(i-1)}\right)\right\|+N_{2} \sum_{i \in \mathcal{I}}\left\|x_{n}-Q_{\alpha}^{(i)}\left(x_{n}^{(i-1)}\right)\right\|\right\} \\
< & \left\|x_{n}-x^{\star}\right\|^{2}+2 I N_{1} \lambda^{2} \\
& -2 \lambda\left\{I N_{1} \lambda+\sqrt{N_{1}} \sum_{i \in \mathcal{I}}\left\|x_{n}^{(i-1)}-Q_{\alpha}^{(i)}\left(x_{n}^{(i-1)}\right)\right\|\right. \\
& \left.+N_{2} \sum_{i \in \mathcal{I}}\left\|x_{n}-Q_{\alpha}^{(i)}\left(x_{n}^{(i-1)}\right)\right\|+\epsilon\right\}+2 \lambda\left\{\sqrt{N_{1}} \sum_{i \in \mathcal{I}}\left\|x_{n}^{(i-1)}-Q_{\alpha}^{(i)}\left(x_{n}^{(i-1)}\right)\right\|\right. \\
& \left.+N_{2} \sum_{i \in \mathcal{I}}\left\|x_{n}-Q_{\alpha}^{(i)}\left(x_{n}^{(i-1)}\right)\right\|\right\} \\
= & \left\|x_{n}-x^{\star}\right\|^{2}-2 \lambda \epsilon,
\end{aligned}
$$

which means that $0 \leq\left\|x_{n+1}-x^{\star}\right\|^{2}<\left\|x_{n_{1}}-x^{\star}\right\|^{2}-2 \lambda \epsilon\left(n+1-n_{1}\right)\left(n \geq n_{1}\right)$. Hence, there is a contradiction. Accordingly, (4.5) holds for all $k \geq k_{0}$.

Furthermore, the triangle inequality implies that, for all $k \geq k_{0}$,

$$
\begin{aligned}
& \sqrt{N_{1}} \sum_{i \in \mathcal{I}}\left\|x_{n_{k}}^{(i-1)}-Q_{\alpha}^{(i)}\left(x_{n_{k}}^{(i-1)}\right)\right\|+N_{2} \sum_{i \in \mathcal{I}}\left\|x_{n_{k}}-Q_{\alpha}^{(i)}\left(x_{n_{k}}^{(i-1)}\right)\right\| \\
\leq & \sqrt{N_{1}} \sum_{i \in \mathcal{I}}\left\|x_{n_{k}}^{(i-1)}-Q_{\alpha}^{(i)}\left(x_{n_{k}}^{(i-1)}\right)\right\|+N_{2} \sum_{i \in \mathcal{I}}\left\|x_{n_{k}}^{(0)}-x_{n_{k}}^{(i-1)}\right\| \\
& +N_{2} \sum_{i \in \mathcal{I}}\left\|x_{n_{k}}^{(i-1)}-Q_{\alpha}^{(i)}\left(x_{n_{k}}^{(i-1)}\right)\right\| \\
\leq & \left(\sqrt{N_{1}}+N_{2}\right) \sum_{i \in \mathcal{I}}\left(1-\alpha^{(i)}\right)\left\|x_{n_{k}}^{(i-1)}-Q^{(i)}\left(x_{n_{k}}^{(i-1)}\right)\right\| \\
& +N_{2} \sum_{i \in \mathcal{I}} \sum_{j=1}^{i-1}\left\|x_{n_{k}}^{(j-1)}-x_{n_{k}}^{(j)}\right\| .
\end{aligned}
$$

Moreover, the definition of $x_{n}^{(i)}(n \in \mathbb{N}, i \in \mathcal{I})$ and the triangle inequality mean that, for all $k \geq k_{0}$,

$$
\begin{aligned}
\sum_{i \in \mathcal{I}} \sum_{j=1}^{i-1}\left\|x_{n_{k}}^{(j-1)}-x_{n_{k}}^{(j)}\right\| & \leq \sum_{i \in \mathcal{I}} \sum_{j=1}^{i-1}\left\|x_{n_{k}}^{(j-1)}-Q_{\alpha}^{(j)}\left(x_{n_{k}}^{(j-1)}\right)\right\|+\sum_{i \in \mathcal{I}} \sum_{j=1}^{i-1} \sqrt{N_{1}} \lambda \\
& =\sum_{i \in \mathcal{I}} \sum_{j=1}^{i-1}\left\|x_{n_{k}}^{(j-1)}-Q_{\alpha}^{(j)}\left(x_{n_{k}}^{(j-1)}\right)\right\|+\frac{I^{2} \sqrt{N_{1}} \lambda}{2} .
\end{aligned}
$$


Accordingly, (4.4) guarantees that

$$
\begin{aligned}
& \sqrt{N_{1}} \sum_{i \in \mathcal{I}}\left\|x_{n_{k}}^{(i-1)}-Q_{\alpha}^{(i)}\left(x_{n_{k}}^{(i-1)}\right)\right\|+N_{2} \sum_{i \in \mathcal{I}}\left\|x_{n_{k}}-Q_{\alpha}^{(i)}\left(x_{n_{k}}^{(i-1)}\right)\right\| \\
\leq & \left(\sqrt{N_{1}}+N_{2}\right) \sum_{i \in \mathcal{I}}\left(1-\alpha^{(i)}\right) \sqrt{\frac{N_{\lambda} \lambda}{\alpha^{(i)}\left(1-\alpha^{(i)}\right)}}+\epsilon \\
& +N_{2} \sum_{i \in \mathcal{I}} \sum_{j=1}^{i-1}\left(1-\alpha^{(j)}\right) \sqrt{\frac{N_{\lambda} \lambda}{\alpha^{(j)}\left(1-\alpha^{(j)}\right)}+\epsilon}+\frac{I^{2} \sqrt{N_{1}} N_{2} \lambda}{2} .
\end{aligned}
$$

Therefore, (4.5) leads to the finding that, for all $\epsilon>0$,

$$
\begin{aligned}
\liminf _{n \rightarrow \infty} f\left(x_{n}\right) \leq & f^{\star}+I\left(\frac{I \sqrt{N_{1}} N_{2}}{2}+N_{1}\right) \lambda \\
& +\left(\sqrt{N_{1}}+N_{2}\right) \sum_{i \in \mathcal{I}}\left(1-\alpha^{(i)}\right) \sqrt{\frac{N_{\lambda} \lambda}{\alpha^{(i)}\left(1-\alpha^{(i)}\right)}+\epsilon} \\
& +N_{2} \sum_{i \in \mathcal{I}} \sum_{j=1}^{i-1}\left(1-\alpha^{(j)}\right) \sqrt{\frac{N_{\lambda} \lambda}{\alpha^{(j)}\left(1-\alpha^{(j)}\right)}+\epsilon}+2 \epsilon .
\end{aligned}
$$

Hence, the arbitrary property of $\epsilon(>0)$ leads to the deduction that

$$
\begin{aligned}
& \liminf _{n \rightarrow \infty} f\left(x_{n}\right) \\
& \leq f^{\star}+I\left(\frac{I \sqrt{N_{1}} N_{2}}{2}+N_{1}\right) \lambda+N_{2} \sum_{i \in \mathcal{I}} \sum_{j=1}^{i-1}\left(1-\alpha^{(j)}\right) \sqrt{\frac{N_{\lambda} \lambda}{\alpha^{(j)}\left(1-\alpha^{(j)}\right)}} \\
& \quad+\left(\sqrt{N_{1}}+N_{2}\right) \sum_{i \in \mathcal{I}}\left(1-\alpha^{(i)}\right) \sqrt{\frac{N_{\lambda} \lambda}{\alpha^{(i)}\left(1-\alpha^{(i)}\right)}} \\
& =f^{\star}+I\left(\frac{I \sqrt{N_{1}} N_{2}}{2}+N_{1}\right) \lambda \\
& \quad+\left(\sqrt{N_{1}}+N_{2}\right) \sum_{i \in \mathcal{I}} \sqrt{\frac{\left(1-\alpha^{(j)}\right) N_{\lambda} \lambda}{\alpha^{(i)}}}+N_{2} \sum_{i \in \mathcal{I}} \sum_{j=1}^{i-1} \sqrt{\frac{\left(1-\alpha^{(j)}\right) N_{\lambda} \lambda}{\alpha^{(j)}}} .
\end{aligned}
$$

This completes the proof.

\subsection{Diminishing step-size rule}

Let us perform a convergence analysis of Algorithm 4.1 with a diminishing step size.

Theorem 4.2. Suppose that Assumptions (A1)-(A4), (A7), 3.4, and 4.2 hold. Then there exists a subsequence of $\left(x_{n}^{(i)}\right)_{n \in \mathbb{N}}(i \in \mathcal{I})$ generated by Algorithm 4.1 that weakly converges to a point in $X^{\star}$. If either (i) or (ii) in Theorem 3.2 holds, $\left(x_{n}^{(i)}\right)_{n \in \mathbb{N}}(i \in \mathcal{I})$ strongly converges to a unique point in $X^{\star} 4$

\footnotetext{
${ }^{4}$ Figure 10 shows the existence of a subsequence of $\left(x_{n}\right)_{n \in \mathbb{N}}$ generated by Algorithm 4.1 that converges to a solution to Problem 5.1 when all $f^{(i)}$ are convex while Figure 12 indicates the convergence of $\left(x_{n}\right)_{n \in \mathbb{N}}$ generated by Algorithm 4.1 to the solution to Problem 5.1 when only $f^{(1)}$ is strongly convex.
} 
Proof. Case 1: Suppose there exists $m_{0} \in \mathbb{N}$ such that $\left\|x_{n+1}-x^{\star}\right\| \leq\left\|x_{n}-x^{\star}\right\|$ for all $n \geq m_{0}$ and for all $x^{\star} \in X^{\star}$. Then, there exists $\lim _{n \rightarrow \infty}\left\|x_{n}-x^{\star}\right\|$ for all $x^{\star} \in X^{\star}$. Hence, $\left(x_{n}\right)_{n \in \mathbb{N}}$ is bounded. From the quasi-nonexpansivity of $Q_{\alpha}^{(1)},\left(Q_{\alpha}^{(1)}\left(x_{n}\right)\right)_{n \in \mathbb{N}}$ is also bounded. Hence, Proposition 2.2 guarantees the boundedness of $\left(g_{n}^{(1)}\right)_{n \in \mathbb{N}}$. Inequality (4.1) when $i=1, x_{n}^{(0)}:=x_{n}(n \in \mathbb{N})$, and $(\mathrm{C} 2)$ lead to the boundedness of $\left(x_{n}^{(1)}\right)_{n \in \mathbb{N}}$. Therefore, induction shows that $\left(x_{n}^{(i)}\right)_{n \in \mathbb{N}}$ and $\left(g_{n}^{(i)}\right)_{n \in \mathbb{N}}(i \in \mathcal{I})$ are bounded; i.e., $N_{1}$ and $N_{2}$ defined as in Lemma 4.1] are finite. Lemma 4.1(i) implies that, for all $n \geq m_{0}$ and for all $x^{\star} \in X^{\star}$,

$$
\begin{aligned}
& 2 \sum_{i \in \mathcal{I}} \alpha^{(i)}\left(1-\alpha^{(i)}\right)\left\|x_{n}^{(i-1)}-Q^{(i)}\left(x_{n}^{(i-1)}\right)\right\|^{2} \\
\leq & \left\|x_{n}-x^{\star}\right\|^{2}-\left\|x_{n+1}-x^{\star}\right\|^{2}+2 I N_{1} \lambda_{n}^{2}-2 \lambda_{n} \sum_{i \in \mathcal{I}}\left\langle x_{n}^{(i-1)}-x^{\star}, g_{n}^{(i)}\right\rangle .
\end{aligned}
$$

Accordingly, the existence of $\lim _{n \rightarrow \infty}\left\|x_{n}-x^{\star}\right\|\left(x^{\star} \in X^{\star}\right)$ and (C2) guarantee that

$$
\lim _{n \rightarrow \infty}\left\|x_{n}^{(i-1)}-Q_{\alpha}^{(i)}\left(x_{n}^{(i-1)}\right)\right\|=\lim _{n \rightarrow \infty}\left\|x_{n}^{(i-1)}-Q^{(i)}\left(x_{n}^{(i-1)}\right)\right\|=0(i \in \mathcal{I}) .
$$

Moreover, since $\left\|x_{n}^{(i-1)}-x_{n}^{(i)}\right\|=\left\|x_{n}^{(i-1)}-Q_{\alpha}^{(i)}\left(x_{n}^{(i-1)}\right)+\lambda_{n} g_{n}^{(i)}\right\| \leq\left\|x_{n}^{(i-1)}-Q_{\alpha}^{(i)}\left(x_{n}^{(i-1)}\right)\right\|+$ $\sqrt{N_{1}} \lambda_{n}(n \in \mathbb{N}, i \in \mathcal{I})$, (4.6) and (C2) ensure that $\lim _{n \rightarrow \infty}\left\|x_{n}^{(i-1)}-x_{n}^{(i)}\right\|=0(i \in \mathcal{I})$. Since the triangle inequality implies that $\left\|x_{n}-x_{n}^{(i-1)}\right\| \leq \sum_{j=1}^{i-1}\left\|x_{n}^{(j-1)}-x_{n}^{(j)}\right\|(n \in \mathbb{N}, i \in \mathcal{I})$,

$$
\lim _{n \rightarrow \infty}\left\|x_{n}-x_{n}^{(i-1)}\right\|=0(i \in \mathcal{I})
$$

From $\left\|x_{n}-Q_{\alpha}^{(i)}\left(x_{n}^{(i-1)}\right)\right\| \leq\left\|x_{n}-x_{n}^{(i-1)}\right\|+\left\|x_{n}^{(i-1)}-Q_{\alpha}^{(i)}\left(x_{n}^{(i-1)}\right)\right\|(n \in \mathbb{N}, i \in \mathcal{I})$,

$$
\lim _{n \rightarrow \infty}\left\|x_{n}-Q_{\alpha}^{(i)}\left(x_{n}^{(i-1)}\right)\right\|=0(i \in \mathcal{I}) .
$$

Here, let us define that, for all $n \in \mathbb{N}$ and for all $x \in X$,

$$
\begin{aligned}
N_{n}(x):= & f\left(x_{n}\right)-f(x)-\sqrt{N_{1}} \sum_{i \in \mathcal{I}}\left\|Q_{\alpha}^{(i)}\left(x_{n}^{(i-1)}\right)-x_{n}^{(i-1)}\right\| \\
& -N_{2} \sum_{i \in \mathcal{I}}\left\|Q_{\alpha}^{(i)}\left(x_{n}^{(i-1)}\right)-x_{n}\right\|-I N_{1} \lambda_{n} .
\end{aligned}
$$

Then, Lemma 4.1(ii) leads to the finding that, for all $n \in \mathbb{N}$ and for all $x \in X$,

$$
2 \lambda_{n} N_{n}(x) \leq\left\|x_{n}-x\right\|^{2}-\left\|x_{n+1}-x\right\|^{2} .
$$

A discussion similar to the one for obtaining $\liminf _{n \rightarrow \infty} M_{n}(x) \leq 0(x \in X)$ guarantees that $\liminf _{n \rightarrow \infty} N_{n}(x) \leq 0(x \in X)$, which, together with (C2), (4.6), and (4.8), implies that $\liminf _{n \rightarrow \infty} f\left(x_{n}\right) \leq f(x)(x \in X)$. Accordingly, there exists a subsequence $\left(x_{n_{l}}\right)_{l \in \mathbb{N}}$ of $\left(x_{n}\right)_{n \in \mathbb{N}}$ such that $\lim _{l \rightarrow \infty} f\left(x_{n_{l}}\right)=\liminf _{n \rightarrow \infty} f\left(x_{n}\right) \leq f(x)(x \in X)$. Since $\left(x_{n_{l}}\right)_{l \in \mathbb{N}}$ is bounded, there exists $\left(x_{n_{l_{m}}}\right)_{m \in \mathbb{N}}\left(\subset\left(x_{n_{l}}\right)_{l \in \mathbb{N}}\right)$ such that $\left(x_{n_{l_{m}}}\right)_{m \in \mathbb{N}}$ weakly converges to $x_{*} \in H$. Equation (4.7) guarantees that $\left(x_{n_{l_{m}}}^{(i-1)}\right)_{m \in \mathbb{N}}(i \in \mathcal{I})$ weakly converges to $x_{*}$. Thus, (A6) and (4.6) ensure that $x_{*} \in X$. From the same discussion as in the proof of Theorem 3.2 $\left(x_{n}\right)_{n \in \mathbb{N}}$ weakly converges to a point in $X^{\star}$. Moreover, (4.7) implies that $\left(x_{n}^{(i)}\right)_{n \in \mathbb{N}}(i \in \mathcal{I})$ weakly converges to a point in $X^{\star}$. 
Case 2: Suppose that $x_{0}^{*} \in X^{\star}$ and $\left(x_{n_{j}}\right)_{j \in \mathbb{N}}\left(\subset\left(x_{n}\right)_{n \in \mathbb{N}}\right)$ exist such that $\Gamma_{n_{j}}:=$ $\left\|x_{n_{j}}-x_{0}^{*}\right\|<\left\|x_{n_{j}+1}-x_{0}^{*}\right\|$ for all $j \in \mathbb{N}$. Assumption 4.2 and the quasi-nonexpansivity of $Q_{\alpha}^{(i)}(i \in \mathcal{I})$ guarantee the boundedness of $\left(Q_{\alpha}^{(i)}\left(x_{n}^{(i-1)}\right)\right)_{n \in \mathbb{N}}(i \in \mathcal{I})$. Hence, Proposition 2.2 ensures that $N_{1}, N_{2}<\infty$. Proposition 2.3 means the existence of $m_{1} \in \mathbb{N}$ such that $\Gamma_{\tau(n)}<\Gamma_{\tau(n)+1}$ for all $n \geq m_{1}$, where $\tau(n)$ is as in Proposition 2.3. Lemma 4.1(i) means that, for all $n \geq m_{1}$,

$$
\begin{aligned}
& 2 \sum_{i \in \mathcal{I}} \alpha^{(i)}\left(1-\alpha^{(i)}\right)\left\|x_{\tau(n)}^{(i-1)}-Q^{(i)}\left(x_{\tau(n)}^{(i-1)}\right)\right\|^{2} \\
\leq & \Gamma_{\tau(n)}^{2}-\Gamma_{\tau(n)+1}^{2}+2 I N_{1} \lambda_{\tau(n)}^{2}-2 \lambda_{\tau(n)} \sum_{i \in \mathcal{I}}\left\langle x_{\tau(n)}^{(i-1)}-x_{0}^{*}, g_{\tau(n)}^{(i)}\right\rangle \\
< & \left(2 I N_{1} \lambda_{\tau(n)}-2 \sum_{i \in \mathcal{I}}\left\langle x_{\tau(n)}^{(i-1)}-x_{0}^{*}, g_{\tau(n)}^{(i)}\right\rangle\right) \lambda_{\tau(n)},
\end{aligned}
$$

which, together with $\lim _{n \rightarrow \infty} \tau(n)=\infty$ and (C2), implies that

$$
\lim _{n \rightarrow \infty}\left\|x_{\tau(n)}^{(i-1)}-Q^{(i)}\left(x_{\tau(n)}^{(i-1)}\right)\right\|=0(i \in \mathcal{I}) .
$$

The same discussions for obtaining (4.6), (4.7), and (4.8) imply that

$$
\begin{aligned}
& \lim _{n \rightarrow \infty}\left\|x_{\tau(n)}^{(i-1)}-Q_{\alpha}^{(i)}\left(x_{\tau(n)}^{(i-1)}\right)\right\|=0(i \in \mathcal{I}), \\
& \lim _{n \rightarrow \infty}\left\|x_{\tau(n)}-x_{\tau(n)}^{(i-1)}\right\|=0(i \in \mathcal{I}), \\
& \lim _{n \rightarrow \infty}\left\|x_{\tau(n)}-Q_{\alpha}^{(i)}\left(x_{\tau(n)}^{(i-1)}\right)\right\|=0(i \in \mathcal{I}) .
\end{aligned}
$$

Inequality (4.10) and $\lambda_{\tau(n)}>0\left(n \geq m_{1}\right)$ mean that $N_{\tau(n)}\left(x_{0}^{*}\right)<0\left(n \geq m_{1}\right)$; i.e., for all $n \geq m_{1}$,

$$
\begin{aligned}
f\left(x_{\tau(n)}\right)-f^{\star}< & \sqrt{N_{1}} \sum_{i \in \mathcal{I}}\left\|Q_{\alpha}^{(i)}\left(x_{\tau(n)}^{(i-1)}\right)-x_{\tau(n)}^{(i-1)}\right\|+I N_{1} \lambda_{\tau(n)} \\
& +N_{2} \sum_{i \in \mathcal{I}}\left\|Q_{\alpha}^{(i)}\left(x_{\tau(n)}^{(i-1)}\right)-x_{\tau(n)}\right\| .
\end{aligned}
$$

Accordingly, (C2), (4.12), and (4.14) imply that $\lim _{\sup } \sup _{n \rightarrow \infty} f\left(x_{\tau(n)}\right) \leq f^{\star}$, which implies that, for any subsequence $\left(x_{\tau\left(n_{k}\right)}\right)_{k \in \mathbb{N}}\left(\subset\left(x_{\tau(n)}\right)_{n \geq m_{1}}\right), \lim _{k \rightarrow \infty} f\left(x_{\tau\left(n_{k}\right)}\right) \leq \limsup _{n \rightarrow \infty} f\left(x_{\tau(n)}\right) \leq$ $f^{\star}$. From the boundedness of $\left(x_{\tau\left(n_{k}\right)}\right)_{k \in \mathbb{N}}$, there is $\left(x_{\tau\left(n_{k_{l}}\right)}\right)_{l \in \mathbb{N}}\left(\subset\left(x_{\tau\left(n_{k}\right)}\right)_{k \in \mathbb{N}}\right)$, which weakly converges to $x_{\star} \in H$. Equation (4.13) implies that $\left(x_{\tau\left(n_{k_{l}}\right)}^{(i-1)}\right)_{l \in \mathbb{N}}(i \in \mathcal{I})$ weakly converges to $x_{\star}$. Hence, (A6) and (4.11) lead to $x_{\star} \in X$. The same discussion as in the proof of Theorem 3.2 guarantees that $x_{\star} \in X^{\star}$. Therefore, there exists a subsequence of $\left(x_{n}^{(i)}\right)_{n \in \mathbb{N}}(i \in \mathcal{I})$ that weakly converges to a point in $X^{\star}$.

Let us assume that either (i) or (ii) is satisfied. A discussion similar to the one for proving the strong convergence of $\left(x_{n}\right)_{n \in \mathbb{N}}$ in Algorithm 3.1 to a unique point in $X^{\star}$ guarantees that $\left(x_{n}\right)_{n \in \mathbb{N}}$ in Algorithm 4.1 strongly converges to $x^{\star} \in X^{\star}$. From $\lim _{n \rightarrow \infty}\left\|x_{n}-x_{n}^{(i-1)}\right\|=0$ $(i \in \mathcal{I})$ (see (4.7) and (4.13)), we can conclude that $\left(x_{n}^{(i)}\right)(i \in \mathcal{I})$ strongly converges to $x^{\star}$. This completes the proof. 
Regarding the relationship between the proposed algorithms (Algorithms 3.1] and 4.1) and the distributed random projection method [20, we have the following remark.

Remark 4.1. Suppose that user $i$ 's objective function $f^{(i)}$ is convex and differentiable and that user $i$ 's constraint set $C^{(i)}$ is defined as the intersection of finitely many simple closed convex constraints; i.e.,

$$
C^{(i)}:=\bigcap_{k \in \mathcal{J}^{(i)}} C_{k}^{(i)},
$$

where $\mathcal{J}^{(i)}$ is finite and $C_{k}^{(i)}\left(k \in \mathcal{J}^{(i)}\right)$ is a nonempty, closed convex set of $\mathbb{R}^{N}$ such that $P_{C_{k}^{(i)}}$ can be computed efficiently. At iteration $n$ of the method [20], user $i$ calculates the weighted average of the $x_{n}^{(j)}$ received from its local neighbors $j$ and determines the iteration value by using the gradient information of its own objective function and the metric projection onto a constraint $C_{\Omega_{n}^{(i)}}^{(i)}\left(\Omega_{n}^{(i)} \in \mathcal{J}^{(i)}\right)$ selected randomly from its constraint set $C^{(i)}$; i.e.,

$$
\begin{aligned}
v_{n}^{(i)} & :=\sum_{j \in N_{n}^{(i)}} w_{i j, n} x_{n}^{(j)}, \\
x_{n+1}^{(i)} & :=P_{C_{\Omega_{n}^{(i)}}^{(i)}}\left(v_{n}^{(i)}-\alpha_{n} \nabla f^{(i)}\left(v_{n}^{(i)}\right)\right),
\end{aligned}
$$

where $N_{n}^{(i)}$ stands for the set of user $i$ and the users that send information to user $i, w_{i j, n} \geq 0$ $\left(j \in N_{n}^{(i)}\right)$ with $\sum_{j \in N_{n}^{(i)}} w_{i j, n}=1(i \in \mathcal{I})$, and $\alpha_{n}>0$. Proposition 1 in [20] indicates that, under certain assumptions, the sequence $\left(x_{n}^{(i)}\right)_{n \in \mathbb{N}}(i \in \mathcal{I})$ generated by Algorithm (4.16) converges almost surely to the minimizer of $\sum_{i \in \mathcal{I}} f^{(i)}$ over $\bigcap_{i \in \mathcal{I}} C^{(i)}$.

Algorithm 3.1 (resp. Algorithm 4.1) can be applied to the problem considered in [20. under Assumption (A5) (resp. Assumption (A7)) and the assumption that user $i$ can use all $P_{C_{k}^{(i)}}\left(k \in \mathcal{J}^{(i)}\right)$ at each iteration. Since the product of metric projections or the weighted average of metric projections is a special case of a quasi-nonexpansive mapping, $Q^{(i)}$ in Algorithms 3.1 and 4.1 can be given, for example, by

$$
Q^{(i)}:=\prod_{k \in \mathcal{J}^{(i)}} P_{C_{k}^{(i)}} \text { or } Q^{(i)}:=\sum_{k \in \mathcal{J}^{(i)}} w_{k}^{(i)} P_{C_{k}^{(i)}}
$$

where $\left(w_{k}^{(i)}\right)_{k \in \mathcal{J}^{(i)}}(i \in \mathcal{I})$ satisfies $\sum_{k \in \mathcal{J}^{(i)}} w_{k}^{(i)}=1$.

\subsection{Convergence rate analysis of Algorithm 4.1 with di- minishing step size}

Here we first discuss the rate of convergence of Algorithm 4.1 for unconstrained nonsmooth convex optimization.

Corollary 4.1. Consider Problem 2.1] when $Q^{(i)}=\operatorname{Id}(i \in \mathcal{I})$ and suppose that the assumptions in Theorem 4.2 hold. Then, for a large enough $n \in \mathbb{N}$,

$$
f\left(x_{n}\right)-f^{\star} \leq I\left\{\frac{(I-1)}{2} \sqrt{N_{1}} N_{2}+N_{1}\right\} \lambda_{n},
$$

where $N_{1}:=\max _{i \in \mathcal{I}} N_{1}^{(i)^{2}}<\infty, N_{2}:=\max _{i \in \mathcal{I}} N_{2}^{(i)}<\infty$, and $N_{1}^{(i)}$ and $N_{2}^{(i)}(i \in \mathcal{I})$ are defined as in Assumption 4.1 . 
Corollary 4.1 indicates that, when the same step size sequence is used, the efficiency of Algorithm 4.1 with $Q^{(i)}=\operatorname{Id}(i \in \mathcal{I})$ may decrease as the number of users $I$ increases. This can also be seen in Corollary 3.1 indicating the rate of convergence of Algorithm 3.1] with $Q^{(i)}=\operatorname{Id}(i \in \mathcal{I})$.

Proof. The triangle inequality ensures that $\left\|x_{n}-x_{n}^{(i-1)}\right\| \leq \sum_{j=1}^{i-1}\left\|x_{n}^{(j-1)}-x_{n}^{(j)}\right\|(n \in$ $\mathbb{N})$, which, together with the definition of $x_{n}^{(i)}(i \in \mathcal{I}, n \in \mathbb{N})$, implies $\left\|x_{n}-x_{n}^{(i-1)}\right\| \leq$ $\sum_{j=1}^{i-1} \sqrt{N_{1}} \lambda_{n}=(i-1) \sqrt{N_{1}} \lambda_{n}(i \in \mathcal{I}, n \in \mathbb{N})$. Accordingly, for all $n \in \mathbb{N}$,

$$
\sum_{i \in \mathcal{I}}\left\|x_{n}-x_{n}^{(i-1)}\right\| \leq \sum_{i \in \mathcal{I}}(i-1) \sqrt{N_{1}} \lambda_{n}=\frac{I(I-1)}{2} \sqrt{N_{1}} \lambda_{n}
$$

In Case 1 in the proof of Theorem 4.2, $\liminf _{n \rightarrow \infty} N_{n}\left(x^{\star}\right) \leq 0$ holds, where $N_{n}(x)$ $(n \in \mathbb{N}, x \in H)$ is defined by (4.9) and $\left\{x^{\star}\right\}=X^{\star}$. The same discussion as in the proof of Corollary 3.1 leads to the existence of $k_{1} \in \mathbb{N}$ such that, for all $n \geq k_{1}, N_{n}\left(x^{\star}\right) \leq 0$. From $Q^{(i)}=\operatorname{Id}(i \in \mathcal{I})$, for all $n \geq k_{1}, N_{n}\left(x^{\star}\right)=f\left(x_{n}\right)-f^{\star}-N_{2} \sum_{i \in \mathcal{I}}\left\|x_{n}-x_{n}^{(i-1)}\right\|-I N_{1} \lambda_{n} \leq 0$. Hence, (4.17) implies that, for all $n \geq k_{1}$,

$$
\begin{aligned}
f\left(x_{n}\right)-f^{\star} & \leq N_{2} \sum_{i \in \mathcal{I}}\left\|x_{n}-x_{n}^{(i-1)}\right\|+I N_{1} \lambda_{n} \\
& \leq \frac{I(I-1)}{2} \sqrt{N_{1}} N_{2} \lambda_{n}+I N_{1} \lambda_{n} .
\end{aligned}
$$

In Case 2 in the proof of Theorem 4.2 the condition $Q^{(i)}=\operatorname{Id}(i \in \mathcal{I})$, (4.15), and (4.17) lead to the existence of $k_{2} \in \mathbb{N}$ such that, for all $n \geq k_{2}, f\left(x_{\tau(n)}\right)-f^{\star}<N_{2} \sum_{i \in \mathcal{I}} \| x_{\tau(n)}-$ $x_{\tau(n)}^{(i-1)} \|+I N_{1} \lambda_{\tau(n)} \leq(I(I-1) / 2) \sqrt{N_{1}} N_{2} \lambda_{\tau(n)}+I N_{1} \lambda_{\tau(n)}$. This completes the proof.

The following corollary establishes the rate of convergence of Algorithm 4.1 for constrained nonsmooth convex optimization under specific conditions.

Corollary 4.2. Suppose that the assumptions in Theorem 4.2 hold. If there exists $\beta^{(i)}>0$ $(i \in \mathcal{I})$ such that $\alpha^{(i)}>\beta^{(i)^{2}} /\left(\beta^{(i)^{2}}+2\right)$ and $\mathrm{d}\left(x_{n}^{(i-1)}, X\right):=\left\|x_{n}^{(i-1)}-P_{X}\left(x_{n}^{(i-1)}\right)\right\| \leq$ $\beta^{(i)}\left\|x_{n}^{(i-1)}-Q_{\alpha}^{(i)}\left(x_{n}^{(i-1)}\right)\right\|(i \in \mathcal{I}, n \in \mathbb{N})$ and if $\left(\left\|x_{n}^{(i-1)}-Q^{(i)}\left(x_{n}^{(i-1)}\right)\right\|\right)_{n \in \mathbb{N}}(i \in \mathcal{I})$ is monotone decreasing, then, for all $i \in \mathcal{I}$ and for all $n \in \mathbb{N}$,

$$
\left\|x_{n}^{(i-1)}-Q^{(i)}\left(x_{n}^{(i-1)}\right)\right\|^{2} \leq \frac{\mathrm{d}\left(x_{0}, X\right)^{2}+3 I N_{1} \sum_{k=0}^{n} \lambda_{k}^{2}}{\left(1-\alpha^{(i)}\right)\left\{\left(\beta^{(i)^{2}}+2\right) \alpha^{(i)}-\beta^{(i)^{2}}\right\}(n+1)},
$$

where $\left(\lambda_{n}\right)_{n \in \mathbb{N}}$ satisfies $\sum_{n=0}^{\infty} \lambda_{n}^{2}<\infty, N_{1}:=\max _{i \in \mathcal{I}} N_{1}^{(i)^{2}}<\infty$, and $N_{1}^{(i)}(i \in \mathcal{I})$ is defined as in Assumption [4.1. Moreover, for a large enough $n \in \mathbb{N}$,

$$
f\left(x_{n}\right)-f^{\star} \leq I\left\{\left(\sqrt{N_{1}}+\frac{(I+1) N_{2}}{2}\right) \sqrt{\frac{N_{3}}{n+1}}+\left(\frac{(I-1) \sqrt{N_{1}} N_{2}}{2}+N_{1}\right) \lambda_{n}\right\},
$$

where $N_{2}:=\max _{i \in \mathcal{I}} N_{2}^{(i)}<\infty, N_{2}^{(i)}(i \in \mathcal{I})$ is defined as in Assumption 4.1. $N_{3}:=$ $\max _{i \in \mathcal{I}} N_{3}^{(i)}<\infty$, and $N_{3}^{(i)}:=\left(\mathrm{d}\left(x_{0}, X\right)^{2}+3 I N_{1} \sum_{k=0}^{\infty} \lambda_{k}^{2}\right) /\left(\left(1-\alpha^{(i)}\right)\left\{\left(\beta^{(i)^{2}}+2\right) \alpha^{(i)}-\beta^{(i)^{2}}\right\}\right)$ $(i \in \mathcal{I})$. 
Consider the case where $\alpha^{(i)}:=1 / 2$ and $Q^{(i)}:=\left(1 /\left(1-\alpha^{(i)}\right)\right)\left(P_{X}-\alpha^{(i)} \operatorname{Id}\right)(i \in \mathcal{I})$; i.e., $Q_{\alpha}^{(i)}=P_{X}(i \in \mathcal{I})$ and $Q^{(i)}(i \in \mathcal{I})$ is nonexpansive [3, Proposition 4.25]. Then, $\beta^{(i)}=1$ can be chosen such that $\alpha^{(i)}=1 / 2>\beta^{(i)^{2}} /\left(\beta^{(i)^{2}}+2\right)=1 / 3$ and $\mathrm{d}\left(x_{n}^{(i-1)}, X\right)=$ $\beta^{(i)}\left\|x_{n}^{(i-1)}-Q_{\alpha}^{(i)}\left(x_{n}^{(i-1)}\right)\right\|(i \in \mathcal{I}, n \in \mathbb{N})$.

Corollary 4.2 implies that, when the same step size sequence is used, the efficiency of Algorithm 4.1 may decrease as the number of users $I$ increases. This can also be seen in Corollary 3.2. indicating the rate of convergence of Algorithm 3.1 for constrained nonsmooth convex optimization.

Proof. Define $\mathrm{d}(x, X):=\left\|x-P_{X}(x)\right\|(x \in H)$ and $z_{n}^{(i)}:=P_{X}\left(x_{n}^{(i)}\right)(i \in \mathcal{I}, n \in \mathbb{N})$. From (4.1), for all $i \in \mathcal{I}$ and for all $n \in \mathbb{N}$,

$$
\begin{aligned}
\left\|x_{n}^{(i)}-z_{n}^{(i-1)}\right\|^{2} \leq & \mathrm{d}\left(x_{n}^{(i-1)}, X\right)^{2}-2 \alpha^{(i)}\left(1-\alpha^{(i)}\right)\left\|x_{n}^{(i-1)}-Q^{(i)}\left(x_{n}^{(i-1)}\right)\right\|^{2} \\
& +2 N_{1} \lambda_{n}^{2}+2 \lambda_{n}\left\langle z_{n}^{(i-1)}-x_{n}^{(i-1)}, g_{n}^{(i)}\right\rangle
\end{aligned}
$$

which, together with $\mathrm{d}\left(x_{n}^{(i)}, X\right) \leq\left\|x_{n}^{(i)}-z_{n}^{(i-1)}\right\|(i \in \mathcal{I}, n \in \mathbb{N})$ and the definition of $x_{n}$ $(n \in \mathbb{N})$, implies that, for all $n \in \mathbb{N}$,

$$
\begin{aligned}
\mathrm{d}\left(x_{n+1}, X\right)^{2} \leq & \mathrm{d}\left(x_{n}, X\right)^{2}-2 \sum_{i \in \mathcal{I}} \alpha^{(i)}\left(1-\alpha^{(i)}\right)\left\|x_{n}^{(i-1)}-Q^{(i)}\left(x_{n}^{(i-1)}\right)\right\|^{2} \\
& +2 I N_{1} \lambda_{n}^{2}+2 \lambda_{n} \sum_{i \in \mathcal{I}}\left\langle z_{n}^{(i-1)}-x_{n}^{(i-1)}, g_{n}^{(i)}\right\rangle .
\end{aligned}
$$

Furthermore, the Cauchy-Schwarz inequality and $2\|x\|\|y\| \leq\|x\|^{2}+\|y\|^{2}(x, y \in H)$ ensure that, for all $i \in \mathcal{I}$ and for all $n \in \mathbb{N}, 2\left\langle z_{n}^{(i-1)}-x_{n}^{(i-1)}, \lambda_{n} g_{n}^{(i)}\right\rangle \leq\left\|z_{n}^{(i-1)}-x_{n}^{(i-1)}\right\|^{2}+\lambda_{n}^{2}\left\|g_{n}^{(i)}\right\|^{2}$, which, together with the definition of $N_{1}$ and $\left\|x_{n}^{(i-1)}-z_{n}^{(i-1)}\right\| \leq \beta^{(i)}\left\|x_{n}^{(i-1)}-Q_{\alpha}^{(i)}\left(x_{n}^{(i-1)}\right)\right\|$ $(i \in \mathcal{I}, n \in \mathbb{N})$, implies that

$$
\begin{aligned}
2 \sum_{n=0}^{N} \sum_{i \in \mathcal{I}}\left\langle z_{n}^{(i-1)}-x_{n}^{(i-1)}, \lambda_{n} g_{n}^{(i)}\right\rangle \leq & \sum_{n=0}^{N} \sum_{i \in \mathcal{I}} \beta^{(i)^{2}}\left\|x_{n}^{(i-1)}-Q_{\alpha}^{(i)}\left(x_{n}^{(i-1)}\right)\right\|^{2} \\
& +I N_{1} \sum_{n=0}^{N} \lambda_{n}^{2} .
\end{aligned}
$$

Accordingly, for all $N \in \mathbb{N}$,

$$
\begin{aligned}
& \sum_{n=0}^{N} \sum_{i \in \mathcal{I}}\left(1-\alpha^{(i)}\right)\left\{\left(\beta^{(i)^{2}}+2\right) \alpha^{(i)}-\beta^{(i)^{2}}\right\}\left\|x_{n}^{(i-1)}-Q^{(i)}\left(x_{n}^{(i-1)}\right)\right\|^{2} \\
& \quad \leq \mathrm{d}\left(x_{0}, X\right)^{2}+3 I N_{1} \sum_{n=0}^{N} \lambda_{n}^{2} .
\end{aligned}
$$

Since $\left(\left\|x_{n}^{(i-1)}-Q^{(i)}\left(x_{n}^{(i-1)}\right)\right\|\right)_{n \in \mathbb{N}}(i \in \mathcal{I})$ is monotone decreasing, for all $j \in \mathcal{I}$ and for all 
$N \in \mathbb{N}$

$$
\begin{aligned}
& (N+1)\left(1-\alpha^{(j)}\right)\left\{\left(\beta^{(j)^{2}}+2\right) \alpha^{(j)}-\beta^{(j)^{2}}\right\}\left\|x_{N}^{(j-1)}-Q^{(j)}\left(x_{N}^{(j-1)}\right)\right\|^{2} \\
\leq & (N+1) \sum_{i \in \mathcal{I}}\left(1-\alpha^{(i)}\right)\left\{\left(\beta^{(i)^{2}}+2\right) \alpha^{(i)}-\beta^{(i)^{2}}\right\}\left\|x_{N}^{(i-1)}-Q^{(i)}\left(x_{N}^{(i-1)}\right)\right\|^{2} \\
\leq & \mathrm{d}\left(x_{0}, X\right)^{2}+3 I N_{1} \sum_{n=0}^{N} \lambda_{n}^{2},
\end{aligned}
$$

which implies that, for all $j \in \mathcal{I}$ and for all $N \in \mathbb{N}$,

$$
\left\|x_{N}^{(j-1)}-Q^{(j)}\left(x_{N}^{(j-1)}\right)\right\|^{2} \leq \frac{\mathrm{d}\left(x_{0}, X\right)^{2}+3 I N_{1} \sum_{n=0}^{N} \lambda_{n}^{2}}{\left(1-\alpha^{(j)}\right)\left\{\left(\beta^{(j)^{2}}+2\right) \alpha^{(j)}-\beta^{(j)^{2}}\right\}(N+1)} .
$$

Since $\left\|x_{n}^{(i-1)}-x_{n}^{(i)}\right\| \leq\left\|x_{n}^{(i-1)}-Q_{\alpha}^{(i)}\left(x_{n}^{(i-1)}\right)\right\|+\sqrt{N_{1}} \lambda_{n}(i \in \mathcal{I}, n \in \mathbb{N})$, for all $i \in \mathcal{I}$ and $n \in \mathbb{N}$,

$$
\left\|x_{n}^{(i-1)}-x_{n}^{(i)}\right\| \leq\left(1-\alpha^{(i)}\right) \sqrt{\frac{N_{3}}{n+1}}+\sqrt{N_{1}} \lambda_{n} .
$$

Moreover, since the triangle inequality implies that $\left\|x_{n}-x_{n}^{(i-1)}\right\| \leq \sum_{j=1}^{i-1}\left\|x_{n}^{(j-1)}-x_{n}^{(j)}\right\|$ $(i \in \mathcal{I}, n \in \mathbb{N})$, for all $n \in \mathbb{N}$,

$$
\begin{aligned}
\sum_{i \in \mathcal{I}}\left\|x_{n}-x_{n}^{(i-1)}\right\| & \leq \sum_{i \in \mathcal{I}} \sum_{j=1}^{i-1}\left\{\left(1-\alpha^{(j)}\right) \sqrt{\frac{N_{3}}{n+1}}+\sqrt{N_{1}} \lambda_{n}\right\} \\
& \leq \frac{I(I-1)}{2}\left(\sqrt{\frac{N_{3}}{n+1}}+\sqrt{N_{1}} \lambda_{n}\right) .
\end{aligned}
$$

Accordingly, for all $n \in \mathbb{N}$,

$$
\begin{aligned}
\sum_{i \in \mathcal{I}}\left\|x_{n}-Q_{\alpha}^{(i)}\left(x_{n}^{(i-1)}\right)\right\| & \leq \sum_{i \in \mathcal{I}}\left\|x_{n}^{(i-1)}-Q_{\alpha}^{(i)}\left(x_{n}^{(i-1)}\right)\right\|+\sum_{i \in \mathcal{I}}\left\|x_{n}-x_{n}^{(i-1)}\right\| \\
& \leq \frac{I}{2}\left\{(I+1) \sqrt{\frac{N_{3}}{n+1}}+(I-1) \sqrt{N_{1}} \lambda_{n}\right\} .
\end{aligned}
$$

In Case 1 in the proof of Theorem 4.2. $\liminf _{n \rightarrow \infty} N_{n}\left(x^{\star}\right) \leq 0$, where $\left\{x^{\star}\right\}=X^{\star}$. A discussion similar to the one for proving $M_{n}\left(x^{\star}\right) \leq 0\left(n \geq k_{1}\right)$ (see proof of Corollary 3.1) guarantees that there exists $k_{3} \in \mathbb{N}$ such that, for all $n \geq k_{3}, N_{n}\left(x^{\star}\right)=f\left(x_{n}\right)$ $f^{\star}-\sqrt{N_{1}} \sum_{i \in \mathcal{I}}\left\|x_{n}^{(i-1)}-Q_{\alpha}^{(i)}\left(x_{n}^{(i-1)}\right)\right\|-N_{2} \sum_{i \in \mathcal{I}}\left\|Q_{\alpha}^{(i)}\left(x_{n}^{(i-1)}\right)-x_{n}\right\|-I N_{1} \lambda_{n} \leq 0$. From (4.15) in Case 2 in the proof of Theorem 4.2 there exists $k_{4} \in \mathbb{N}$ such that, for all $n \geq k_{4}$, $f\left(x_{\tau(n)}\right)-f^{\star}<\sqrt{N_{1}} \sum_{i \in \mathcal{I}}\left\|x_{\tau(n)}^{(i-1)}-Q_{\alpha}^{(i)}\left(x_{\tau(n)}^{(i-1)}\right)\right\|+N_{2} \sum_{i \in \mathcal{I}}\left\|Q_{\alpha}^{(i)}\left(x_{\tau(n)}^{(i-1)}\right)-x_{\tau(n)}\right\|+I N_{1} \lambda_{n}$. Therefore, from $\left\|x_{n}^{(i-1)}-Q_{\alpha}^{(i)}\left(x_{n}^{(i-1)}\right)\right\|^{2} \leq N_{3} /(n+1)(n \in \mathbb{N})$ and (4.19), for a large enough 
$n \in \mathbb{N}$,

$$
\begin{aligned}
& f\left(x_{n}\right)-f^{\star} \\
\leq & \sqrt{N_{1}} \sum_{i \in \mathcal{I}}\left\|x_{n}^{(i-1)}-Q_{\alpha}^{(i)}\left(x_{n}^{(i-1)}\right)\right\|+N_{2} \sum_{i \in \mathcal{I}}\left\|Q_{\alpha}^{(i)}\left(x_{n}^{(i-1)}\right)-x_{n}\right\|+I N_{1} \lambda_{n} \\
\leq & \sqrt{N_{1}} \sum_{i \in \mathcal{I}}\left(1-\alpha^{(i)}\right) \sqrt{\frac{N_{3}}{n+1}}+I N_{1} \lambda_{n} \\
& +N_{2} \frac{I}{2}\left\{(I+1) \sqrt{\frac{N_{3}}{n+1}}+(I-1) \sqrt{N_{1}} \lambda_{n}\right\} \\
\leq & I\left\{\left(\sqrt{N_{1}}+\frac{(I+1) N_{2}}{2}\right) \sqrt{\frac{N_{3}}{n+1}}+\left(\frac{(I-1) \sqrt{N_{1}} N_{2}}{2}+N_{1}\right) \lambda_{n}\right\} .
\end{aligned}
$$

This completes the proof.

\section{$5 \quad$ Numerical Examples}

This section considers the following problem over the intersection of sublevel sets of convex functions [13, Section 3.2] and numerically compares Algorithms 3.1 and 4.1 with the method in [13, (2.1), (3.1), (3.14), (4.3)].

Problem 5.1. Let $f^{(i)}: \mathbb{R}^{N} \rightarrow \mathbb{R}$ and $g^{(i)}: \mathbb{R}^{N} \rightarrow \mathbb{R}(i \in \mathcal{I})$ be convex.

$$
\text { Minimize } f(x):=\sum_{i \in \mathcal{I}} f^{(i)}(x) \text { subject to } x \in X:=\bigcap_{i \in \mathcal{I}} \operatorname{lev} \leq 0 g^{(i)} \neq \emptyset \text {, }
$$

where $\operatorname{lev}_{\leq 0} g^{(i)}:=\left\{x \in \mathbb{R}^{N}: g^{(i)}(x) \leq 0\right\}$.

Let us define the subgradient projection [2, Proposition 2.3], [34, Subchapter 4.3] relative to $g^{(i)}(i \in \mathcal{I})$ for all $x \in \mathbb{R}^{N}$ by

$$
Q_{\mathrm{sp}}^{(i)}(x):= \begin{cases}x-\frac{g^{(i)}(x)}{\left\|z^{(i)}(x)\right\|^{2}} z^{(i)}(x) & \text { if } g^{(i)}(x)>0, \\ x & \text { otherwise, }\end{cases}
$$

where $z^{(i)}(x) \in \partial g^{(i)}(x)\left(i \in \mathcal{I}, x \in \mathbb{R}^{N}\right)$. The mapping $Q_{\mathrm{sp}}^{(i)}(i \in \mathcal{I})$ is quasi-firmly nonexpansive, and $\operatorname{Id}-Q_{\mathrm{sp}}^{(i)}(i \in \mathcal{I})$ is demiclosed in the sense of the Euclidean space setting [1, Lemma 3.1]. Moreover, $\operatorname{Fix}\left(Q_{\mathrm{sp}}^{(i)}\right)=\operatorname{lev}_{\leq 0} g^{(i)}$. Hence, Problem 5.1 is an example of Problem 2.1 that can be solved by Algorithms 3.1 and 4.1 (see Theorems 3.1, 3.2, 4.1, and 4.2).

Here it is assumed that $\operatorname{lev}_{\leq 0} g^{(p)}$ is bounded for some $p \in \mathcal{I}$ (see also 13, Proposition 3.4]). Accordingly, a closed ball $Y$ with a large enough radius can be chosen so that $Y \supset$ $\operatorname{lev}_{\leq 0} g^{(p)} \supset X$. Hence, setting $X^{(i)}:=Y(i \in \mathcal{I})$ in (3.1) satisfies Assumptions 3.2 and 4.2 ,

The following is the incremental subgradient method (ISM) [13, (2.1), (3.1), (3.14), 
(4.3)] used for solving Problem 5.1 given $x_{0} \in \mathbb{R}^{N}$ and $\left(\lambda_{n}\right)_{n \in \mathbb{N}}(\subset(0, \infty))$ :

$$
\left\{\begin{array}{l}
x_{n}^{(0)}:=x_{n}, \\
x_{n}^{(i)}:=P_{Y}\left(x_{n}^{(i-1)}-\lambda_{n} g_{n}^{(i)}\right), g_{n}^{(i)} \in \partial f^{(i)}\left(x_{n}^{(i-1)}\right) \quad(i \in \mathcal{I}), \\
y_{n}^{(0)}:=x_{n}^{(I)} \\
y_{n}^{(i)}:=Q_{\mathrm{sp}}^{(i)}\left(y_{n}^{(i-1)}\right) \quad(i \in \mathcal{I}), \\
x_{n+1}:=y_{n}^{(I)} .
\end{array}\right.
$$

Theorem 2.5 in [13] guarantees that, if $\left(\left\|x_{n}-P_{X}\left(x_{n}\right)\right\|\right)_{n \in \mathbb{N}}$ is bounded and if $\lim _{n \rightarrow \infty} \max \left\{0, f\left(P_{X}\left(x_{n}\right)\right)-\right.$ $\left.f\left(x_{n}\right)\right\}=0,\left(x_{n}\right)_{n \in \mathbb{N}}$ generated by (5.1) with (C2) and (C3) satisfies $\lim _{n \rightarrow \infty}\left\|x_{n}-P_{X}\left(x_{n}\right)\right\|=$ 0 and $\lim _{n \rightarrow \infty} f\left(x_{n}\right)=f^{\star}$.

In an experiment, we define that, for all $i \in \mathcal{I}, f^{(i)}(x):=\left|a^{(i)} x+b^{(i)}\right|(x \in \mathbb{R})$ and $g^{(i)}(x):=\left\langle c^{(i)}, x\right\rangle+d^{(i)}\left(\left\langle c^{(i)}, x\right\rangle>-d^{(i)}\right)$ or $0\left(\left\langle c^{(i)}, x\right\rangle \leq-d^{(i)}\right)$, where $a^{(i)}>0, b^{(i)}, d^{(i)} \in$ $\mathbb{R}$, and $c^{(i)} \in\left\{x:=\left(x_{1}, x_{2}, \ldots, x_{I}\right) \in \mathbb{R}^{I}: x_{i}>0(i \in \mathcal{I})\right\}$. We modified $g^{(1)}(x):=\|x\|-2 C$, where $C>0$, to satisfy $\operatorname{lev}_{\leq 0} g^{(1)} \subset Y:=\left\{x \in \mathbb{R}^{I}:\|x\| \leq 2 C\right\}$. The experiment was one using a 27-inch iMac with a $3.20 \mathrm{GHz}$ Intel(R) Core(TM) i5-4570 CPU processor, 24 GB, $1600 \mathrm{MHz}$ DDR3 memory, and Mac OSX Yosemite (Version 10.10.3) operating system. ISM (Algorithm (5.1)), Algorithm 3.1 and Algorithm 4.1 were written in Python 3.4.3, and gnuplot 5.0 (patchlevel 0) was used to graph the results. We set $I:=2,8,16,64,256$ and $\alpha^{(i)}:=1 / 2(i \in \mathcal{I})$ and used $a^{(i)} \in(0,100], b^{(i)} \in[-100,100], c^{(i)}$ with $\left\|c^{(i)}\right\|=1$, $d^{(i)} \in[-\sqrt[I]{C}, \sqrt[I]{C}], \bar{a}^{(i)} \in \partial f^{(i)}\left(-b^{(i)} / a^{(i)}\right)$, and $\bar{c}^{(i)} \in \partial g^{(i)}(x)\left(\left\langle c^{(i)}, x\right\rangle=-d^{(i)}\right)$ generated randomly by numpy.randon 5 (a Mersenne Twister pseudo-random number generator).

To see how the choice of step size affects the convergence rate of the algorithms, we used

$$
\begin{aligned}
& \text { Constant step sizes: } \lambda_{n}:=10^{-3}, 10^{-5}(n \in \mathbb{N}), \\
& \text { Diminishing step sizes: } \lambda_{n}:=\frac{10^{-3}}{(n+1)^{a}}(a:=1,0.1,0.01, n \in \mathbb{N}) .
\end{aligned}
$$

From Theorems 3.1 and 4.1, it can be expected that Algorithms 3.1 and 4.1 with small enough constant step sizes approximate solutions to Problem 5.1. Numerical results in 14, 17 indicate that the existing fixed point optimization algorithms with small step sizes (e.g., $\left.\lambda_{n}:=10^{-2} /(n+1)^{a}, 10^{-3} /(n+1)^{a}, 10^{-5} /(n+1)^{a}(a:=0.1,0.01, n \in \mathbb{N})\right)$ have fast convergence. Accordingly, the experiment described in this section used the step sizes in (5.2). We also found that, under the same conditions as in the above paragraph, ISM, Algorithm 3.1, and Algorithm 4.1 when $\lambda_{n}:=10^{-3} /(n+1)^{a}$ and $\lambda_{n}:=10^{-5} /(n+1)^{a}$ $(a:=0.1,0.01, n \in \mathbb{N})$ perform better than when $\lambda_{n}:=1 /(n+1)^{a}(a=0.1,0.01, n \in \mathbb{N})$. Only the results for the step sizes in (5.2) are given due to lack of space. The step size $\lambda_{n}:=10^{-3} /(n+1)(n \in \mathbb{N})$ satisfying $\sum_{n=0}^{\infty} \lambda_{n}^{2}<\infty$ was used to illustrate the proposed methods' efficiency and support the convergence rate analysis of the methods (Corollaries 3.1, 3.2, 4.1, and 4.2).

We used two performance measures for each $n \in \mathbb{N}$ :

$$
D_{n}:=\frac{1}{100} \sum_{s=1}^{100} \sum_{i \in \mathcal{I}}\left\|x_{n}(s)-Q_{\mathrm{sp}}^{(i)}\left(x_{n}(s)\right)\right\|, F_{n}:=\frac{1}{100} \sum_{s=1}^{100} \sum_{i \in \mathcal{I}} f^{(i)}\left(x_{n}^{(i)}(s)\right),
$$

where $\left(x_{n}(s)\right)_{n \in \mathbb{N}}$ defined by $x_{n}(s):=\left(x_{n}^{(i)}(s)\right)(n \in \mathbb{N}, s=1,2, \ldots, 100)$ is the sequence generated by the initial point $x(s)(s=1,2, \ldots, 100)$ and each of ISM, Algorithm 3.1. and

\footnotetext{
${ }^{5}$ http://docs.scipy.org/doc/numpy/reference/routines.random.html
} 
Algorithm 4.1, If $\left(D_{n}\right)_{n \in \mathbb{N}}$ converges to 0 , they converge to some point in $\bigcap_{i \in \mathcal{I}} \operatorname{Fix}\left(Q_{\mathrm{sp}}^{(i)}\right)=$ $X$.

First, let us consider the case where $I:=64$ and $\lambda_{n}:=10^{-3}(n \in \mathbb{N})$. Figures 1 and 2 illustrate the results for ISM, Algorithm 3.1, and Algorithm 4.1, The y-axes in Figure 1 represent the value of $D_{n}$ while the y-axes in Figure 2 represent the value of $F_{n}$. The x-axes in Figures 1(a) and 2(a) represent the number of iterations while the x-axes in Figures 1(b) and 2(b) represent elapsed time. Figure 1 shows that $\left(D_{n}\right)_{n \in \mathbb{N}}$ generated by Algorithm 3.1 was stable and monotone decreasing while those generated by ISM and Algorithm 4.1 were unstable and approximately zero during the early iterations. Figure 2 shows that ISM, Algorithm 3.1 and Algorithm 4.1 minimized $F_{n}$.

Figure 3 and 4 illustrate the results when $I:=64$ and $\lambda_{n}:=10^{-5}(n \in \mathbb{N})$. Figures 1 and 3 show that Algorithm 3.1 when $\lambda_{n}:=10^{-5}\left(D_{1000} \approx 10^{-6}\right)$ performed slightly better than when $\lambda_{n}:=10^{-3}\left(D_{1000} \approx 10^{-4}\right)$. In particular, the figures indicate that $\left(D_{n}\right)_{n \in \mathbb{N}}$ for Algorithm 4.1] when $\lambda_{n}:=10^{-5}$ was more stable than when $\lambda_{n}:=10^{-3}$ and that the behavior of ISM when $\lambda_{n}=10^{-5}$ was unstable and almost the same as when $\lambda_{n}:=10^{-3}$. Figure 4shows that $\left(F_{n}\right)_{n \in \mathbb{N}}$ for ISM and Algorithm 4.1 decreased during the early iterations compared with $\left(F_{n}\right)_{n \in \mathbb{N}}$ for Algorithm 3.1 .

Next, let us consider the case where $I:=64$ and $\lambda_{n}:=10^{-3} /(n+1)^{0.1}(n \in \mathbb{N})$. Figure [5] shows that $\left(D_{n}\right)_{n \in \mathbb{N}}$ generated by Algorithm 3.1 was stable while those generated by ISM and Algorithm 4.1 were unstable and approximately zero during the early iterations, as in the case with $\lambda_{n}:=10^{-3}$ (Figure 1). Figure 6 shows that $F_{n}$ decreased faster with ISM and Algorithm 4.1 than with Algorithm 3.1. Figures 7 and 8 illustrate the behaviors of $D_{n}$ and $F_{n}$ when $I:=64$ and $\lambda_{n}:=10^{-3} /(n+1)^{0.01}(n \in \mathbb{N})$ and show that the behaviors were almost the same as the ones when $\lambda_{n}:=10^{-3} /(n+1)^{0.1}(n \in \mathbb{N})$ (Figures 5 and 6 ).

Let us fix the step size $\lambda_{n}:=10^{-3} /(n+1)^{0.01}(n \in \mathbb{N})$ and see how the number of users affects the efficiency of Algorithms 3.1 and 4.1. The behaviors of $D_{n}$ and $F_{n}$ for Algorithm 3.1 when $I:=16,64,256$ are illustrated in Figure 9. Although $\left(D_{n}\right)_{n \in \mathbb{N}}$ and $\left(F_{n}\right)_{n \in \mathbb{N}}$ were stable, the larger the $I$, the greater the number of iterations that were required (Figure 9(a), (c)) and the longer the elapsed time (Figure 9(b), (d)). That is, the efficiency of Algorithm 3.1 decreases as the number of users increases. The behaviors of $D_{n}$ and $F_{n}$ for Algorithm 4.1 when $I:=16,64,256$ are illustrated in Figure 10. Although $\left(D_{n}\right)_{n \geq 40}$ were unstable, $D_{10} \approx 10^{-5}$ held for the three cases (Figure $\left.10(\mathrm{a}),(\mathrm{b})\right)$, and $\left(F_{n}\right)_{n \in \mathbb{N}}$ for the three cases converged in the early stages (Figure 10(c), (d)).

Finally, let us consider the case when $\lambda_{n}:=10^{-3} /(n+1)(n \in \mathbb{N})$ and $f^{(1)}$ replaced by $f^{(1)}(x):=a^{(1)}\left\|x+b^{(1)}\right\|^{2}\left(x \in \mathbb{R}^{I}\right)$, where $a^{(1)} \in(0,100]$ and $b^{(1)} \in[-100,100]^{I}$ were chosen randomly, to support the convergence analysis of Algorithms 3.1 and 4.1 discussed in Subsections 3.2, 3.3, 4.2, and 4.3 (see also assumption (i) in Theorems 3.2 and 4.2 and condition $\sum_{n=0}^{\infty} \lambda_{n}^{2}<\infty$ in Corollaries 3.2 and 4.2). Since $f^{(1)}$ is strongly convex, Theorems 3.2 and 4.2 guarantee that Algorithms 3.1 and 4.1 converge to the solution to Problem 5.1. Moreover, Corollaries 3.2 and 4.2 indicate that, under certain assumptions, Algorithm 3.1 satisfies inequality

$$
\begin{aligned}
& \sum_{i \in \mathcal{I}}\left\|x_{n}-Q_{\mathrm{sp}}^{(i)}\left(x_{n}\right)\right\| \leq \frac{I \sqrt{I M_{3}}}{\sqrt{n+1}} \\
& f\left(x_{n}\right)-f^{\star} \leq \frac{I\left\{\left(\sqrt{M_{1}}+M_{2}\right) \sqrt{I M_{3}}+M_{1}\right\}}{\sqrt{n+1}},
\end{aligned}
$$


while Algorithm 4.1 satisfies inequality

$$
\begin{aligned}
& \sum_{i \in \mathcal{I}}\left\|x_{n}^{(i-1)}-Q_{\mathrm{sp}}^{(i)}\left(x_{n}^{(i-1)}\right)\right\| \leq \frac{I \sqrt{N_{3}}}{\sqrt{n+1}}, \\
& f\left(x_{n}\right)-f^{\star} \leq \frac{I\left\{\left(2 \sqrt{N_{1}}+(I+1) N_{2}\right) \sqrt{N_{3}}+\left((I-1) \sqrt{N_{1}} N_{2}+2 N_{1}\right)\right\}}{2 \sqrt{n+1}} .
\end{aligned}
$$

Inequalities (5.3) and (5.4) imply that the efficiencies of Algorithms 3.1 and 4.1 may decrease as the number of users $I$ increases. Figure 11 shows that $\left(D_{n}\right)_{n \in \mathbb{N}}$ generated by Algorithm 3.1 was monotone decreasing and that, the larger the $I$, the greater the number of iterations that were required (Figure11(a), (c)) and the longer the elapsed time (Figure11(b), (d)), as seen in Figure 9, This can be seen in (5.3). Figure12 illustrates the behaviors of $D_{n}$ and $F_{n}$ for Algorithm 4.1. It shows that the behaviors of Algorithm 4.1 when one $f^{(i)}$ was strongly convex were more stable than when all $f^{(i)}$ were convex (Figures 50 and 100). The strong convexity condition of $f^{(1)}$ (i.e., the uniqueness of the solution to Problem [5.1) apparently affects the stability of Algorithm 4.1. This is consistent with Theorem 4.2 and indicates that the whole sequence $\left(x_{n}\right)_{n \in \mathbb{N}}$ in Algorithm 4.1 converges when one $f^{(i)}$ is strongly convex while a subsequence of $\left(x_{n}\right)_{n \in \mathbb{N}}$ converges when all $f^{(i)}$ are convex. Although (5.4) and Figure 12 show that the efficiency of Algorithm 4.1 decreases as $I$ increases, Algorithm 4.1 has fast convergence regardless of the number of users. Furthermore, as shown by Figures 11 and 12. when $I:=2$, Algorithm 3.1 performed better than Algorithm 4.1 in the early stages. This means that Algorithm 3.1 is well suited for use when the number of users is small.

From the above discussion, we conclude that Algorithm 3.1 is robust in terms of stability regardless of the number of users and is well suited for small-scale convex optimization problems over fixed point sets of quasi-nonexpansive mappings. We also conclude that Algorithm 4.1 has fast convergence regardless of the number of users and is well suited for solving large-scale convex optimization problems over fixed point sets of quasi-nonexpansive mappings.

\section{Conclusion and Future Work}

This paper described parallel and incremental subgradient methods for minimizing the sum of nondifferentiable, convex functions over the intersection of fixed point sets of quasinonexpansive mappings in a real Hilbert space. Investigation of the convergence properties for a constant step-size rule and a diminishing step-size rule showed that, with a small constant step size, the two methods give an approximate solution to the minimization problem and that, with a diminishing sequence, the sequence generated by each of the two methods strongly converges to the solution to the minimization problem under certain assumptions. The convergence rate of the two methods was analyzed under certain situations.

This paper also numerically compared the proposed methods with an existing method for nonsmooth convex optimization over sublevel sets of convex functions. Numerical examples demonstrated that, for concrete convex optimization problems when the number of users is fixed, the parallel subgradient method with a constant or diminishing step size is more stable than the incremental subgradient method with the same step size while the incremental subgradient method has faster convergence. The numerical examples also demonstrated that the efficiency of the parallel subgradient method decreased as the number of users increased while the incremental subgradient method was robust even with a large number of users. 
The proposed methods work well only when each user makes the best use of its own private information while the distributed random projection method 20] works well even when each user randomly sets one projection selected from many projections. This means that consideration should be given to developing distributed random fixed point algorithms that work when one user randomly chooses one quasi-nonexpansive mapping at a time. Consideration should also be given to devising nonsmooth convex optimization algorithms that combine stability and fast convergence, in contrast to the proposed methods. For example, an algorithm combining the parallel and incremental subgradient methods could be devised on the basis of the ideas in [16]. Such an algorithm should be numerically evaluated to see whether it performs better than the two proposed methods.

Acknowledgements I am sincerely grateful to the editor, Alexander Shapiro, the anonymous associate editor, and the two anonymous reviewers for helping me improve the original manuscript. I also thank Kazuhiro Hishinuma for his input on the numerical examples.

\section{References}

[1] Bauschke, H.H., Chen, J.: A projection method for approximating fixed points of quasi nonexpansive mappings without the usual demiclosedness condition. Journal of Nonlinear and Convex Analysis 15, 129-135 (2014)

[2] Bauschke, H.H., Combettes, P.L.: A weak-to-strong convergence principle for Fejérmonotone methods in Hilbert space. Mathematics of Operations Research 26, 248-264 (2001)

[3] Bauschke, H.H., Combettes, P.L.: Convex Analysis and Monotone Operator Theory in Hilbert Spaces. Springer (2011)

[4] Bertsekas, D.P., Nedić, A., Ozdaglar, A.E.: Convex Analysis and Optimization. Athena Scientific (2003)

[5] Blatt, D., Hero, A.O., Gauchman, H.: A convergent incremental gradient method with a constant step size. SIAM Journal on Optimization 18, 29-51 (2007)

[6] Combettes, P.L.: A block-iterative surrogate constraint splitting method for quadratic signal recovery. IEEE Transactions on Signal Processing 51(7), 1771-1782 (2003)

[7] Combettes, P.L.: Iterative construction of the resolvent of a sum of maximal monotone operators. Journal of Convex Analysis 16, 727-748 (2009)

[8] Combettes, P.L., Pesquet, J.C.: A Douglas-Rachford splitting approach to nonsmooth convex variational signal recovery. IEEE Journal of Selected Topics in Signal Processing 1, 564-574 (2007)

[9] Combettes, P.L., Pesquet, J.C.: A proximal decomposition method for solving convex variational inverse problems. Inverse Problems 24, article ID 065,014 (2008)

[10] Combettes, P.L., Pesquet, J.C.: Proximal splitting methods in signal processing. In: H.H. Bauschke, R.S. Burachik, P.L. Combettes, V. Elser, D.R. Luke, H. Wolkowicz (eds.) Fixed-Point Algorithms for Inverse Problems in Science and Engineering, pp. 185-212. Springer (2011) 
[11] Eckstein, J., Bertsekas, D.P.: On the Douglas-Rachfold splitting method and proximal point algorithm for maximal monotone operators. Mathematical Programming 55, 293-318 (1992)

[12] Goebel, K., Kirk, W.A.: Topics in Metric Fixed Point Theory. Cambridge Studies in Advanced Mathematics. Cambridge University Press (1990)

[13] Helou Neto, E.S., De Pierro, A.R.: Incremental subgradients for constrained convex optimization: A unified framework and new methods. SIAM Journal on Optimization 20, 1547-1572 (2009)

[14] Iiduka, H.: Fixed point optimization algorithms for distributed optimization in networked systems. SIAM Journal on Optimization 23, 1-26 (2013)

[15] Iiduka, H.: Acceleration method for convex optimization over the fixed point set of a nonexpansive mapping. Mathematical Programming 149, 131-165 (2015)

[16] Iiduka, H., Hishinuma, K.: Acceleration method combining broadcast and incremental distributed optimization algorithms. SIAM Journal on Optimization 24, 1840-1863 (2014)

[17] Iiduka, H., Yamada, I.: A use of conjugate gradient direction for the convex optimization problem over the fixed point set of a nonexpansive mapping. SIAM Journal on Optimization 19(4), 1881-1893 (2009)

[18] Johansson, B., Rabi, M., Johansson, M.: A randomized incremental subgradient method for distributed optimization in networked systems. SIAM Journal on Optimization 20, 1157-1170 (2009)

[19] Kiwiel, K.C.: Convergence of approximate and incremental subgradient methods for convex optimization. SIAM Journal on Optimization 14, 807-840 (2004)

[20] Lee, S., Nedić, A.: Distributed random projection algorithm for convex optimization. IEEE Journal of Selected Topics in Signal Processing 7(2), 221-229 (2013)

[21] Lions, P.L., Mercier, B.: Splitting algorithms for the sum of two nonlinear operators. SIAM Journal on Numerical Analysis 16, 964-979 (1979)

[22] Lobel, I., Ozdaglar, A., Feijer, D.: Distributed multi-agent optimization with statedependent communication. Mathematical Programming 129, 255-284 (2011)

[23] Maingé, P.E.: The viscosity approximation process for quasi-nonexpansive mappings in Hilbert spaces. Computers and Mathematics with Applications 59, 74-79 (2010)

[24] Nedić, A.: Random algorithms for convex minimization problems. Mathematical Programming 129, 225-253 (2011)

[25] Nedić, A., Bertsekas, D.P.: Incremental sugradient methods for nondifferentiable optimization. SIAM Journal on Optimization 12, 109-138 (2001)

[26] Nedić, A., Olshevsky, A., Ozdaglar, A., Tsitsiklis, J.N.: On distributed averaging algorithms and quantization effects. IEEE Transactions on Automatic Control 54, 25062517 (2009)

[27] Nedić, A., Ozdaglar, A.: Distributed subgradient methods for multi agent optimization. IEEE Transactions on Automatic Control 54, 48-61 (2009) 
[28] Nedić, A., Ozdaglar, A.: Cooperative distributed multi-agent optimization. Convex Optimization in Signal Processing and Communications pp. 340-386 (2010)

[29] Opial, Z.: Weak convergence of the sequence of successive approximation for nonexpansive mappings. Bulletin of the American Mathematical Society 73, 591-597 (1967)

[30] Pesquet, J.C., Pustelnik, N.: A parallel inertial proximal optimization method. Pacific Journal of Optimization 8, 273-306 (2012)

[31] Pesquet, J.C., Repetti, A.: A class of randomized primal-dual algorithms for distributed optimization. Journal of Nonlinear and Convex Analysis (to appear)

[32] Rockafellar, R.T.: Convex Analysis. Princeton University Press (1970)

[33] Solodov, M.V., Zavriev, S.K.: Error stability properties of generalized gradient-type algorithms. Journal of Optimization Theory and Applications 98, 663-680 (1998)

[34] Vasin, V.V., Ageev, A.L.: Ill-posed problems with a priori information. V.S.P. Intl Science, Utrecht (1995)

[35] Wang, M., Bertsekas, D.P.: Incremental constraint projection-proximal methods for nonsmooth convex optimization. SIAM Journal on Optimization (to appear)

[36] Yamada, I.: The hybrid steepest descent method for the variational inequality problem over the intersection of fixed point sets of nonexpansive mappings. In: D. Butnariu, Y. Censor, S. Reich (eds.) Inherently Parallel Algorithms for Feasibility and Optimization and Their Applications, pp. 473-504. Elsevier (2001)

[37] Yamada, I., Yukawa, M., Yamagishi, M.: Minimizing the Moreau envelope of nonsmooth convex functions over the fixed point set of certain quasi-nonexpansive mappings. In: H.H. Bauschke, R.S. Burachik, P.L. Combettes, V. Elser, D.R. Luke, H. Wolkowicz (eds.) Fixed-Point Algorithms for Inverse Problems in Science and Engineering, pp. 345-390. Springer (2011)

[38] Zeidler, E.: Nonlinear Functional Analysis ans Its Applications II/B. Nonlinear Monotone Operators. Springer (1985)

[39] Zenios, S., Censor, Y.: Parallel Optimization: Theory, Algorithms, and Applications. Oxford Univ Pr on Demand (1998) 


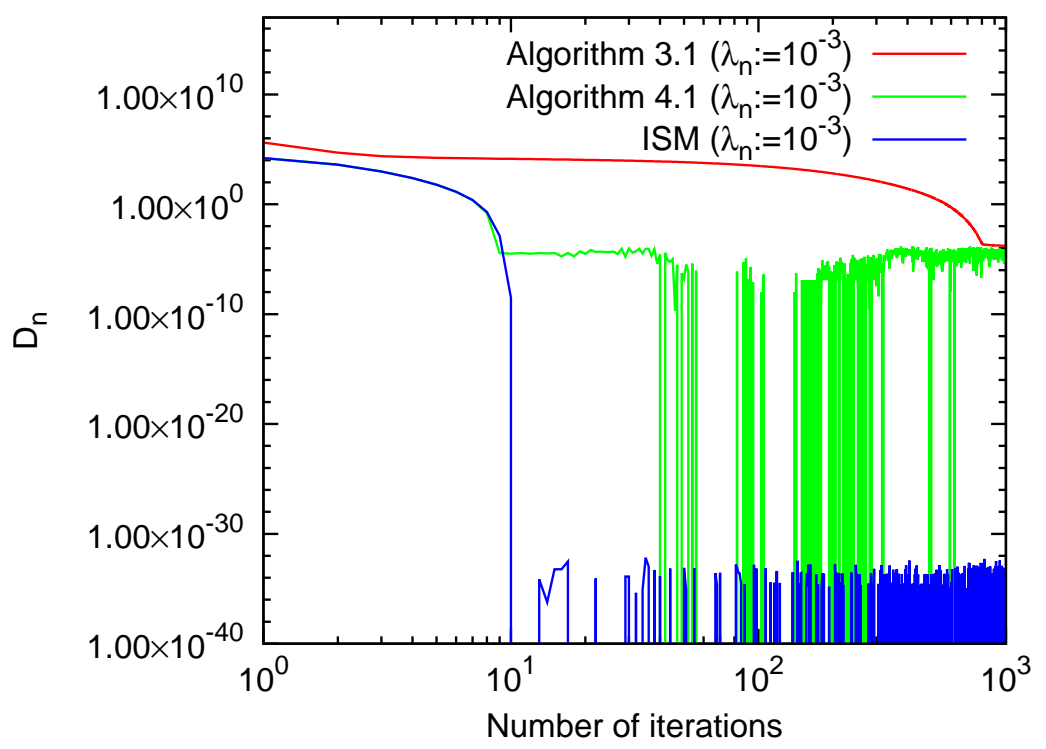

(a) $D_{n}$ vs. no. of iterations

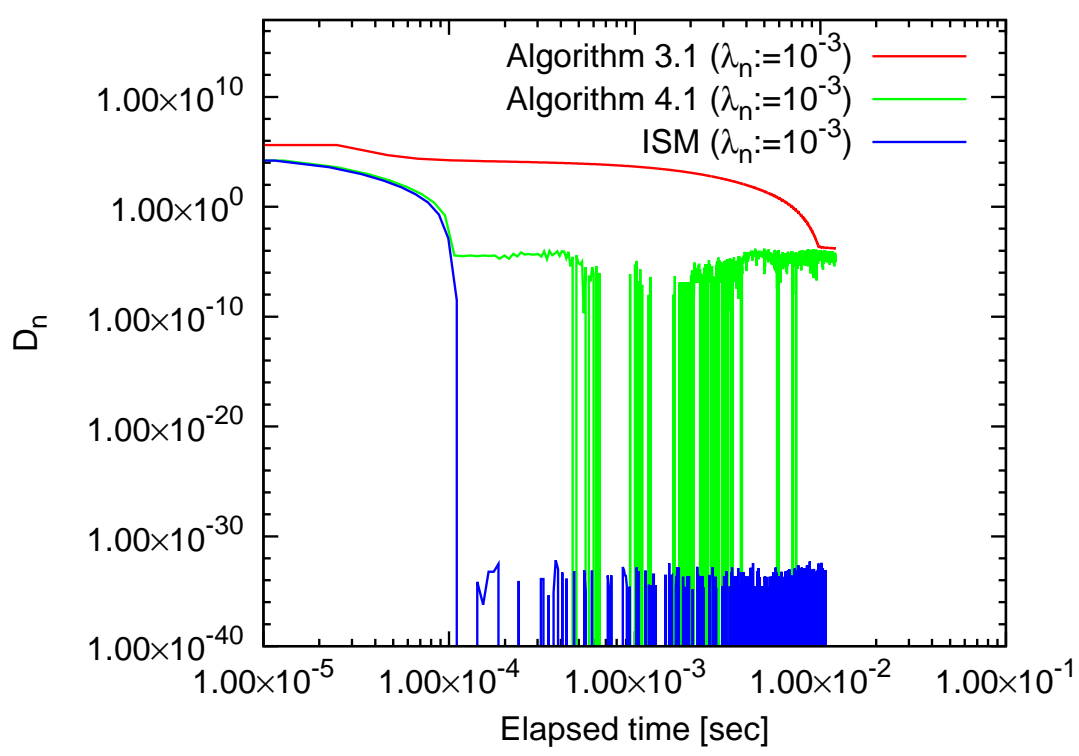

(b) $D_{n}$ vs. elapsed time

Figure 1: Behavior of $D_{n}$ for ISM, Algorithm 3.1, and Algorithm 4.1 when $\lambda_{n}:=10^{-3}$ and $I:=64$ 


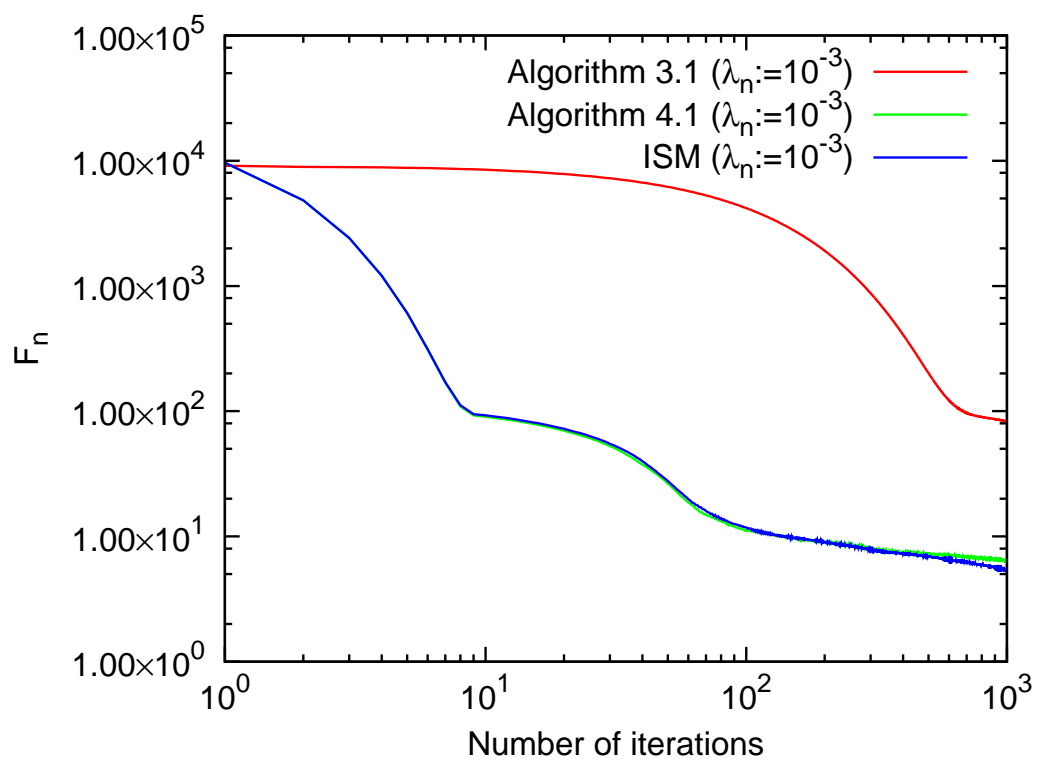

(a) $F_{n}$ vs. no. of iterations

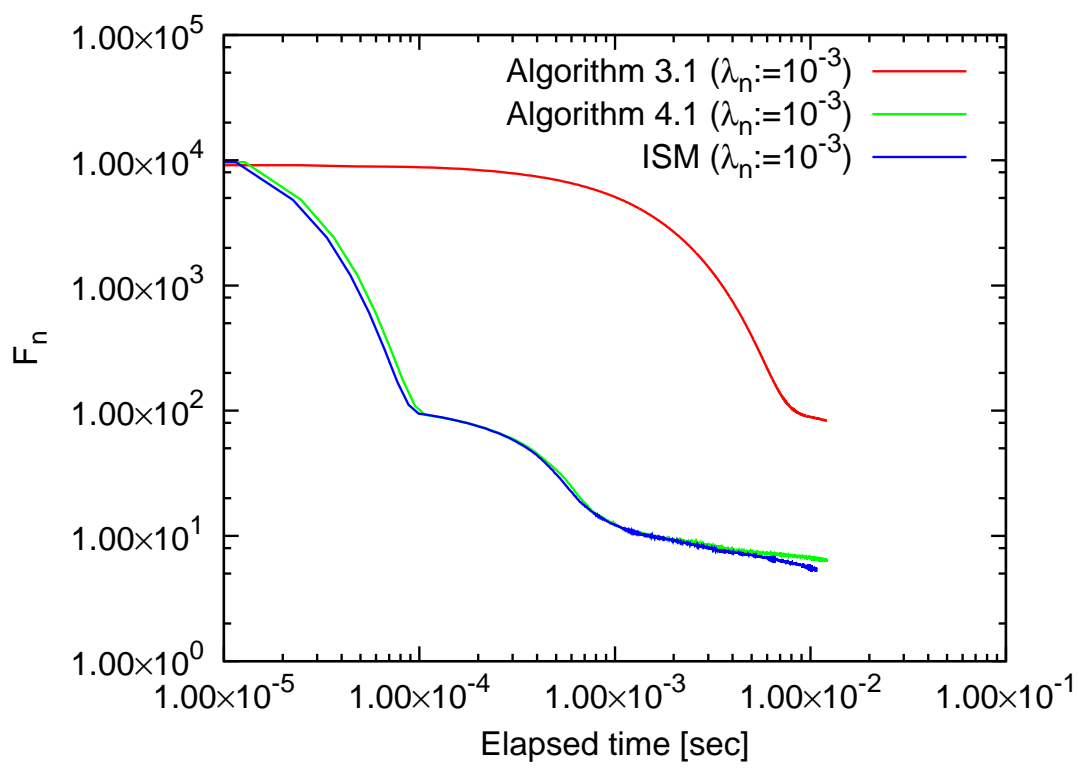

(b) $F_{n}$ vs. elapsed time

Figure 2: Behavior of $F_{n}$ for ISM, Algorithm 3.1, and Algorithm 4.1 when $\lambda_{n}:=10^{-3}$ and $I:=64$ 


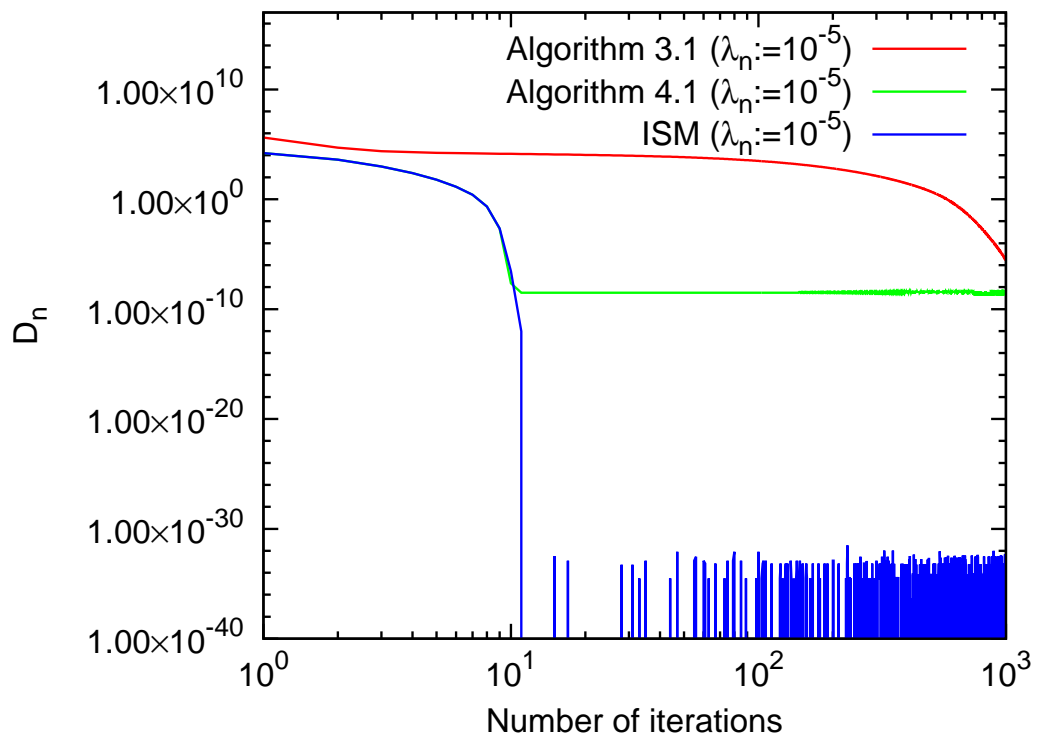

(a) $D_{n}$ vs. no. of iterations

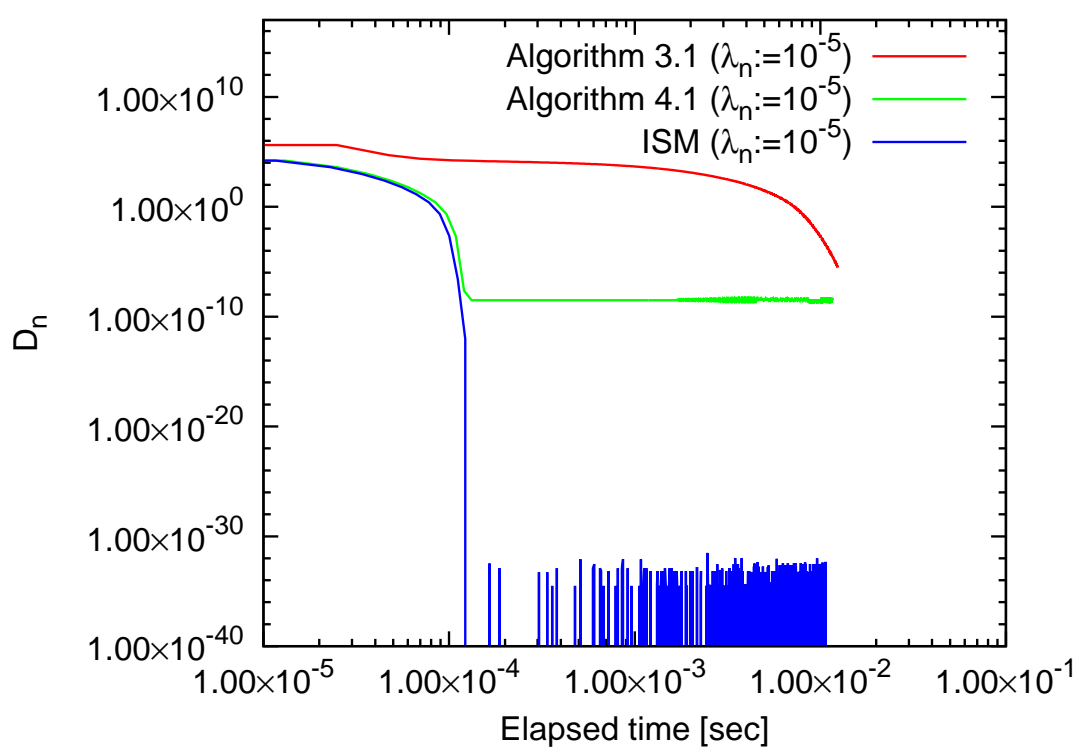

(b) $D_{n}$ vs. elapsed time

Figure 3: Behavior of $D_{n}$ for ISM, Algorithm 3.1, and Algorithm 4.1 when $\lambda_{n}:=10^{-5}$ and $I:=64$ 


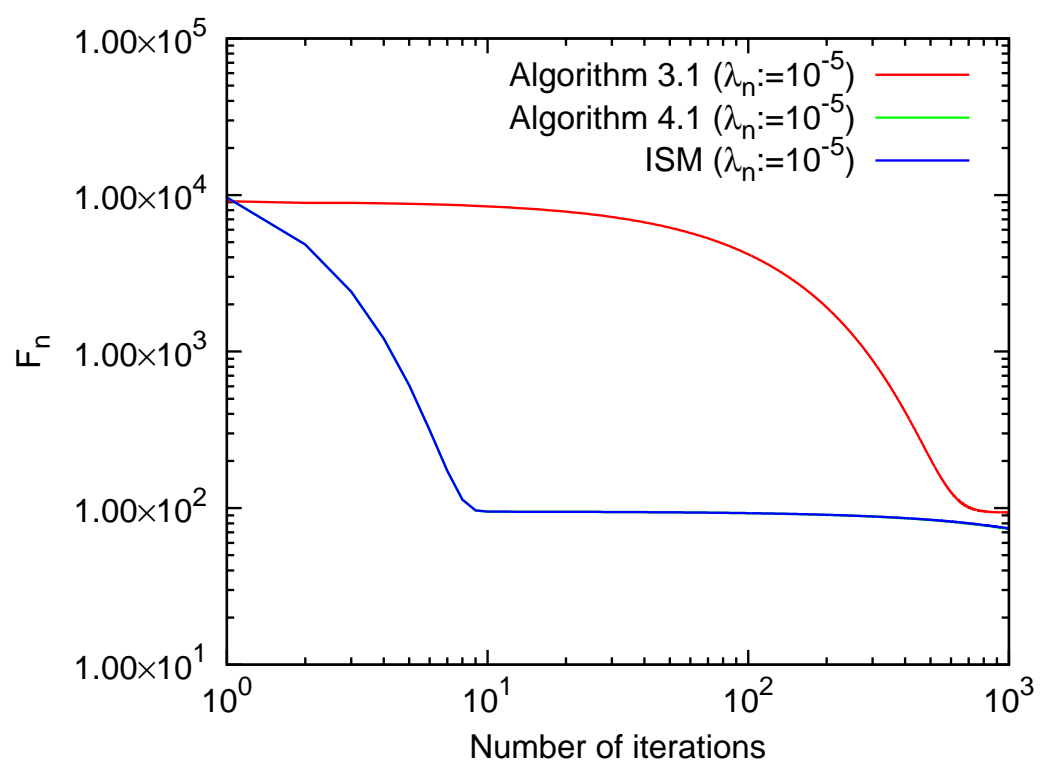

(a) $F_{n}$ vs. no. of iterations

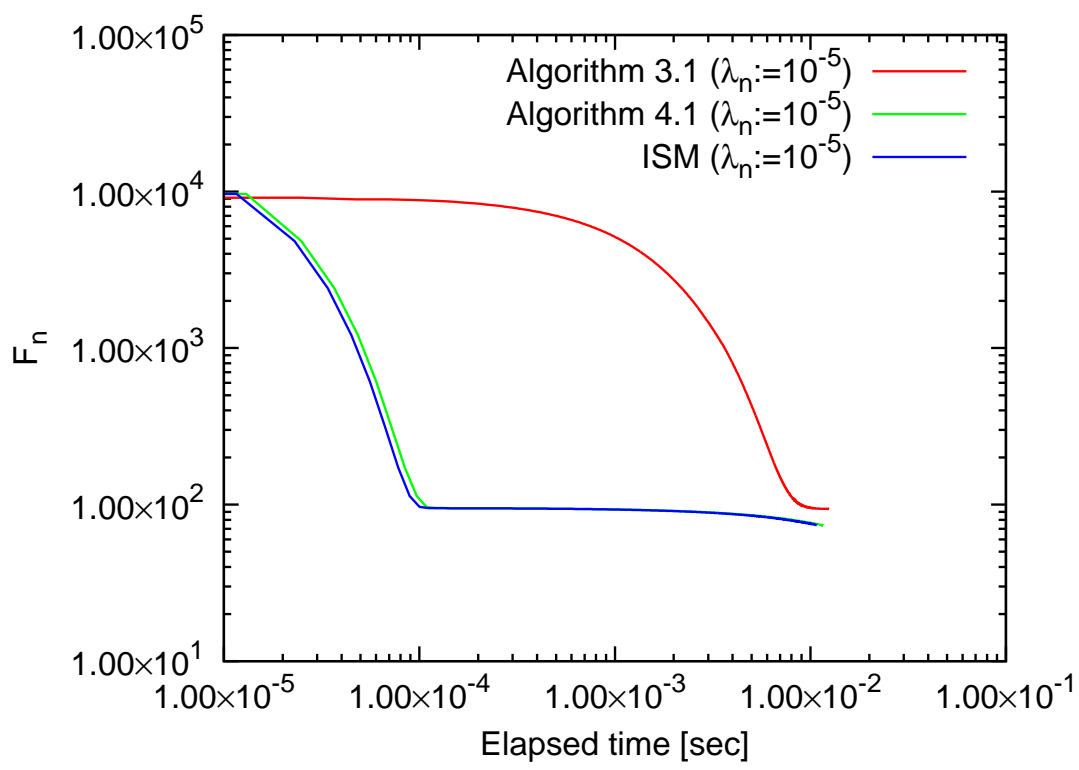

(b) $F_{n}$ vs. elapsed time

Figure 4: Behavior of $F_{n}$ for ISM, Algorithm 3.1, and Algorithm 4.1 when $\lambda_{n}:=10^{-5}$ and $I:=64$ 


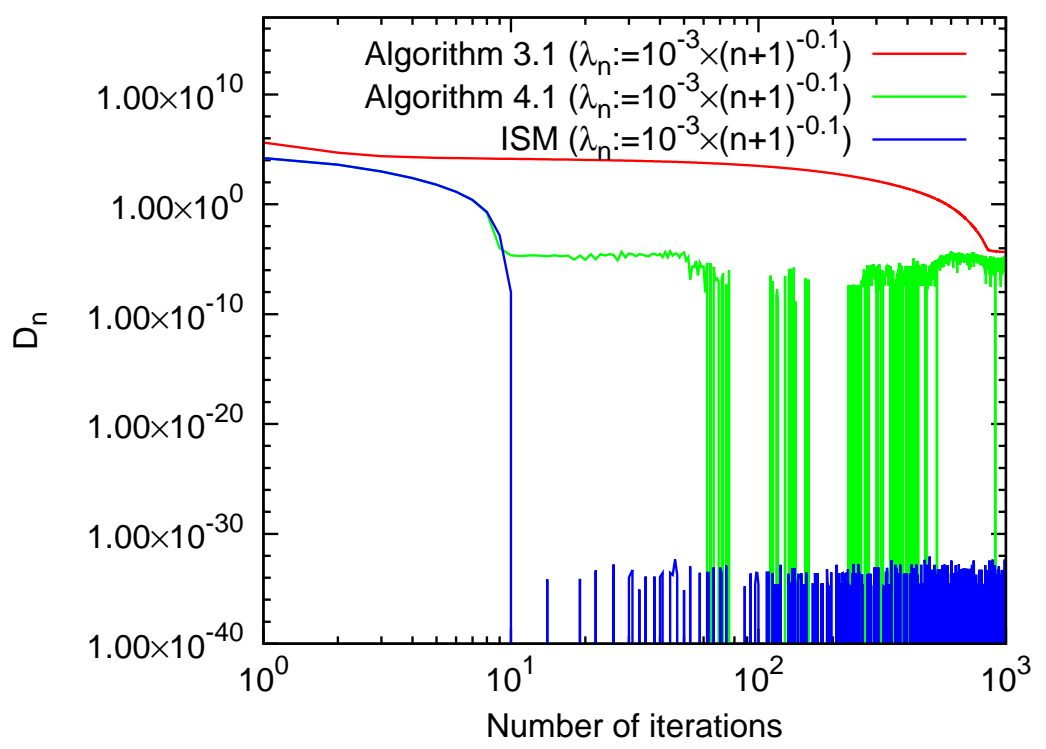

(a) $D_{n}$ vs. no. of iterations

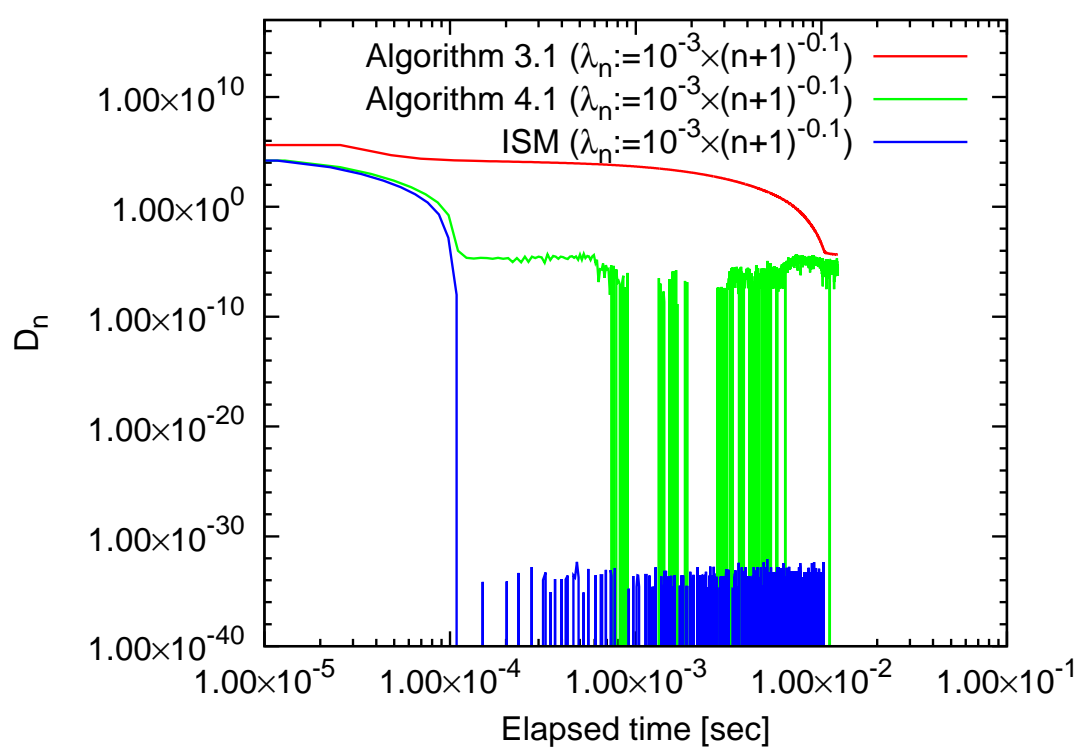

(b) $D_{n}$ vs. elapsed time

Figure 5: Behavior of $D_{n}$ for ISM, Algorithm 3.1, and Algorithm 4.1 when $\lambda_{n}:=10^{-3} /(n+1)^{0.1}$ and $I:=64$ 


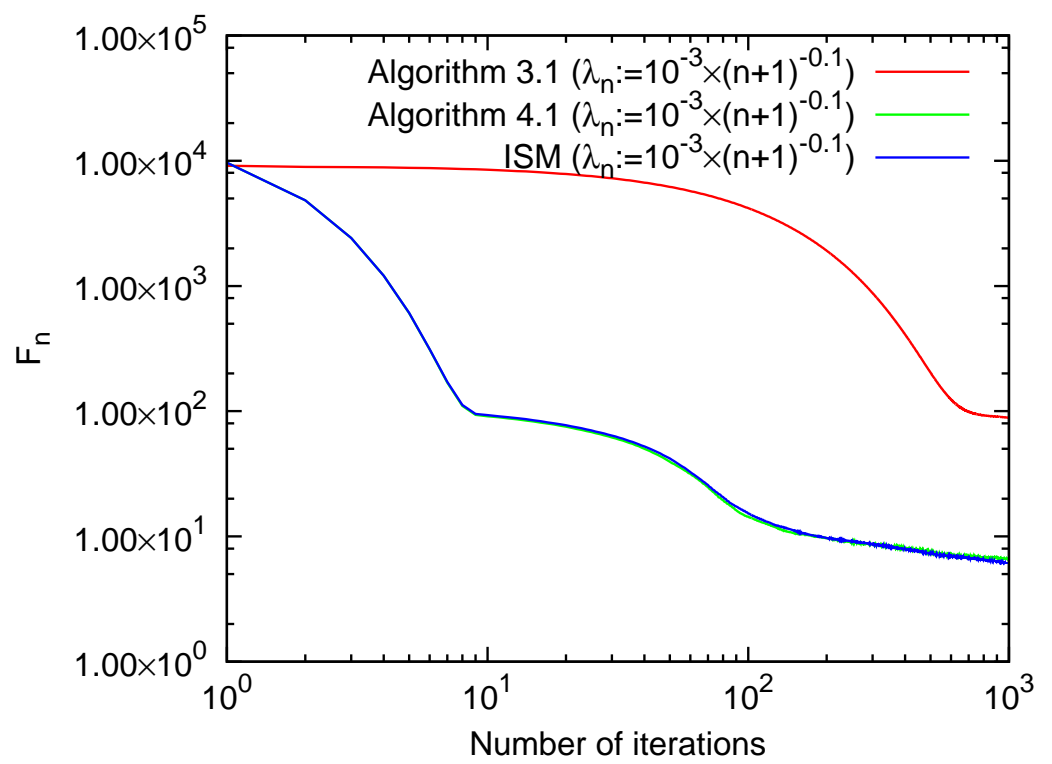

(a) $F_{n}$ vs. no. of iterations

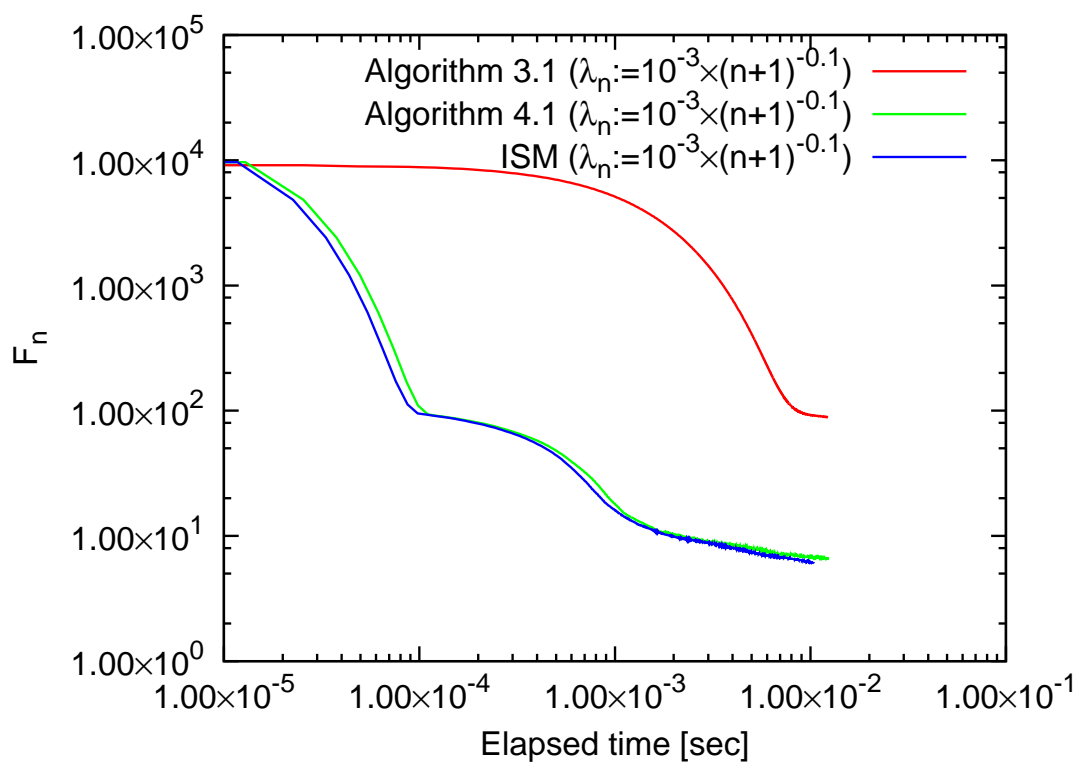

(b) $F_{n}$ vs. elapsed time

Figure 6: Behavior of $F_{n}$ for ISM, Algorithm 3.1, and Algorithm 4.1 when $\lambda_{n}:=10^{-3} /(n+1)^{0.1}$ and $I:=64$ 


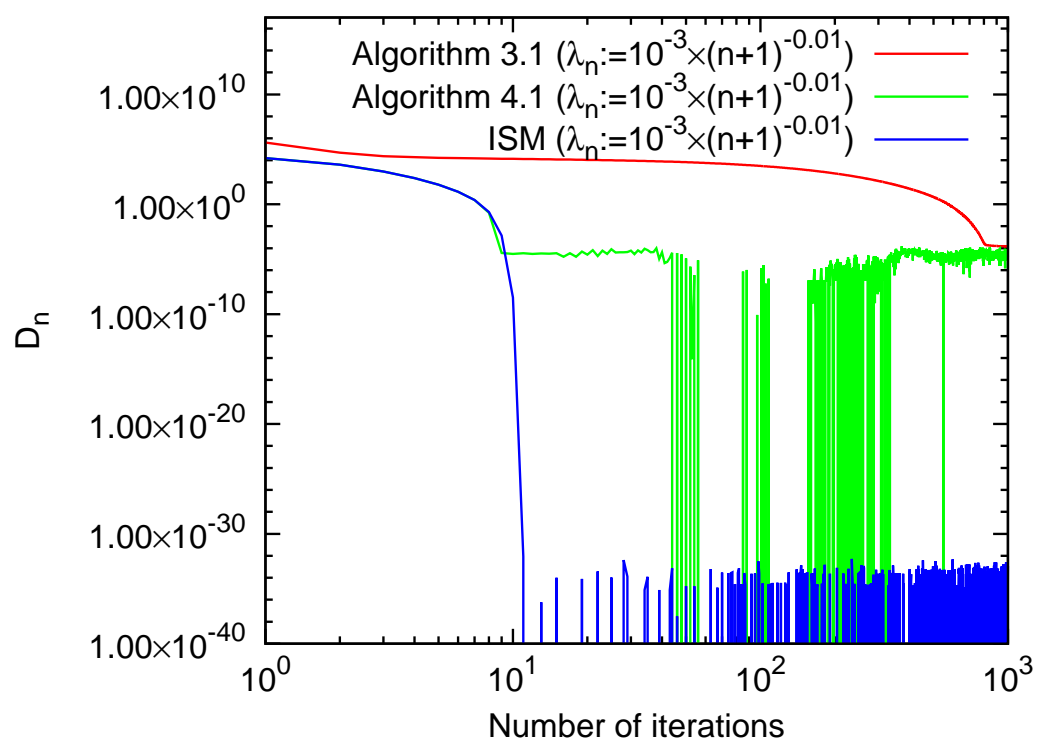

(a) $D_{n}$ vs. no. of iterations

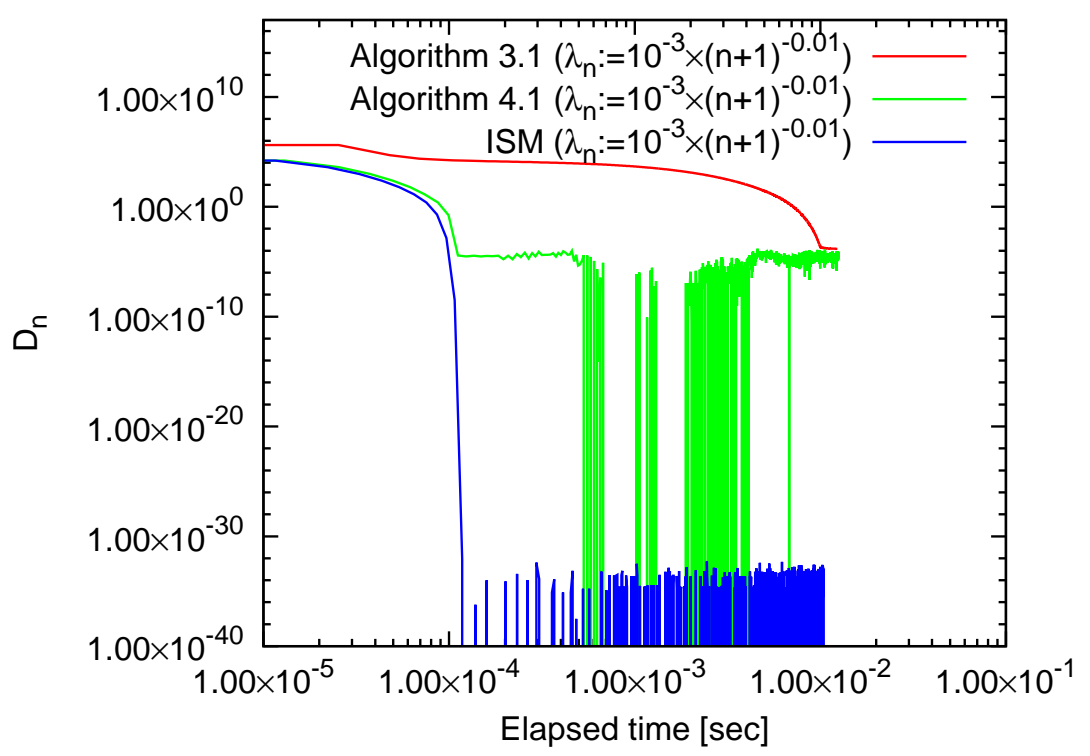

(b) $D_{n}$ vs. elapsed time

Figure 7: Behavior of $D_{n}$ for ISM, Algorithm 3.1, and Algorithm 4.1 when $\lambda_{n}:=10^{-3} /(n+1)^{0.01}$ and $I:=64$ 


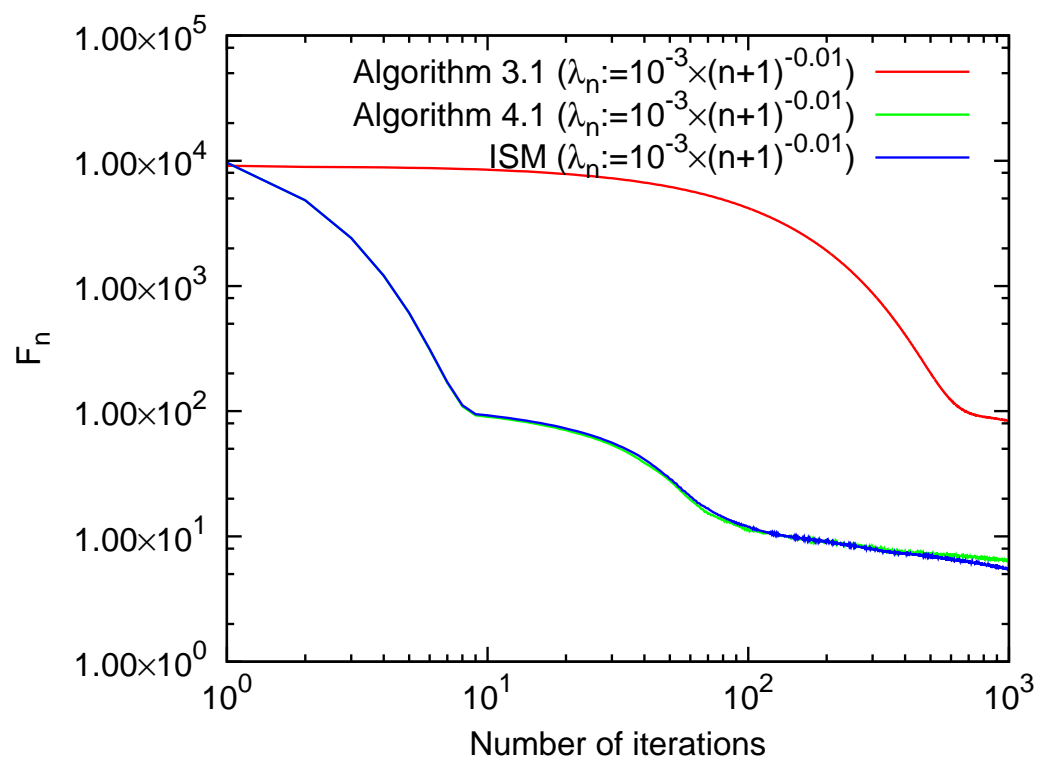

(a) $F_{n}$ vs. no. of iterations

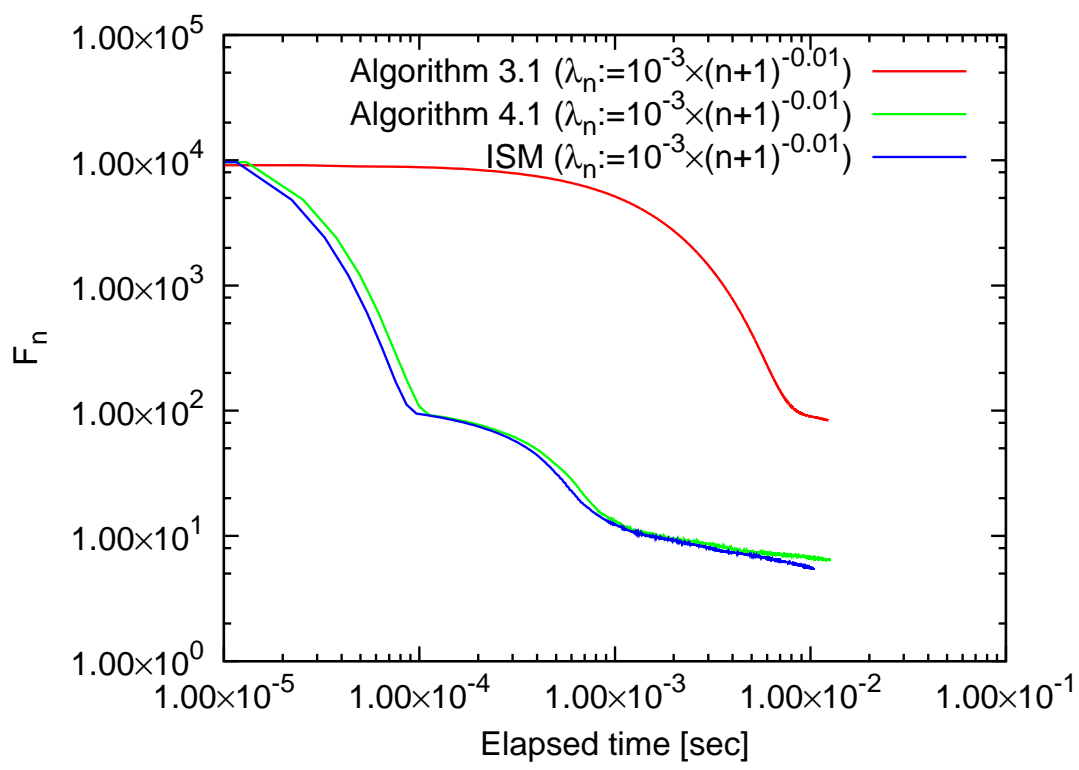

(b) $F_{n}$ vs. elapsed time

Figure 8: Behavior of $F_{n}$ for ISM, Algorithm 3.1, and Algorithm 4.1 when $\lambda_{n}:=10^{-3} /(n+1)^{0.01}$ and $I:=64$ 


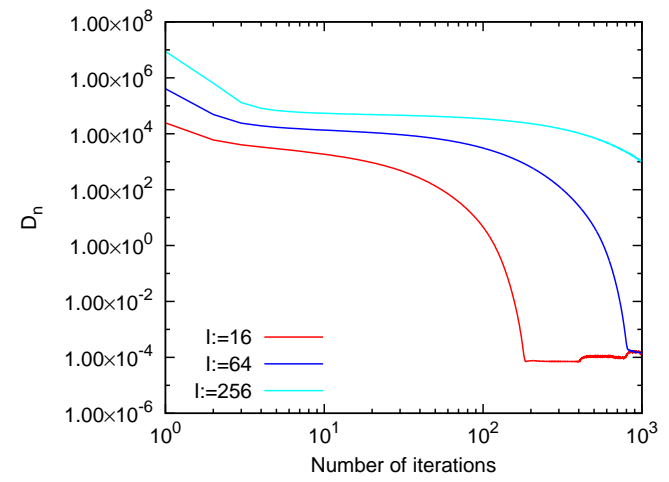

(a) $D_{n}$ vs. no. of iterations

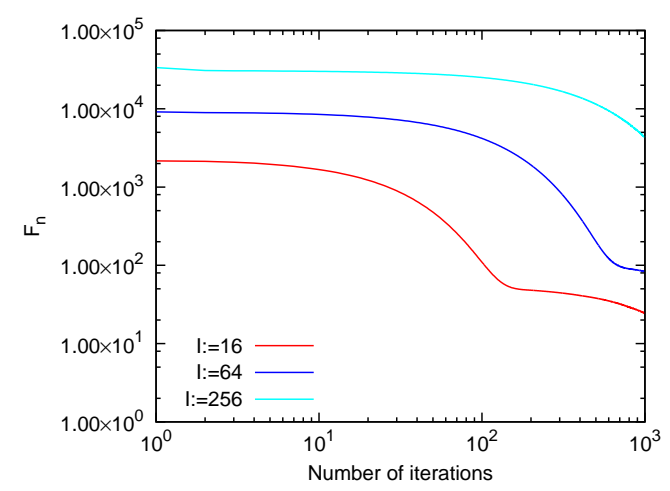

(c) $F_{n}$ vs. no. of iterations

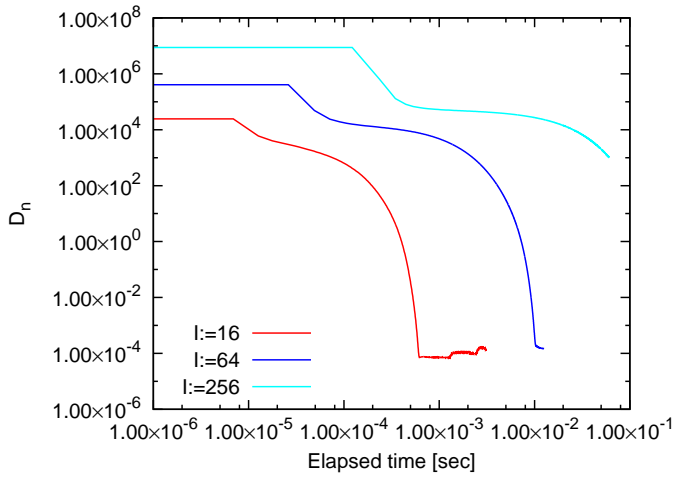

(b) $D_{n}$ vs. elapsed time

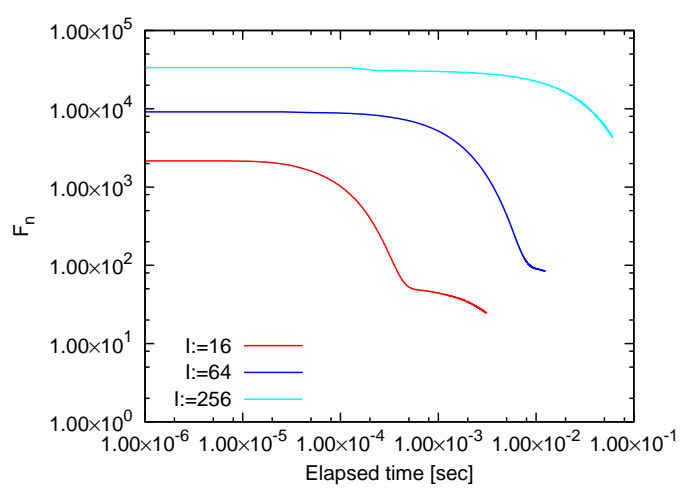

(d) $F_{n}$ vs. elapsed time

Figure 9: Efficiency of Algorithm 3.1 for $I$ when $\lambda_{n}:=10^{-3} /(n+1)^{0.01}$ 


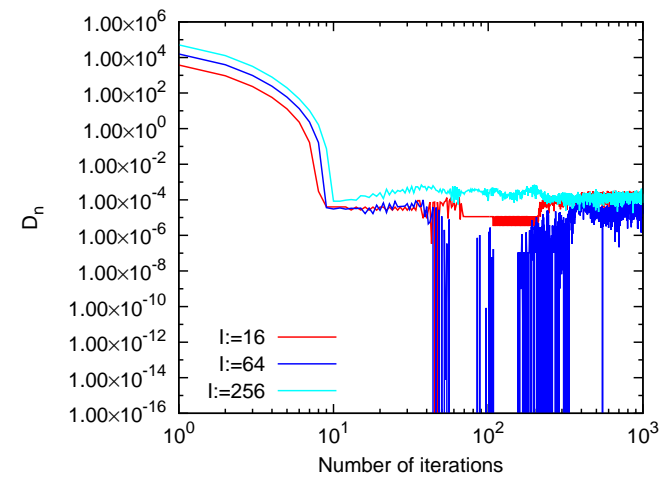

(a) $D_{n}$ vs. no. of iterations

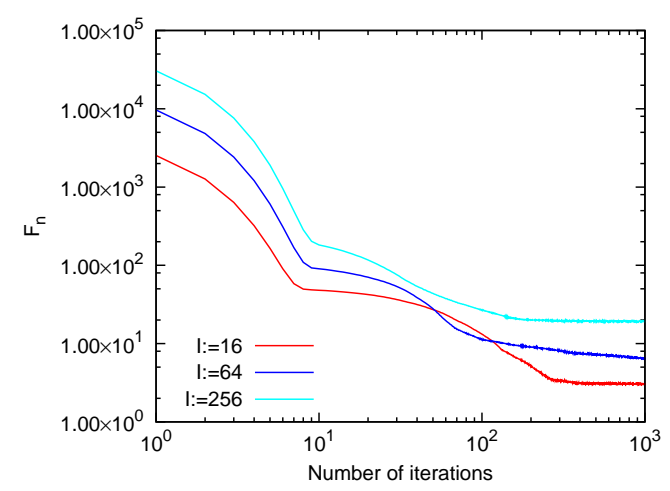

(c) $F_{n}$ vs. no. of iterations

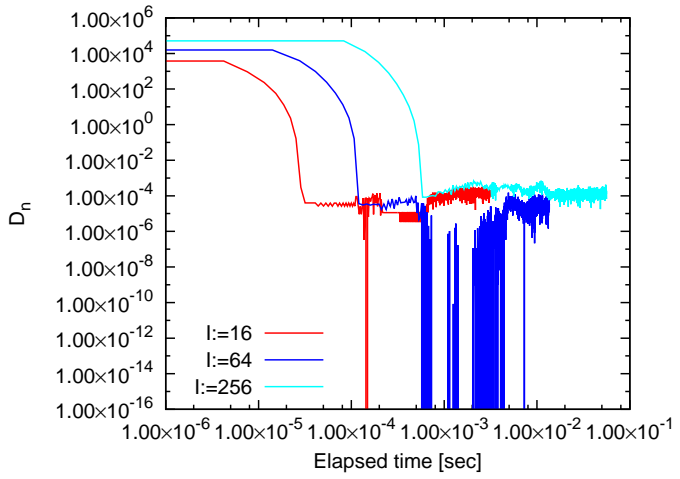

(b) $D_{n}$ vs. elapsed time

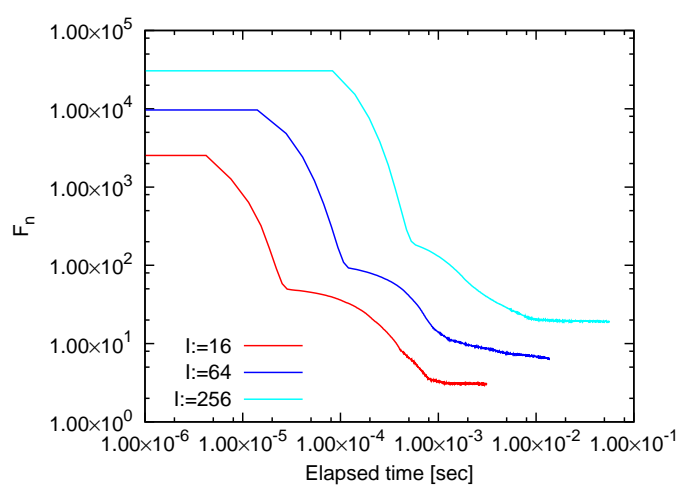

(d) $F_{n}$ vs. elapsed time

Figure 10: Efficiency of Algorithm 4.1 for $I$ when $\lambda_{n}:=10^{-3} /(n+1)^{0.01}$ 


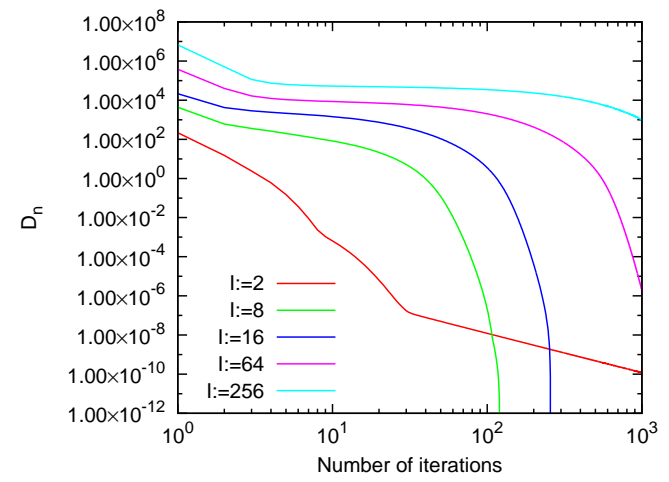

(a) $D_{n}$ vs. no. of iterations

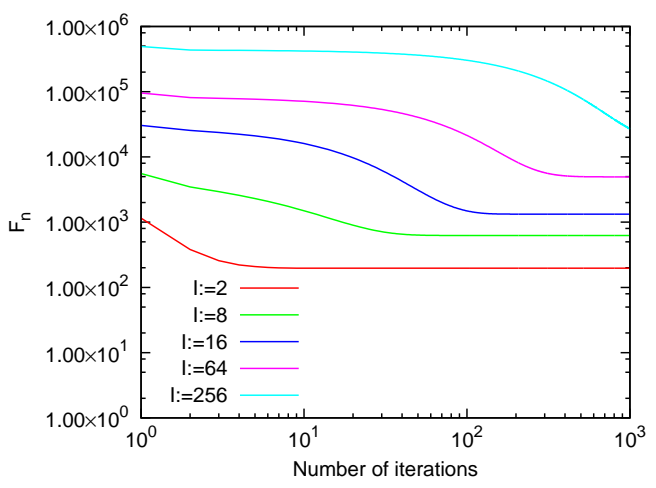

(c) $F_{n}$ vs. no. of iterations

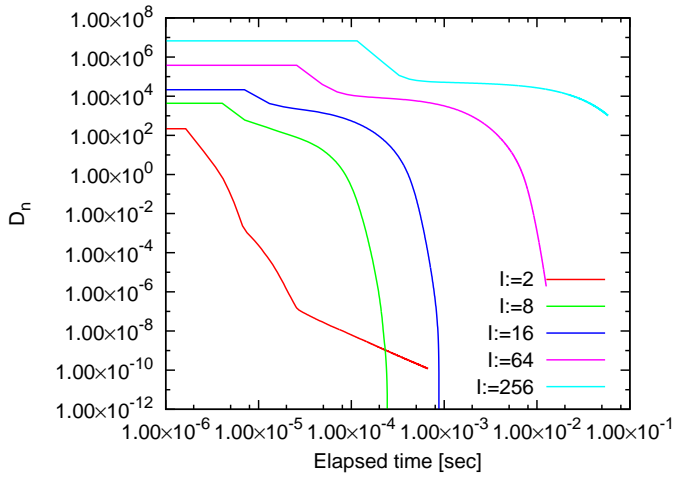

(b) $D_{n}$ vs. elapsed time

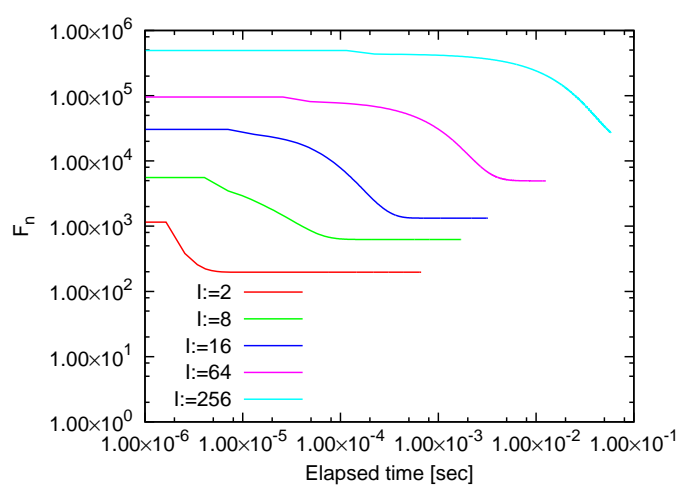

(d) $F_{n}$ vs. elapsed time

Figure 11: Efficiency of Algorithm 3.1 for $I$ when only $f^{(1)}$ is strongly convex and $\lambda_{n}:=10^{-3} /(n+1)$ 


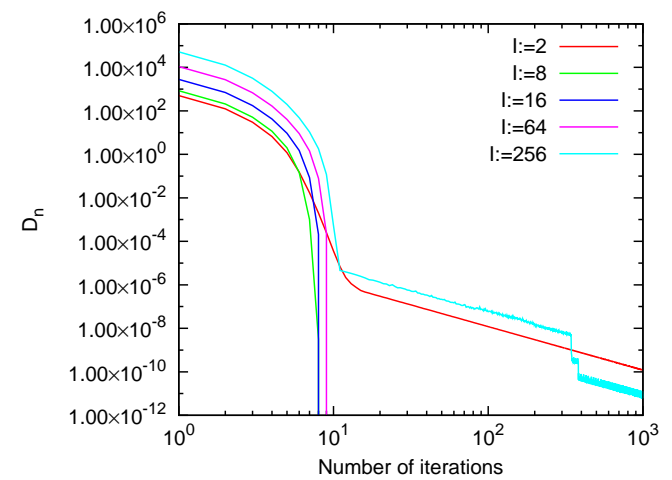

(a) $D_{n}$ vs. no. of iterations

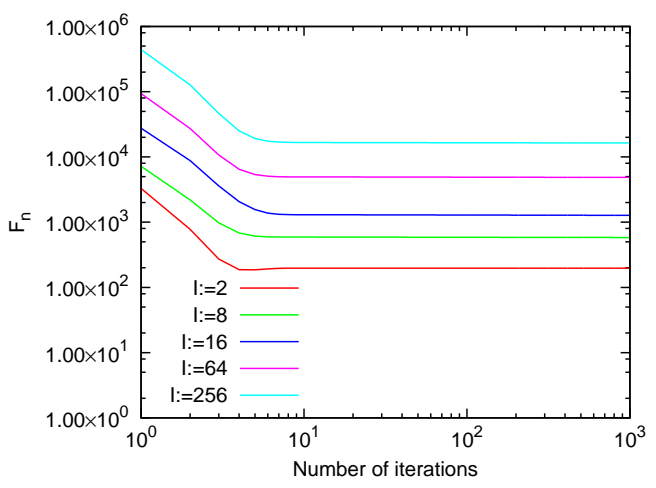

(c) $F_{n}$ vs. no. of iterations

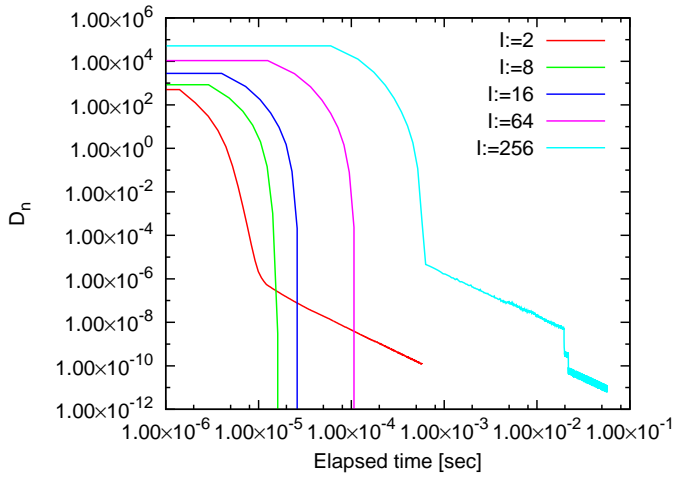

(b) $D_{n}$ vs. elapsed time

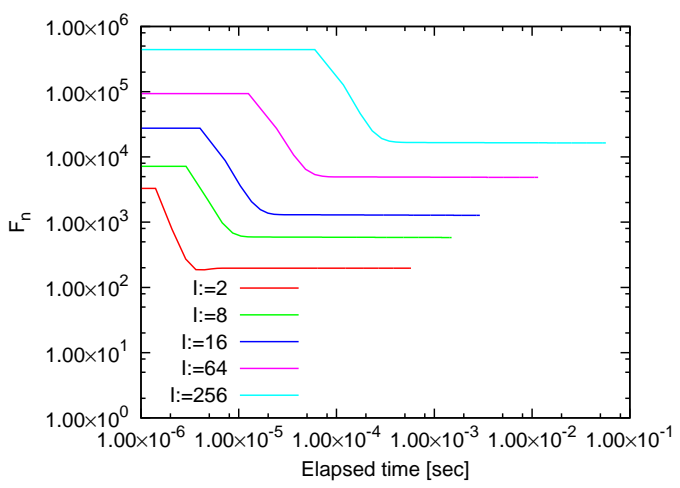

(d) $F_{n}$ vs. elapsed time

Figure 12: Efficiency of Algorithm 4.1 for $I$ when only $f^{(1)}$ is strongly convex and $\lambda_{n}:=10^{-3} /(n+1)$ 